Science

October 2021, Volume 374 Issue 6566 Pages 488-492

https://doi.org/10.1126/science.abb1083

https://archimer.ifremer.fr/doc/00732/84375/

\title{
Rapid evolutionary turnover of mobile genetic elements drives bacterial resistance to phages
}

\author{
Hussain Fatima Aysha 1, Dubert Javier 1, 2, Elsherbini Joseph 1, Murphy Mikayla ${ }^{1}$, \\ Vaninsberghe David 1, Arevalo Philip 1, Kauffman Kathryn 1, Rodino-Janeiro Bruno Kotska 1,3, \\ Gavin Hannah 1, Gomez Annika 1, Lopatina Anna ${ }^{3}$, Le Roux Frederique 4, 5, ${ }^{*}$, Polz Martin F. 1, 3, *
}

${ }^{1}$ Department of Civil and Environmental Engineering, Massachusetts Institute of Technology, Cambridge, MA, USA.

${ }^{2}$ Department of Microbiology and Parasitology, University of Santiago de Compostela, Santiago de Compostela, Spain.

${ }^{3}$ Division of Microbial Ecology, Department of Microbiology and Ecosystem Science, Center for Microbiology and Environmental Systems Science, University of Vienna, Vienna, Austria.

4 Ifremer, Unité Physiologie Fonctionnelle des Organismes Marins, CS 10070, F-29280 Plouzané, France.

${ }^{5}$ Sorbonne Universités, UPMC Paris 06, CNRS, UMR 8227, Integrative Biology of Marine Models, Station Biologique de Roscoff, CS 90074, F-29688 Roscoff Cedex, France.

* Corresponding authors : Frédérique Le Roux, email address : fleroux@sb-roscoff.fr ; Martin F. Polz, email address : martin.f.polz@univie.ac.at

\begin{abstract}
:
Although it is generally accepted that phages drive bacterial evolution, how these dynamics play out in the wild remains poorly understood. We found that susceptibility to viral killing in marine Vibrio is mediated by large and highly diverse mobile genetic elements. These phage defense elements display exceedingly fast evolutionary turnover, resulting in differential phage susceptibility among clonal bacterial strains while phage receptors remain invariant. Protection is cumulative, and a single bacterial genome can harbor 6 to 12 defense elements, accounting for more than $90 \%$ of the flexible genome among close relatives. The rapid turnover of these elements decouples phage resistance from other genomic features. Thus, resistance to phages in the wild follows evolutionary trajectories alternative to those predicted from laboratory-based evolutionary experiments.
\end{abstract}




\section{Main Text:}

Bacterial viruses (phages) are ubiquitous across Earth's biosphere and control microbial populations through predatory interactions $(1-3)$. Because successful killing depends on molecular interaction with the host, phages display higher specificity than most other microbial predators. This is an important reason for renewed interest in the clinical use of phages as alternatives to antibiotics (4). Like antibiotics, however, phage killing exerts strong selection for resistance in bacterial hosts (5). Consequently, how bacteria naturally acquire resistance has important implications for understanding microbial community dynamics as well as the longterm success of phage therapy. Laboratory co-evolution studies have consistently identified phage-receptor mutations as key drivers of resistance $(6,7)$, with genetic analyses indicating secondary contributions by restriction-modification (RM) (8-10) and abortive infection (Abi) systems (11-13). However, because phages target important surface structures, such as the lipopolysaccharide (LPS) and membrane proteins as receptors, it is questionable whether mutations in receptors represent primary adaptive strategies in complex microbial communities since such modifications frequently incur significant fitness costs (6). Indeed, many additional defense mechanisms have recently been discovered, including clustered regularly interspaced short palindromic repeats (CRISPR) systems (14), and several other innate immunity mechanisms, many of which remain to be mechanistically characterized (15-19). Importantly, genes encoding both receptors and defense systems have been shown to occur frequently in variable genomic islands $(6,20-22)$, or associated with mobile genetic elements (23-26). However, while for CRISPR-based adaptive immunity, which is present in over $40 \%$ of bacteria (27), evolutionary processes have been documented on ecological timescales $(28,29)$, much less is known for innate immunity mechanisms, which defend most bacteria in the wild (30). Neither 
the predominant mechanisms of resistance to phages nor the dynamics of resistance gain and loss are well understood $(31,32)$, limiting our knowledge of how phage predation structures diversity and drives evolution of wild microbial populations.

Here we combine population genomic and molecular genetic approaches to determine how phage resistance arises in natural microbial populations lacking adaptive immunity. We recently created a large genomically-resolved phage-host cross-infection network, using sympatric environmental isolates (33-35). The Nahant Collection was established in the context of a 93-consecutive-day coastal-ocean time series (36) and comprises over 1,300 strains of marine Vibrio ranging in relatedness from near-clonal to species-level divergence $(34,37)$. Vibrio hosts isolated on three different days (ordinal dates 222, 261, 286) were used as "bait" to isolate 248 co-occurring lytic viruses by quantitative plaque assays, and each of 245 plaque positive hosts were subsequently challenged with all phage isolates to establish an all-by-all cross-infection matrix (Fig. S1A). Phages tend to be highly specific, as indicated by the general sparsity of the matrix. Additionally, even the most closely related hosts, differentiated by a few single nucleotide polymorphisms (SNPs) across the core genome, are preyed upon by different phages (Fig. S1B). Furthermore, this extends to broader host-range phages that, although capable of infecting multiple hosts, are typically limited to a single strain within a host clade (Fig. S1C).

Because the observation that nearly clonal bacterial strains are subject to differential predation suggests extremely rapid evolution of phage resistance, we sought to identify the mechanisms in an exemplary set of 22, nearly clonal isolates of Vibrio lentus (Fig. 1A, left) that are differentially infected by two highly divergent groups of lytic siphovirus phages consisting of four and 18 isolates, respectively ("orange" and "purple" in Fig. 1A, top; Fig. S2A-D, Data S1). Testing all pairwise interactions of these phages with all potential hosts at varying multiplicity of 
infection (MOI), reveals two phenotypes: (i) host lysis and phage production and (ii) host lysis 80 only at high phage concentrations without phage production (Fig. S3). The latter effect, termed "lysis from without" (38), along with observed adsorption of representative phages to differing hosts (Fig. S4), suggests that the phages can adsorb to a broader set of hosts than they can kill.

Supporting our hypothesis, we find that while the two phage types use different receptors, all bacterial genomes encode both sets of receptors. Molecular genetic analysis suggests the "orange" phage receptor is the Type II secretion system (T2SS) pseudopilus (GspH), while the "purple" phages have two receptors, one being LPS, an extracellular receptor, and the second being a sodium transporter (NqrC), a membrane receptor ((39), Fig. S5, Tables S1 and S2). Comparative genomics showed that every identified gene encoding a phage receptor is identical across all 23 "orange", "purple" and outgroup strains. The noteworthy exception is the single outgroup strain (10N26154A12) that differs by one SNP in $g s p N$ : N201K, which prevents a single "orange" phage 1.127.0 from attaching (Fig. 1A). Although this suggests receptor mutations play a secondary role, the data overall support that receptors are not primarily responsible for the observed phage specificity, leading us to explore potential intracellular mechanisms of host resistance by a combination of comparative genomics and molecular 95 genetics.

Clustering the pangenome of the 22 near clonal Vibrio lentus isolates using high-quality genomes, together with a slightly more divergent outgroup for additional genomic context, (Fig. 1B, right) reveals the presence-absence patterns of large flexible genomic regions containing putative phage defense genes. Using a k-mer-based approach to conduct all-by-all pair-wise genome comparisons (Data S2), we identified a total of 28 flexible genome regions ( $>5 \mathrm{~kb}$ in length), 26 of which show clear hallmarks of being mobile genetic elements (MGEs) and 
harboring at least one gene related to a known phage defense gene (Data S3). These elements range in size from 8,500 to 89,600 bp and contain genes annotated as recombinases/integrases and transposases (Data S3), but the elements appear overall unrelated since only few genes are 105 shared (Fig. 1C, Data S3). Except for two episomal elements, one of which is a prophage, all others are chromosomally integrated and their presence-absence patterns in different clonal genomes indicate that these regions are transferred as independent units (Fig. S6). Of the chromosomally integrated elements, three are also putative prophages. We refer to these 26 MGEs as phage defense elements (PDEs) since no other function obviously relevant to the host 110 cell can be identified. Their number ranges between six and 12 in each strain, and collectively, the PDEs account for over $95 \%$ of the flexible genomic regions (Fig. 1D, Data S3). Even if only known defense genes, excluding the entire PDEs are considered, phage defense genes account for $>20 \%$ of the flexible gene content (Fig. 1D). We observe a similar range of $12-17 \%$ in diverse Vibrio species in our collection (Fig. S7) even though these comprise higher genomic

115 diversity than the clonal strains and have known flexible genome elements other than those involved in phage defense (40). If we consider that most of the remaining PDE genes may be involved in maintenance and spread, and that we did not detect any other functional annotations on the PDEs, we predict a large, if not the largest, portion of the flexible genome might be involved in phage defense.

To investigate the impact of PDEs on phage defense, we looked more closely at a near clonal subset of these hosts that have differential presence of PDEs matched by phage predation profiles. These hosts consist of a group of four strains and another group of 15 strains that are only differentiated by 14 SNPs across the core genome, yet are differentially infected by the two phage groups (Fig. 1A, Fig. S8, Table S3). The flexible genome of these "orange" and "purple" 
hosts is differentiated by three (PDEs 1-3) and two (PDEs 4-5) large PDEs (Fig. 1E). PDE1, PDE4, and PDE5 have Type 1 RM systems, and PDE2 and PDE3 have several putative defense genes. These include many genes related to abortive infection (Abi) systems, as well as genes with domains homologous to retrons, reverse transcriptases, and GTPases $(17,22)$. A striking feature of the PDEs is that their insertion does not appear to disrupt host functions. For example, PDE1 inserts into "orange" hosts' 5'-deoxynucleotidase nucleosidase $(y f b r)$ gene, thereby truncating it, but encodes its own divergent copy of the same gene. Similarly, although PDE2 disrupts a gene encoding a thiol peroxidase upon insertion, it encodes a copy in the middle of the element (Fig. 1E). These five PDEs are also found in more divergent Vibrio genomes, which indicates they transfer via horizontal gene transfer (Fig. S9), sometimes inserting into different genomic neighborhoods, although at conserved insertion motifs with the exception of a few SNPs (Fig. S10).

We asked to what extent do each of the PDEs contribute to the differential resistance observed between the "purple" and "orange" hosts? All three PDE-encoded RM systems (PDEs1,4 and 5; Fig. 1E) appear active based on methylome sequencing analysis (Table S4, 140 (39)). To further characterize the contribution to resistance of other potential defense genes, we focused on the three PDEs specific to "orange" host strains as an exemplar. First, we found that all three PDEs are transcribed during an infection with a "purple" phage (Fig. S11). Second, we systematically knocked out large regions of the three PDEs containing putative defense as well as unidentified candidate genes hypothesized to drive resistance of a representative "orange" host 145 strain (10N26155C8) to "purple" phages (Fig. 2A), and then challenged the knockouts with all "purple" and "orange" phages (Fig. 2B, Fig. S12; Fig. S13). This genetic analysis shows that in addition to the RM-encoding PDE, both PDEs encoding alternative defense systems are also 
active and together contribute to phage resistance since deletion of all three PDEs is required for full sensitivity.

The complexity of the interaction among all PDEs leading to full resistance is illustrated by deletion experiments covering all possible combinations. Knocking out RM-containing PDE1 alone increased the "lysis from without" phenotype tenfold (from $10^{-1}$ to $10^{-2}$ phage dilution), but did not yield viable phage progeny when assayed using a re-streaking test (Fig. 2C). This phenotype led us to hypothesize that there may be a multi-level defense structure involving the remaining two PDEs. Knocking out PDE2 and PDE3 independently resulted in no change from the wild type (WT) phenotype. However, knocking out PDE2 and PDE3 together allowed for both killing and propagation of the phage at a wider range of high MOIs ( $\leq 10^{-5}$ phage dilution). This phenotype is consistent with restriction modification escape mutants ((39), Fig. S14). Knocking out PDE1 and PDE2 together resulted in the same tenfold increase in killing observed 160 when deleting PDE1 alone, while knocking out PDE1 and PDE3 together yields cell death at much lower MOIs ( $10^{-5}$ phage dilution), but importantly, in neither case were viable phages propagated (Fig. 2C). These data suggest that both PDEs afford protection but that PDE3 provides stronger resistance than PDE2. Finally, knocking out all three PDEs simultaneously resulted in the "orange" strain becoming just as susceptible to the "purple" phages as are 165 "purple" WT host strains. Therefore, we conclude all three elements are needed for full, WTlevel defense.

Further genetic characterization of defense genes within the PDEs underscores the diversity of undiscovered or poorly characterized genes with anti-phage function. We took advantage of our double PDE mutants to scrutinize the remaining active PDE for phage defense. While we were only able to find a single gene (the restriction enzyme) necessary for defense on 
PDE1 (Figs. 2A, S15), three unannotated genes and two putative GTPases within a module on the 5'end of PDE2 appear involved in defense (Figs. 2A, S16). For PDE3, we inactivated 11 different genes as well as two regions associated the two retrons present; however, none showed a loss of resistance phenotype. We therefore analyzed the host transcriptome during infection with a "purple" phage and identified 14 highly expressed and/or co-transcribed regions (Fig. S17A). Using the triple knockout mutant, $\Delta \Delta \Delta \mathrm{PDE} 123$, we complemented eight of these regions with their native promoter and observed restoration of phage defense by region R3 containing a gene with DUF2971 (Figs. 2A, S17B,C). Hence two of the three PDEs carry uncharacterized phage defense genes.

That receptor genes are invariant across the two near clonal host groups challenges the notion that receptor variation is primarily responsible for resistance $(6,20)$. Thus, we asked to what extent these receptors are variable across more diverse populations. Populations are defined here as gene flow clusters that also represent ecological units $(37,41)$. The recognition of population boundaries is key for interpretation of gene or allele frequency in the context of 185 differential selective forces. Surprisingly, looking across 107 Vibrio isolates, spanning 10 populations, all putative receptors are highly monomorphic at the population level indicating they are under purifying selection. The two genes identified as receptors in $V$. lentus are identical, or nearly so, at the amino acid sequence level across populations and thus well below the average diversity of core genes (Fig. S18A). This is corroborated by phylogenetic trees based 190 on nucleotide sequences showing that all members of each population carry the same or highly similar gene variants, a pattern consistent with the occurrence of recent gene-specific selective sweeps. A notable exception is $g s p H$ which is more diverse in two of the populations (Fig. S18B). Finally, the LPS, which frequently serves as the primary receptor for many phages, also 
appears similar at the population level since the genes responsible for its synthesis display population-specific presence/absence patterns suggesting the synthesis pathway is conserved (Fig. S18C). Thus, although putative receptors can reside in variable regions when more divergent genomes are compared (20), their evolution appears to be constrained when population structure is considered. This constraint may arise because in wild populations, these surface structures are optimized for ecological interactions, and indicates a key difference from the lab where, in rich media, receptor mutations frequently arise in phage-host co-cultures (42). This observed invariance suggests that other selective forces that compete with phage resistance play an important role in receptor evolution in the wild. This interpretation is consistent with predictions that intracellular defenses should be important under such conditions $(43,44)$, as well as with phage-host evolution in nature differing from the lab (45). It is possible that receptormediated defenses may only be advantageous under extreme predation regimes, or under regimes of low effective diversity, such as a clonal infection or laboratory culture.

Defense being largely determined by differential PDE carriage at the clonal level suggests that the rate of turnover is surprisingly fast and confirms theoretical considerations that resistance genes should be mobile because the cost of resistance limits their utility under changing predation pressures (25). Genomic comparisons of the 23 strains illustrates this high turnover since genomes with zero SNPs can differ by at least one putative PDE, and the "purple" and "orange" hosts groups carry a minimum difference of five PDEs while being separated by only 14 SNPs (Fig. 1 and Fig. S8). Further, across the time series of a mere 90 days, we observe a differential shift in relative PDE abundance. We designed droplet digital PCR assays to span 215 the insertion sites of three of the PDEs to constrain the detection of the resistance elements to closely related genetic backgrounds (Fig. S18). While PDE3 and PDE4, representing “orange” 
and "purple" genetic backgrounds, respectively, are near perfectly correlated across the entire time series, PDE2 only gains in importance towards the end of the time series. These dynamics suggest rapid evolution at the population level, either from acquisition of PDE2 or a shift in abundance of host clones with differential PDE carriage. Both scenarios reflect a rapidly shifting selective landscape of host-phage interactions.

PDEs structuring host defense among near clonal isolates is likely general beyond the detailed description provided here. Within other Vibrio species in our collection, clonal isolates also differ in their phage predation profiles (Fig. S1), and, even among bacterial pathogens that in contrast to vibrios - follow a primarily non-recombinogenic mode of evolution $(37,46-48)$, we observe similarly rapid turnover of putative phage defense elements, several of which are prophages themselves. When analyzing publicly available genomes with identical ribosomal protein gene sequences from Listeria (Fig. S20, Data S4), Salmonella (Fig. S21, Data S5), and Clostridium (Fig. S22, Data S6), we observe a surprisingly similar dominance $(\sim 90 \%)$ of the flexible genome associated with phage defense regions across these diverse species.

We have shown that innate immunity mechanisms can be rapidly turned over on the population level, with three major implications. First, because co-existing diversity in many natural bacterial populations is high (49), resistance at the clonal level effectively lowers prey concentration and thus phage-host encounter. Second, encoding resistance on highly mobile elements can make phage resistance a fast-changing phenotypic trait independent of physiological or metabolic traits encoded by the core genome. This allows the core genome to be maintained over the long-term even in the face of phage predation. In other words, while phages may exert negative frequency dependent selection on their hosts, this selection is largely acting at the level of MGEs. Finally, rapid acquisition of resistance may also have to be considered for 
repeated or longer-term use of phages in therapy since resistance may be easily acquired and quickly spread through bacterial populations, just as the connection to MGEs (i.e., primarily plasmids) has led to an unanticipated rise in antibiotic resistance. An important open question is therefore what the mechanisms of transfer and life history strategies of PDEs are. Together, our findings suggest that phage resistance is an important, if not the most important, selective force determining clonal bacterial diversity, with phage defense elements potentially explaining a very large portion of the previously enigmatic bacterial flexible genome.

\section{References and Notes:}

1. K. E. Wommack, R. R. Colwell, Virioplankton: Viruses in aquatic ecosystems. Microbiol. Mol. Biol. Rev. 64, 69-114 (2000).

2. M. Breitbart, F. Rohwer, Here a virus, there a virus, everywhere the same virus? Trends Microbiol. 13, 278284 (2005).

3. H. G. Hampton, B. N. J. Watson, P. C. Fineran, The arms race between bacteria and their phage foes. Nature. 577, 327-336 (2020).

4. K. E. Kortright, B. K. Chan, J. L. Koff, P. E. Turner, Phage Therapy: A renewed approach to combat antibioticresistant bacteria. Cell Host Microbe. 25, 219-232 (2019).

5. S. J. Labrie, J. E. Samson, S. Moineau, Bacteriophage resistance mechanisms. Nat. Rev. Microbiol. 8, 317-327 (2010).

6. S. Avrani, O. Wurtzel, I. Sharon, R. Sorek, D. Lindell, Genomic island variability facilitates Prochlorococcusvirus coexistence. Nature. 474, 604-608 (2011).

7. J. R. Meyer, D. T. Dobias, J. S. Weitz, J. E. Barrick, R. T. Quick, R. E. Lenski, Repeatability and contingency in the evolution of a key innovation in phage lambda. Science. 335, 428-432 (2012).

8. W. Arber, Host-controlled modification of nacteriophage. Annu. Rev. Microbiol. 19, 365-378 (1965).

9. G. G. Wilson, N. E. Murray, Restriction and modification systems. Annu. Rev. Genet. 25, 585-627 (1991).

10. G. Bertani, J. J. Weigle, Host controlled variation in bacterial viruses. J. Bacteriol. 65, 113-121 (1953).

11. I. J. Molineux, Host-parasite interactions: recent developments in the genetics of abortive phage infections. New Biol. 3, 230-236 (1991).

12. L. Snyder, Phage-exclusion enzymes: a bonanza of biochemical and cell biology reagents? Mol. Microbiol. 15, 415-420 (1995). 
13. A. Lopatina, N. Tal, R. Sorek, Abortive Infection: Bacterial suicide as an antiviral immune strategy. Annu. Rev. Virol. 7, 371-384 (2020).

14. R. Barrangou, C. Fremaux, H. Deveau, M. Richards, P. Boyaval, S. Moineau, D. A. Romero, P. Horvath, CRISPR provides acquired resistance against viruses in prokaryotes. Science. 315, 1709-1712 (2007).

15. S. Doron, S. Melamed, G. Ofir, A. Leavitt, A. Lopatina, M. Keren, G. Amitai, R. Sorek, Systematic discovery of antiphage defense systems in the microbial pangenome. Science, 359, 6379 (2018).

16. A. Millman, A. Bernheim, A. Stokar-Avihail, T. Fedorenko, M. Voichek, A. Leavitt, Y. Oppenheimer-Shaanan, R. Sorek, Bacterial retrons function in anti-phage defense. Cell. 183, 1551-1561 (2020).

17. L. Gao, H. Altae-Tran, F. Böhning, K. S. Makarova, M. Segel, J. L. Schmid-Burgk, J. Koob, Y. I. Wolf, E. V. Koonin, F. Zhang, Diverse enzymatic activities mediate antiviral immunity in prokaryotes. Science. 369, 1077-1084 (2020).

18. R. P. Novick, G. E. Christie, J. R. Penadés, The phage-related chromosomal islands of Gram-positive bacteria. Nat. Rev. Microbiol. 8, 541-551 (2010).

19. A. A. Govande, B. Duncan-Lowey, J. B. Eaglesham, A. T. Whiteley, P. J. Kranzusch, Molecular basis of CDNTase nucleotide selection in CBASS anti-phage defense. Cell Rep. 35, 109206 (2021).

20. F. Rodriguez-Valera, A.B. Martin-Cuadrado, B. Rodriguez-Brito, L. Pasic, T. F. Thingstad, F. Rohwer, A. Mira, Explaining microbial population genomics through phage predation. Nat. Rev. Microbiol. 7, 828-836 (2009).

21. K. S. Makarova, Y. I. Wolf, S. Snir, E. V. Koonin, Defense islands in bacterial and archaeal genomes and prediction of novel defense systems. J. Bacteriol. 193, 6039-6056 (2011).

22. A. Bernheim, R. Sorek, The pan-immune system of bacteria: antiviral defence as a community resource. Nat. Rev. Microbiol., 1-7 (2019).

23. N. D. McDonald, A. Regmi, D. P. Morreale, J. D. Borowski, E. F. Boyd, CRISPR-Cas systems are present predominantly on mobile genetic elements in Vibrio species. BMC Genomics. 20, 105 (2019).

24. A. C. McKitterick, K. D. Seed, Anti-phage islands force their target phage to directly mediate island excision and spread. Nat. Commun. 9, 2348 (2018).

25. E. V. Koonin, K. S. Makarova, Y. I. Wolf, M. Krupovic, Evolutionary entanglement of mobile genetic elements and host defence systems: guns for hire. Nat. Rev. Genet. 21, 119-131 (2020).

26. K. N. LeGault, S. G. Hays, A. Angermeyer, A. C. McKitterick, F. Johura, M. Sultana, T. Ahmed, M. Alam, K. D. Seed, Temporal shifts in antibiotic resistance elements govern phage-pathogen conflicts. Science. 373, 6554 (2021).

27. K. S. Makarova, D. H. Haft, R. Barrangou, S. J. J. Brouns, E. Charpentier, P. Horvath, S. Moineau, F. J. M. Mojica, Y. I. Wolf, A. F. Yakunin, J. van der Oost, E. V. Koonin, Evolution and classification of the CRISPRCas systems. Nat. Rev. Microbiol. 9, 467-477 (2011).

28. A. F. Andersson, J. F. Banfield, Virus population dynamics and acquired virus resistance in natural microbial communities. Science. 320, 1047-1050 (2008).

29. E. Laanto, V. Hoikkala, J. Ravantti, L.-R. Sundberg, Long-term genomic coevolution of host-parasite interaction in the natural environment. Nat. Commun. 8, 111 (2017). 
30. T. Dimitriu, M. D. Szczelkun, E. R. Westra, Evolutionary ecology and interplay of prokaryotic innate and adaptive immune systems. Curr. Biol. CB. 30, 1189-1202 (2020).

31. A. Chevallereau, B. J. Pons, S. van Houte, E. R. Westra, Interactions between bacterial and phage communities in natural environments. Nat. Rev. Microbiol., 1-14 (2021).

32. S. van Houte, A. Buckling, E. R. Westra, Evolutionary ecology of prokaryotic immune mechanisms. Microbiol. Mol. Biol. Rev. 80, 745-763 (2016).

33. K. M. Kauffman, J. M. Brown, R. S. Sharma, D. VanInsberghe, J. Elsherbini, M. Polz, L. Kelly, Viruses of the Nahant sollection, characterization of 251 marine Vibrionaceae viruses. Sci. Data. 5, 180114 (2018).

34. K. M. Kauffman, F. A. Hussain, J. Yang, P. Arevalo, J. M. Brown, W. K. Chang, D. VanInsberghe, J. Elsherbini, R. S. Sharma, M. B. Cutler, L. Kelly, M. F. Polz, A major lineage of non-tailed dsDNA viruses as unrecognized killers of marine bacteria. Nature. 554, 118-122 (2018).

35. K. M. Kauffman, W. K. Chang, J. M. Brown, F. A. Hussain, J. Y. Yang, M. F. Polz, L. Kelly, Resolving the structure of phage-bacteria interactions in the context of natural diversity. BioRxiv p. 2021.06.27.449121, (2021), doi:10.1101/2021.06.27.449121.

36. A. M. Martin-Platero, B. Cleary, K. Kauffman, S. P. Preheim, D. J. McGillicuddy, E. J. Alm, M. F. Polz, High resolution time series reveals cohesive but short-lived communities in coastal plankton. Nat. Commun. 9, 266 (2018).

37. P. Arevalo, D. VanInsberghe, J. Elsherbini, J. Gore, M. F. Polz, A reverse ecology approach based on a biological definition of microbial populations. Cell. 178, 820-834 (2019).

38. M. Delbrück, The growth of bacteriophage and lysis of the host. J. Gen. Physiol. 23, 643-660 (1940).

39. Supplementary results, materials, and methods are available as supplementary materials on Science Online.

40. O. X. Cordero, M. F. Polz, Explaining microbial genomic diversity in light of evolutionary ecology. Nat. Rev. Microbiol. 12, 263-273 (2014).

41. B. J. Shapiro, J. Friedman, O. X. Cordero, S. P. Preheim, S. C. Timberlake, G. Szabó, M. F. Polz, E. J. Alm, Population genomics of early events in the ecological differentiation of bacteria. Science. 336, 48-51 (2012).

42. E. R. Westra, S. van Houte, S. Oyesiku-Blakemore, B. Makin, J. M. Broniewski, A. Best, J. Bondy-Denomy, A. Davidson, M. Boots, A. Buckling, Parasite exposure drives selective evolution of constitutive versus inducible defense. Curr. Biol. 25, 1043-1049 (2015).

43. S. Zborowsky, D. Lindell, Resistance in marine cyanobacteria differs against specialist and generalist cyanophages. Proc. Natl. Acad. Sci., U.S.A. 116, 16899-16908 (2019).

44. E. O. Alseth, E. Pursey, A. M. Luján, I. McLeod, C. Rollie, E. R. Westra, Bacterial biodiversity drives the evolution of CRISPR-based phage resistance. Nature. 574, 549-552 (2019).

340 45. C. A. Hernandez, B. Koskella, Phage resistance evolution in vitro is not reflective of in vivo outcome in a plantbacteria-phage system. Evolution. 73, 2461-2475 (2019).

46. K. E. Dingle, D. Griffiths, X. Didelot, J. Evans, A. Vaughan, M. Kachrimanidou, N. Stoesser, K. A. Jolley, T. Golubchik, R. M. Harding, T. E. Peto, W. Fawley, A. S. Walker, M. Wilcox, D. W. Crook, Clinical Clostridium difficile: Clonality and pathogenicity locus diversity. PLoS One. 6, 19993 (2011).

47. B. J. Shapiro, How clonal are bacteria over time? Curr. Opin. Microbiol. 31, 116-123 (2016). 
48. Y. Yin, W. Tan, G. Wang, S. Kong, X. Zhou, D. Zhao, Y. Jia, Z. Pan, X. Jiao, Geographical and longitudinal analysis of Listeria monocytogenes genetic diversity reveals its correlation with virulence and unique evolution. Microbiol. Res. 175, 84-92 (2015).

49. J. R. Thompson, S. Pacocha, C. Pharino, V. Klepac-Ceraj, D. E. Hunt, J. Benoit, R. Sarma-Rupavtarm, D. L. Distel, M. F. Polz, Genotypic Diversity Within a Natural Coastal Bacterioplankton Population. Science. 307, 1311-1313 (2005).

50. Code is provided in Data S2 and online (DOI: 10.5281/zenodo.5228182).

51. Supporting data for phylogenetic trees provided in Data S9 and online (DOI: 10.5061/dryad.sj3tx965k).

52. D. E. Hunt, L. A. David, D. Gevers, S. P. Preheim, E. J. Alm, M. F. Polz, Resource partitioning and sympatric differentiation among closely related bacterioplankton. Science. 320, 1081-1085 (2008).

53. S. G. John, C. B. Mendez, L. Deng, B. Poulos, A. K. M. Kauffman, S. Kern, J. Brum, M. F. Polz, E. A. Boyle, M. B. Sullivan, A simple and efficient method for concentration of ocean viruses by chemical flocculation. Environ. Microbiol. Rep. 3, 195-202 (2011).

54. K. M. Kauffman, M. F. Polz, Streamlining standard bacteriophage methods for higher throughput. MethodsX. 5, 159-172 (2018).

55. N.F. Alikhan, N. K. Petty, N. L. Ben Zakour, S. A. Beatson, BLAST ring image generator (BRIG): simple prokaryote genome comparisons. BMC Genomics. 12, 402 (2011).

56. L. A. Kelley, S. Mezulis, C. M. Yates, M. N. Wass, M. J. E. Sternberg, The Phyre2 web portal for protein modeling, prediction and analysis. Nat. Protoc. 10, 845-858 (2015).

57. J. Huerta-Cepas, K. Forslund, L. P. Coelho, D. Szklarczyk, L. J. Jensen, C. von Mering, P. Bork, Fast genomewide functional annotation through orthology assignment by eggNOG-Mapper. Mol. Biol. Evol. 34, 21152122 (2017).

58. J. Huerta-Cepas, D. Szklarczyk, D. Heller, A. Hernández-Plaza, S. K. Forslund, H. Cook, D. R. Mende, I. Letunic, T. Rattei, L. J. Jensen, C. von Mering, P. Bork, eggNOG 5.0: a hierarchical, functionally and phylogenetically annotated orthology resource based on 5090 organisms and 2502 viruses. Nucleic Acids Res. 47, 309-314 (2019).

59. L. Guy, J. R. Kultima, S. G. E. Andersson, genoPlotR: comparative gene and genome visualization in R. Bioinformatics. 26, 2334-2335 (2010).

60. M. Steinegger, J. Söding, MMseqs2 enables sensitive protein sequence searching for the analysis of massive data sets. Nat. Biotechnol. 35, 1026-1028 (2017).

61. J. P. Meier-Kolthoff, M. Göker, VICTOR: genome-based phylogeny and classification of prokaryotic viruses. Bioinforma. Oxf. Engl. 33, 3396-3404 (2017).

62. P. Jones, D. Binns, H.-Y. Chang, M. Fraser, W. Li, C. McAnulla, H. McWilliam, J. Maslen, A. Mitchell, G. Nuka, S. Pesseat, A. F. Quinn, A. Sangrador-Vegas, M. Scheremetjew, S.-Y. Yong, R. Lopez, S. Hunter, InterProScan 5: genome-scale protein function classification. Bioinformatics. 30, 1236-1240 (2014).

63. S. R. Eddy, Accelerated profile HMM searches. PLoS Comput. Biol. 7, 1002195 (2011), doi:10.1371/journal.pcbi.1002195.

64. C. Camacho, G. Coulouris, V. Avagyan, N. Ma, J. Papadopoulos, K. Bealer, T. L. Madden, BLAST+: architecture and applications. BMC Bioinformatics. 10, 421 (2009). 
65. M. Baym, S. Kryazhimskiy, T. D. Lieberman, H. Chung, M. M. Desai, R. Kishony, Inexpensive multiplexed library preparation for megabase-sized genomes. PLoS One. 10, 0128036 (2015).

66. https://github.com/rrwick/Filtlong.

67. M. Kolmogorov, J. Yuan, Y. Lin, P. A. Pevzner, Assembly of long, error-prone reads using repeat graphs. Nat. Biotechnol. 37, 540-546 (2019).

68. R. R. Wick, M. B. Schultz, J. Zobel, K. E. Holt, Bandage: interactive visualization of de novo genome assemblies. Bioinformatics. 31, 3350-3352 (2015).

69. http://www.bioinformatics.babraham.ac.uk/projects/trim_galore/.

70. R. R. Wick, L. M. Judd, C. L. Gorrie, K. E. Holt, Unicycler: Resolving bacterial genome assemblies from short and long sequencing reads. PLoS Comput. Biol. 13, 1005595 (2017).

71. D. Hyatt, G.L. Chen, P. F. LoCascio, M. L. Land, F. W. Larimer, L. J. Hauser, Prodigal: prokaryotic gene recognition and translation initiation site identification. BMC Bioinformatics. 11, 119 (2010).

72. T. J. Treangen, B. D. Ondov, S. Koren, A. M. Phillippy, The Harvest suite for rapid core-genome alignment and visualization of thousands of intraspecific microbial genomes. Genome Biol. 15, 524 (2014).

73. A. K. M. Kauffman, thesis, Massachusetts Institute of Technology (2014). https://dspace.mit.edu/handle/1721.1/90046.

74. K. Katoh, D. M. Standley, MAFFT multiple sequence alignment software version 7: Improvements in performance and usability. Mol. Biol. Evol. 30, 772-780 (2013).

75. A. Stamatakis, RAxML version 8: a tool for phylogenetic analysis and post-analysis of large phylogenies. Bioinformatics. 30, 1312-1313 (2014).

76. S. V. Angiuoli, S. L. Salzberg, Mugsy: fast multiple alignment of closely related whole genomes. Bioinforma. Oxf. Engl. 27, 334-342 (2011).

77. R Core Team, R: A language and environment for statistical computing (2019). https://www.R-project.org.

78. G. Marçais, C. Kingsford, A fast, lock-free approach for efficient parallel counting of occurrences of k-mers. Bioinformatics. 27, 764-770 (2011).

79. B. Langmead, S. L. Salzberg, Fast gapped-read alignment with Bowtie 2. Nat. Methods. 9, 357-359 (2012).

80. B. D. Ondov, T. J. Treangen, P. Melsted, A. B. Mallonee, N. H. Bergman, S. Koren, A. M. Phillippy, Mash: fast genome and metagenome distance estimation using MinHash. Genome Biol. 17, 132 (2016).

81. M. Bastian, S. Heymann, M. Jacomy, paper presented at the Third International AAAI Conference on Weblogs and Social Media, San Jose, CA, 17-20 May 2009.

82. L. Zimmermann, A. Stephens, S.-Z. Nam, D. Rau, J. Kübler, M. Lozajic, F. Gabler, J. Söding, A. N. Lupas, V. Alva, A Completely Reimplemented MPI Bioinformatics Toolkit with a New HHpred Server at its Core. $J$. Mol. Biol. 430, 2237-2243 (2018).

83. S. F. Altschul, T. L. Madden, A. A. Schäffer, J. Zhang, Z. Zhang, W. Miller, D. J. Lipman, Gapped BLAST and PSI-BLAST: a new generation of protein database search programs. Nucleic Acids Res. 25, 3389-3402 (1997). 
84. L. Fu, B. Niu, Z. Zhu, S. Wu, W. Li, CD-HIT: accelerated for clustering the next-generation sequencing data. Bioinformatics. 28, 3150-3152 (2012).

85. I. A. Murray, T. A. Clark, R. D. Morgan, M. Boitano, B. P. Anton, K. Luong, A. Fomenkov, S. W. Turner, J. Korlach, R. J. Roberts, The methylomes of six bacteria. Nucleic Acids Res. 40,11450-11462 (2012).

86. R. J. Roberts, T. Vincze, J. Posfai, D. Macelis, REBASE--a database for DNA restriction and modification: enzymes, genes and genomes. Nucleic Acids Res. 43, D298-299 (2015).

87. L. Ferrières, G. Hémery, T. Nham, A.-M. Guérout, D. Mazel, C. Beloin, J.-M. Ghigo, Silent mischief: Bacteriophage mu insertions contaminate products of Escherichia coli random mutagenesis performed using suicidal transposon delivery plasmids mobilized by broad-host-range RP4 conjugative machinery. $J$. Bacteriol. 192, 6418-6427 (2010).

88. S. L. Chiang, E. J. Rubin, Construction of a mariner-based transposon for epitope-tagging and genomic targeting. Gene. 296, 179-185 (2002).

89. F. Le Roux, J. Binesse, D. Saulnier, D. Mazel, Construction of a Vibrio splendidus mutant lacking the metalloprotease gene vsm by use of a novel counter-selectable suicide vector. Appl. Environ. Microbiol. 73, 777-784 (2007).

90. S. Das, J. C. Noe, S. Paik, T. Kitten, An improved arbitrary primed PCR method for rapid characterization of transposon insertion sites. J. Microbiol. Methods. 63, 89-94 (2005).

91. F. M. Lauro, K. Tran, A. Vezzi, N. Vitulo, G. Valle, D. H. Bartlett, Large-scale transposon mutagenesis of Photobacterium profundum SS9 reveals new genetic loci important for growth at low temperature and high pressure. J. Bacteriol. 190, 1699-1709 (2008).

92. Y. Jiao, A. Kappler, L. R. Croal, D. K. Newman, Isolation and characterization of a genetically tractable photoautotrophic Fe(II)-oxidizing bacterium, Rhodopseudomonas palustris Strain TIE-1. Appl. Environ. Microbiol. 71, 4487-4496 (2005).

93. M.-E. Val, O. Skovgaard, M. Ducos-Galand, M. J. Bland, D. Mazel, Genome engineering in Vibrio cholerae: A feasible approach to address biological issues. PLoS Genet. 8, 1002472 (2012).

94. A. Lemire, D. Goudenège, T. Versigny, B. Petton, A. Calteau, Y. Labreuche, F. Le Roux, Populations, not clones, are the unit of vibrio pathogenesis in naturally infected oysters. ISME J. 9, 1523-1531 (2015).

95. R. C. Edgar, MUSCLE: multiple sequence alignment with high accuracy and high throughput. Nucleic Acids Res. 32, 1792-1797 (2004).

450 96. H. Wildschutte, S. P. Preheim, Y. Hernandez, M. F. Polz, O-antigen diversity and lateral transfer of the wbe region among Vibrio splendidus isolates. Environ. Microbiol. 12, 2977-2987 (2010).

97. J. Söding, A. Biegert, A. N. Lupas, The HHpred interactive server for protein homology detection and structure prediction. Nucleic Acids Res. 33, W244-W248 (2005).

Acknowledgments: We thank M. Cutler for experimental support. We thank D. Newman, K.

Costa, and H. Wildschutte for advice on mutagenesis experiments. We thank O. X. Cordero, S. 
Kearney, and A. F. Takemura for valuable suggestions throughout. We thank S. W. Chisholm, L. Kelly, and S. Pollak for thoughtful comments on the manuscript. Finally, we thank D. Kwon, X. $\mathrm{Yu}, \mathrm{O}$. Abudayyeh, and J. Gootenberg for use of ddPCR equipment and/or lab space during the SARS-CoV-2 pandemic.

Funding: Simons Foundation (Life Sciences Project Award-572792) (MFP); National Science Foundation Division of Ocean Sciences (OCE-1435868) (MFP); MIT J-WAFS seed grant (MFP); NSF GRFP (FAH); MIT Martin Society of Fellows for Sustainability (FAH); Xunta de Galicia Postdoctoral Fellowship (ED481B 2016/032) (JD); Agence Nationale de la Recherche 465 (ANR-16-CE32-0008-01) (FLR); European Research Council (ERC) under the European Union's Horizon 2020 research and innovation programme (grant agreement No 884988, Advanced ERC Dynamic) (FLR)

Author contributions: Conceptualization: FAH, MFP. Data Curation: FAH, with contributions from JE, JD, MM, PA, DV, BK, KK. Methodology: FAH, JD, MM, JE, FLR. Investigation: 470 FAH, JD, FLR, with contributions from JE, MM, PA, DV, KK, BK, HG. Validation: FAH, JD, FLR, with contributions from JE, HG, AL, AG. Visualization: FAH, JE with contributions from DV, PA, KK. Software: FAH, JE, MM, with contributions from DV, PA. Funding acquisition: MFP, FLR, FAH, JD. Project administration and supervision: MFP, FLR. Writing - original draft: FAH, MFP. Writing - review \& editing: FAH, MFP with contributions from JD, FLR.

475 Competing interests: Authors declare that they have no competing interests.

Data and materials availability: New genomes used in this work have been deposited under the NCBI BioProject with accession number PRJNA328102. Main code is provided in Data S2 and online (50). Supporting data provided in Data S9 and online (51). 
$480 \quad$ List of Supplementary materials:

Materials and Methods

Figures S1 to S22

Tables S1 to S4

References (52-97)

$485 \quad$ Data S1 to S9

490 


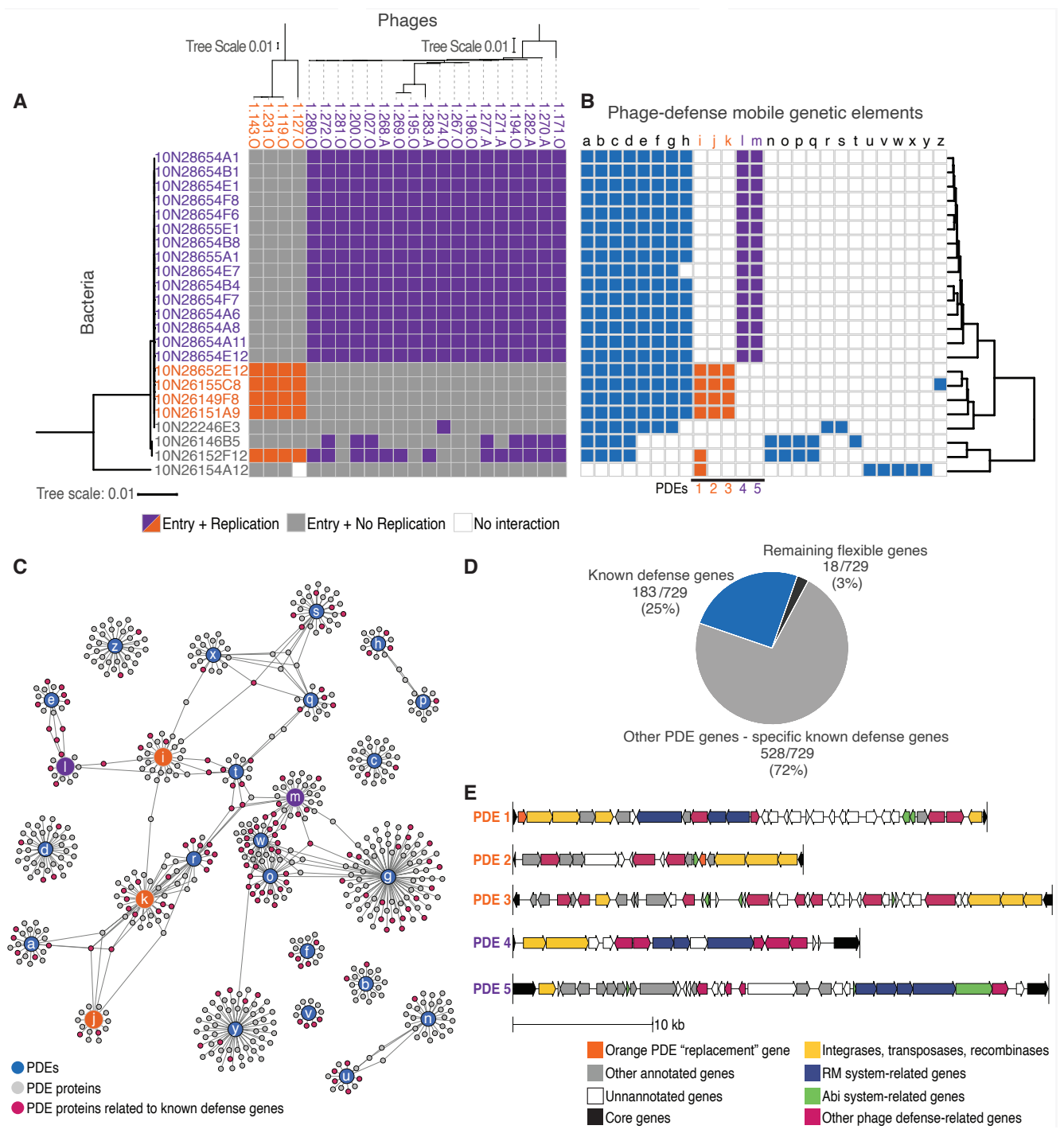

Fig. 1. Near-clonal strains of Vibrio lentus differ in sensitivity to phage predation and in their carriage of mobile genetic elements encoding for phage defense genes. (A) Phage hostrange matrix with rows representing bacterial strains and columns representing phages. (A-left) Core genome phylogenetic tree of hosts, (A-top) whole genome tree of viruses. (B) Distribution of 26 PDEs in 23 hosts with PDEs responsible for defense of the "orange" and "purple" host groups indicated in the same colors, (B-right) hierarchical clustering of presence/absence of genomic regions in bacterial hosts corroborating "orange" and "purple" phage infection patterns. (C) Shared genes ( $>50 \%$ amino acid sequence identity) among the 26 PDEs displayed as a network where gray nodes are genes with defense genes indicated in pink. (D) Fraction of the bacterial flexible genome attributed to phage defense. Amongst the 23 clones, an all-by-all genomic comparison shows $97 \%$ of flexible regions greater than $5 \mathrm{kbp}$ are putative PDEs. Only $25 \%$ of the total flexible genes match known defense genes while the majority are other PDEassociated genes $(72 \%)$, many of which are unannotated. The remaining genes, not associated with PDEs, account for 3\% of the flexible genome. (E) Gene diagrams of mobile genetic elements specific to the "orange" and "purple" host groups. 
A

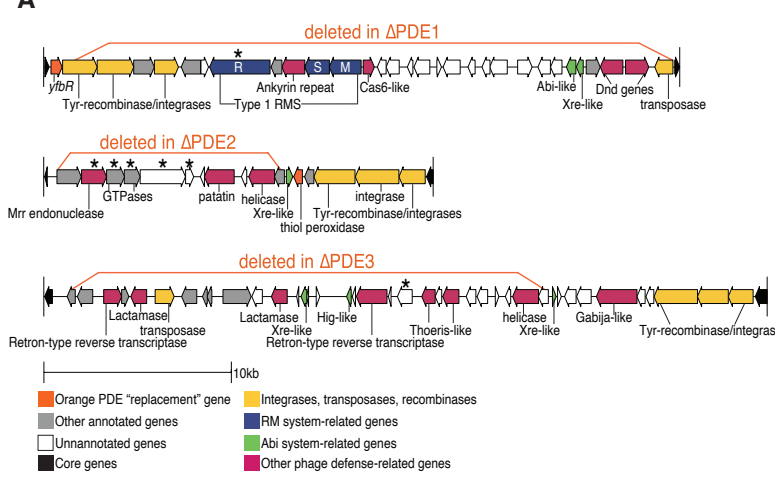

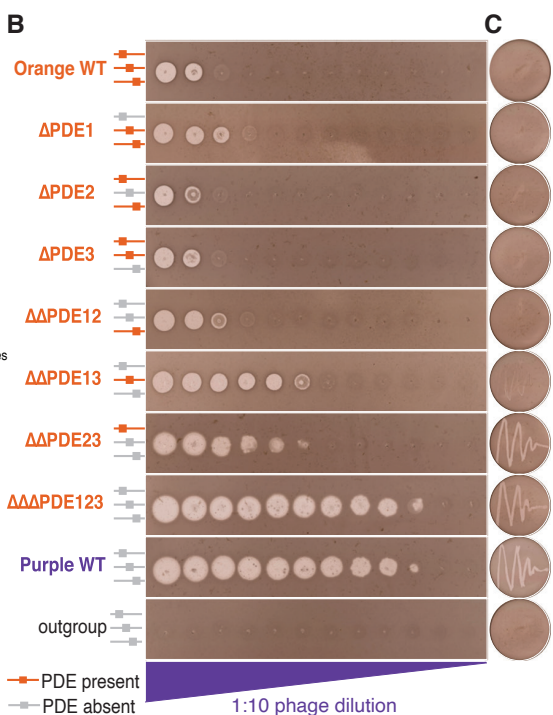

Fig. 2. Changes in susceptibility to phage killing observed for phage defense element (PDE) deletions. (A) Detailed gene diagrams indicate marker-less deletions of defense-gene containing portions of the different "orange" PDEs as well as genes determined to be responsible for defense phenotypes (marked with *, Figs. S15-17). (B) Lawns of bacterial hosts with drop spots of a 1:10 dilution series of "purple" phage (image shows representative "purple" phage 1.281.O; plating completed in triplicate for each phage, Fig. S12). Cartoons on the left depict presence or absence of different PDEs in each strain. From top to bottom: "orange" wild type host (10N26155C8), $\triangle \mathrm{PDE} 1, \Delta \mathrm{PDE} 2, \Delta \mathrm{PDE} 3, \Delta \Delta \mathrm{PDE} 12, \Delta \Delta \mathrm{PDE} 13, \Delta \Delta \mathrm{PDE} 23, \Delta \Delta \Delta \mathrm{PDE} 123$, "purple" wild type host (10N28654F7, positive control), outgroup (10N26149C11, negative control). (C) Re-streak test for propagation of phage progeny from drop spot clearings. Only infections of $\Delta \Delta \mathrm{PDE} 23, \Delta \Delta \Delta \mathrm{PDE} 123$, and "purple" wild type hosts produce viable phages, indicated by clearing on the re-streak plates. 


\section{Supplementary Materials for}

\section{Rapid evolutionary turnover of mobile genetic elements drives bacterial resistance to phages}

Fatima Aysha Hussain, Javier Dubert, Joseph Elsherbini, Mikayla Murphy, David VanInsberghe, Philip Arevalo, Kathryn Kauffman, Bruno Kotska Rodino-Janeiro, Hannah Gavin, Annika

Gomez, Anna Lopatina, Frédérique Le Roux*, and Martin F. Polz*

*Correspondence to: martin.f.polz@univie.ac.at and fleroux@sb-roscoff.fr

\section{Materials and Methods}

Bacteria and phage isolation

Bacteria and phages were obtained in a previous study from coastal seawater collected from Canoe Cove, Nahant, MA, USA, on August 22 (ordinal day 222), September 18 (261), and October 13 (286), 2010 (34). Vibrio bacteria were isolated using a size fractionation approach, followed by plating on selective media, as described previously (52). Briefly, to capture bacteria associated with large particles and zoo- and phytoplankton, seawater was filtered through a 63 $\mu \mathrm{m}$ average pore size plankton net. To capture bacteria in smaller size fractions, including small particles and smaller zoo- and phytoplankton as well as bacteria occurring in the free-living fraction, water pre-filtered through a $63 \mu \mathrm{m}$ net was serially passed through $5 \mu \mathrm{m}, 1 \mu \mathrm{m}$, and 0.2 $\mu \mathrm{m}$ polycarbonate filters. To isolate vibrios from each fraction, material captured in the plankton net and on filters was resuspended in artificial seawater (ASW; Sea Salts from Sigma-Aldrich), and the suspensions passed through polyether sulfone $0.2 \mu \mathrm{m}$ filters. These final filters were placed directly on agar plates of MTCBS (Difco Thiosulfate-Citrate-Bile-Sucrose Agar amended with $10 \mathrm{~g} / \mathrm{L}$ of $\mathrm{NaCl}$ to final concentration of $2 \% \mathrm{w} / \mathrm{v}$ ) to allow for selective growth of Vibrio colonies. Colonies were purified by serial passaging on agar plates of: first, TSB2 (Tryptic Soy Broth, 1.5\% Difco Bacto Agar, amended with $15 \mathrm{~g} \mathrm{NaCl}$ to $2 \% \mathrm{w} / \mathrm{v}$ ); second, MTCBS, and third TSB2. Colonies were inoculated into $1 \mathrm{~mL}$ of Difco 2216 Marine Broth (2216MB) in 96-well 2 $\mathrm{mL}$ culture blocks and allowed to grow, shaking at room temperature, for 48 hours. Glycerol stocks for preservation at $-80^{\circ} \mathrm{C}$ were prepared by combining $100 \mu \mathrm{L}$ of culture with $100 \mu \mathrm{L}$ of $50 \%$ glycerol (50\% water) in 96-well microtiter plates. The naming of each strain reflects isolation location, day, and size fraction: $10 \mathrm{~N}$ refers to the 2010 collection of samples from Nahant; 222, 261, 286 are the ordinal dates of the year; 54, 55, 56 are three replicates of the 63 $\mu \mathrm{m}$ fraction; 51, 52, 53, are three replicates of the $5 \mu \mathrm{m}$ fraction; 48, 49, 50 are three replicates of the $1 \mu \mathrm{m}$ fraction; and 45, 46, 47 are three replicates of the $0.2 \mu \mathrm{m}$ or free-living fraction. The final portion of the name is the original storage well in a 96-well plate. The "orange" phage isolates were, with one exception, collected on day 261 and distributed between the $1 \mu \mathrm{m}, 5 \mu \mathrm{m}$, and $63 \mu \mathrm{m}$ fraction, while all "purple" isolates were collected on day 286 from the $63 \mu \mathrm{m}$ fraction. This isolation scheme reflects a snapshot of the lifestyle of the isolated bacteria, where $5 \mu \mathrm{m}$ and $63 \mu \mathrm{m}$ represent living in a particle-attached state in the ocean.

For phage isolation, $4 \mathrm{~L}$ of seawater was collected in triplicate on each day in the time series and separately filtered through a Sterivex $0.22 \mu \mathrm{m}$ barrel filter into a sterile $4 \mathrm{~L}$ collection bottle using a peristaltic pump. Phages were directly concentrated from this filtrate using an iron flocculation and filtering method described previously (53). Briefly, iron (III) chloride, which is spiked into the sample, precipitates phages from the solution, and then the precipitates are 
collected onto $90 \mathrm{~mm} 0.2 \mu \mathrm{m}$ polycarbonate filters using a glass cup-frit system. Precipitates are finally dissolved in $4 \mathrm{~mL}$ of oxalate solution to yield a quantitative concentration of 1,000x from the original $4 \mathrm{~L}$. The final phage concentrate was stored at $4^{\circ} \mathrm{C}$ in the dark until used to isolate specific viruses for different bacterial hosts.

Vibrio isolates were used as "bait" to obtain phages from the concentrates using direct plating in soft agar overlays. Plaques from the bait assay were archived frozen in $2216 \mathrm{MB}$ and glycerol and phages for use in the host range assay were subsequently randomly selected from archives for each host and purified by triple serial passage using tube-free agar overlays $(33,54)$ on their hosts of isolation. Phages were amplified on their hosts of isolation using primary smallscale liquid cultures inoculated with plaque plugs from their final serial passage in agar overlays, followed by plating of primary lysates into agar overlays to achieve high-titer stocks. Top agar layers of lawns "at confluence" (saturated with plaques but not completely cleared) were harvested into $2216 \mathrm{MB}$, centrifuged at 5,000 x $g$ for 20 minutes, and filtered through Sterivex $0.22 \mu \mathrm{m}$ barrel filters to generate the lysates used for the all-by-all host range cross test as well as for phage DNA extraction and sequencing (33), as well as methylation profiling (described below).

\section{Phage characterization}

Circular representations of the previously sequenced phage genomes (Fig. S2CD) were generated using BRIG (55) with publicly available NCBI GenBank files; annotations were made based on manual review of GenBank predictions and supplemented with Phyre2 (56) and EggNog-Mapper $(57,58)$ annotation. Genome diagrams (Fig. S2EF) were generated using the GenoPlotR package in $\mathrm{R}(59)$ with predicted protein coding genes indicated as arrows colored to correspond to protein sequence clusters, as defined using default settings of MMseqs2 (60); and with "orange" (Fig. S2E) and "purple" (Fig. S2F) phages clustered and identified as two separate genus-level groups using the D6 amino acid OPTSIL clustering algorithm in the VICTOR classifier (61) with whole genome concatenated protein sequences.

Although siphoviruses are frequently temperate, there is no evidence the "orange" and "purple" phages integrate into bacterial genomes, nor of genetic features associated with a temperate life history strategy (Data S1). All phages in the Nahant Collection were evaluated for lysogeny associated gene features and presence as prophages in bacterial genome sequences previously (35) and verified here. No such evidence was found for any of the "purple" or "orange" phages. Evaluation for lysogeny associated genes: EggNOG-mapper v.2 (58) and InterProScan v.5.39-77.0 (62) annotations for all phages were reviewed for integrases (PF00589, PF00239), replicative transposases (PF02914), and transcriptional repressor domains (IPR010982, IPR010744, IPR001387, IPR032499); iterative sequence-based searches using the jackhmmer function of HMMER v.3.1.2 (63) were used to screen for plasmid associated genes SopA (NP_046923.1) and ParA (AAA99230.1). No such genes were found for any "orange" or "purple" phages. Evaluation for integration as prophages: BLASTn (64) searches were used to screen 309 Nahant Collection bacterial genome assemblies for sequences with significant similarity to those of the "orange" and "purple" phages. No significant hits (e-value $\leq 1.00 \mathrm{E}-05$ ) were found between any of the phages and the bacterial genomes (Data S1).

Hybrid assemblies of bacterial genomes

Because we noticed that PDEs assemble poorly when genomes were sequenced with short read technology, we used Illumina short reads and Pacific Biosciences (PacBio) long reads in 
combination to assemble high quality (nearly closed) genomes. For short read sequencing, bacterial isolates were grown overnight from a single colony in $1.2 \mathrm{~mL}$ of $2216 \mathrm{MB}$ in deep-well blocks and processed in bulk. Genome libraries were prepared for sequencing using the Nextera DNA Library Preparation Kit (Illumina) with 1-2 ng input DNA per isolate, as previously described (65). Genomes were sequenced on $100 \mathrm{bp}$ pared-end sequencing runs using Illumina HiSeq, with 50-60 samples multiplexed per lane. When available, Illumina HiSeq short reads from previous work (34) were used, otherwise new short read data were generated for this study. High quality bacterial genomic DNA for PacBio sequencing was prepared separately. A single colony was inoculated into a $250 \mathrm{~mL}$ Erlenmeyer flask with $50 \mathrm{~mL}$ of $2216 \mathrm{MB}$ and grown shaking at room temperature for 24 hours. The fresh culture was pelleted by centrifugation at $5,000 \mathrm{x} g$ for 20 minutes and then immediately processed for DNA extraction when possible, or frozen at $-20^{\circ} \mathrm{C}$ for short term storage. The Qiagen Genomic Tip 500x kit was used following the manufactures guidelines, and the final DNA was collected by spooling using a glass rod rather than centrifugation to avoid shearing. DNA was stored in $500 \mu \mathrm{L}$ of elution buffer at $4^{\circ} \mathrm{C}$ for 24 48 hours to allow for full resuspension before sequencing at either the Yale Center for Genome Analysis (PacBio RS II, without multiplexing) or the BioMicroCenter at MIT (Sequel, with multiplexing).

A custom hybrid assembly pipeline was designed to process the data. Briefly, PacBio reads were filtered at different length cutoffs using Filtlong (66) and then assembled using Flye (67) to create a set of reference genomes. The reference genomes were visualized using Bandage (68) and the best genome was selected, based on completion and coverage, to be used as a reference in the final assembly. For the final assembly, Illumina reads were trimmed using TrimGalore (69), PacBio reads were quality filtered with the trimmed Illumina reads using Filtlong, and both sets of processed reads, together with the best Flye assembly, were used as inputs for

115 the Unicycler (70) assembler.

\section{Bacterial genome annotations}

Genomes were annotated using Prodigal 2.6 (71) for Open Reading Frame (ORF) prediction. Predicted ORFs were annotated using InterProScan5 (62) using the iprlookup, goterms, and pathways options. InterProScan5 matches against 13 databases by default, which are listed here: https://github.com/ebi-pf-team/interproscan/wiki/HowToRun\#included-analyses. Two optional databases were included for this analysis: TMHMM for predicted transmembrane proteins and SignalP for predicted signal peptide cleavage sites.

$125 \quad$ Host relationships

Phylogenetic relationships among the Vibrio strains were estimated by comparison of (i) concatenated alignments of ribosomal proteins and $h s p 60$ as described below in methods for, "Phage host-range matrix" (Fig. S1A), and (ii) core genome alignments. For estimation of whole genome relationships, we used the Parsnp program (72) with the recombination flag (-x) to

130 construct whole genome SNP trees. Then, HarvestTools was used to convert from a ggr format to a snp fasta file, and finally, IQ-Tree was used to optimize the final tree. This was done for the 23 clones (Fig. 1A, left) as well as for the "purple" and "orange" strains alone (Fig. S8). SNPs in the core genome were located using custom python scripts and verified by visualizing on Ginger, the Harvest graphic user interface. SNP details for Fig. S8 are given in Table S3. 
Initial phage host-range was determined in a previous study $(34,35)$. Briefly, all Vibrio strains for which at least one phage was found ("plaque positive") were used in the host-range assay and challenged with all phages purified as described above. Bacterial hosts were plated in agar overlays in large $150 \mathrm{~mm}$ plates and stamped with phage lysates arranged in triplicate in 96-well arrays using 96-spot blotters (BelArt, Bel-blotter 96-tip replicator, 378760002). Clearing seen in at least $2 / 3$ replicates was scored as a positive kill (73). The concentration of each lysate in the original assay was not normalized to allow for higher throughput, however, the assay was repeated for select hosts of interest at a range of concentrations (see methods for "varying phage concentrations" below).

To organize bacterial hosts in the matrix by phylogeny, concatenation of ribosomal proteins and $h s p 60$ sequences was used to construct a phylogenetic tree reflecting the relationship of the core genome (Fig. S1A). When genome sequences were available, we used HMMER (63) to find ribosomal proteins, and aligned the sequences with MAFFT (74). Amino acid sequences of $h s p 60$ proteins were also extracted from genomes via HMMER using pfam PF00118. The $h s p 60$ sequences were aligned using the mafft-fftnsi algorithm. When genomes were not available, $h s p 60$ sequences that were Sanger-sequenced were added to this alignment using the mafft-fftnsi algorithm with the -addfragments option. The $h s p 60$ alignment and the ribosomal protein alignment were concatenated and used to create the phylogenetic tree in Figure S1A using RAxML (options: -q, -m GTRGAMMAX) (Data S9) (75).

Host range assays at varying phage concentrations

To determine the host-ranges of the specific phages used in this work at a higher resolution, we re-assayed a subset of hosts and phages of interest using a range of concentrations. Bacterial hosts were grown in $5 \mathrm{~mL}$ of $2216 \mathrm{MB}$ overnight from single colonies streaked on $1.5 \%$ Bacto Agar plates supplemented with 2216MB. Phage lysates were prepared as described above and diluted in $2216 \mathrm{MB}$ to form a ten-fold dilution series from $10^{0}-10^{-7}$. Five $\mu \mathrm{L}$ drop spots of each dilution were pipetted onto bacterial host lawns made using a tube-free agar overlay method (54) and incubated at room temperature for 24 hours before evaluating phage entry and efficiency of plating at the varying concentrations (Fig.1A). Plates with different killing were imaged using a flatbed scanner (Epson Perfection V800 Photo Scanner - Product No. B11B22320) and captured using VueScan Software by Hamrick (Fig. S3). Lysis from without was only observed when using this higher resolution assay, thus the authors recommend performing such an assay when evaluating phage host-range whenever possible.

Phage adsorption assay

To determine if all phages were attaching to all hosts, for "orange" phage 1.143.O and "purple" phage 1.281.O, we compared the number of free phages remaining in solution after exposure to "orange" host 10N26155C8, "purple" host 10N28654F7, an unrelated Vibrio (outgroup) control $10 \mathrm{~N} 26149 \mathrm{C} 11$, and a no-host negative control (2216MB). Three different colonies of each bacterial strain were each inoculated in $3 \mathrm{~mL}$ of $2216 \mathrm{MB}$ and grown shaking at $25^{\circ} \mathrm{C}$ for 4 hours. Bacterial concentration was estimated at optical density measured at $600 \mathrm{~nm}$ wavelength $\left(\mathrm{OD}_{600}\right)$, and each replicate was normalized to $\mathrm{OD}_{600}$ of 0.3 followed by 100 -fold dilution. One $\mathrm{mL}$ of each diluted culture was aliquoted into individual wells of a 96-well culture block and bacteria were grown shaking at room temperature for another 3.5 hours to reach mid-exponential phase. Twenty $\mu \mathrm{L}$ of phage lysate was added to each well at varying concentrations (ranging from $\mathrm{MOI}=0.001$ to $10 \mathrm{phages} /$ bacteria on average) and staggered in time to achieve an 
adsorption time of 30 minutes (Fig. S4). After allowing phages to adsorb, $200 \mu \mathrm{L}$ of the phage and bacteria mixture was filtered using a 96-well filter system (Millipore MultiScreen Vacuum 185 Manifold) to remove bacteria and any infecting or adsorbed phages. Five $\mu \mathrm{L}$ of a ten-fold dilution series of each well was then drop spotted onto a fresh lawn of a sensitive host (orange strain 10N26155C 8 was used for experiments with 1.143.O and 10N28654F7 was used for experiments with 1.281.O) made in rectangular petri dishes (1-well Nunc Rectangular Dishes, Polystyrene, Sterile by Thermo Scientific (Supplier No. 267060)). Plates were incubated at room 190 temperature for 18-24 hours and then imaged using a flatbed scanner as described above (Fig. S4). Phage adsorption was estimated by comparing the number of plaque forming units (PFUs) in each dilution series to the no host control. For example, in Figure S4A the same order of magnitude of PFUs is evident in both the outgroup and the no-host control, meaning there is no phage adsorption for the outgroup. Yet, there are an order of magnitude more PFUs in both 195 controls compared to the "purple" and "orange" hosts, implying equal adsorption is seen on the "purple" and "orange" hosts.

Identification, annotation, and characterization of putative PDEs in the flexible genome In order to determine the differences in the flexible genome amongst the 23 clonal strains (Fig. 200 1), we created a multiple alignment using Mugsy (76), and performed a hierarchical clustering of the alignment blocks, greater than 500 bp, by length in R using hclust (77) (Fig.1B, right).

Then to specifically identify the exact regions of interest in the flexible genome, we used a k-mer based approach (Data S2), followed by careful manual curation. All pairs of genomes were compared. First, each genome was split into 31-mers using Jellyfish (78), then shared 205 kmers between the genomes being compared were removed and only unique kmers were mapped back to the reference genomes they originated from using Bowtie2 (79). Any unique region greater than 1,000 bp was kept and a gap of 3,000 bp was allotted to account for genes that may have been shared between the two genomes splitting a complete region. Regions were checked for duplication and the largest region of any overlapping regions was saved. We then used Mash 210 (80) to compare all the unique regions to each other and clustered any region greater than $5 \mathrm{kbp}$ with minimum Jaccard similarity of 0.95 . We visualized the clustering using Gephi $(81)$ and then chose one representative from each cluster by hand to make a final list of unique regions. We then used BLASTn (64) and custom python scripts to determine which genomes harbored which elements with $>95 \%$ identity and $>80 \%$ length considered a match. We removed any element 215 that appeared in all genomes. Then we went back through the elements and identified insertions sites (see below) to be sure to only count regions moving as a unit. Finally, we used HMMER (63) to search each element for known defense genes using Supplementary Tables 1 and 5 in Doron et al. (15) and Supplementary Tables 1 and 8 in Gao et al. (17). FASTA files for all regions are provided in Data $\mathrm{S} 8$.

220 Gene annotations of the PDEs were performed manually using the consensus obtained from HHPred (82), InterProScan5 (62), Phyre2 (56), and BLASTp (83) databases tools (Data S3). The search was performed using default options except that HHPred search was performed against COG-KOG 1.0 and Pfam-A_v32.0 databases and that BLASTp was performed using the protein-protein BLAST option. Up to ten significant pfam and COG $(\mathrm{p}<0.05)$ from HHPred search where used to compare each gene with pfam-COG accession numbers of phage defense systems from Supplementary Tables 1 and 5 in Doron et al. (15) and Supplementary Tables 1 and 8 in Gao et al. (17). 
Finally, to determine the relationship among the PDEs (Fig. 1C), we clustered the proteins on the PDEs by $50 \%$ identity using CD-HIT (84) and visualized the network using 230 Gephi (81). This low identity threshold only allowed for a limited number of connections, indicating the diversity and unrelatedness of the elements.

Figure 1 depicts a closely related set of 20 strains, which range in divergence from 0-95 SNPs, while on average 757 SNPs differentiate any one of the 20 from the 2 slightly divergent strains and 16,953 SNPs differentiate any one of the 22 from the outgroup. This 235 collection of hosts was essential for the comparative genomics determining PDE turnover. The approach we present here: using comparative genomics and genetics of near clonal isolates combined with phage host-range data is a fruitful method to discover novel phage defense mechanisms in an unbiased way, in addition to monitoring all modes of evolution in response to phage predation (SNP resolution as well as HGT). However, we emphasize that near clonal 240 genomes of high quality are essential since in our experience PDEs assemble poorly when using short read data, in part due to AT richness and high density of variable repeat regions.

\section{Transfer of phage defense elements}

To further characterize the distribution of the 5 specific PDEs identified among the 19 "orange"

245 and "purple" strains in a larger collection of Vibrio genomes (37), we used BLASTn (64) and custom python scripts to identify the distribution of the mobile elements (Fig. S9, Fig. S10). Because when comparing long mobile elements BLASTn will often return multiple overlapping ranges of identity, our BLAST parsing script merges overlapping sequence ranges to avoid overcounting regions within a genome. A PDE was considered present in a genome if at least $80 \%$ of 250 the element was present at over $95 \%$ identity.

Identification of insertion sites

To determine the genomic context for each PDE and find the same region in genomes without the PDE, genes identified using prodigal 2.6 (71) were clustered at $80 \%$ identity using mmseqs 2 (60). For each representative region, we searched for the closest single copy core gene upstream and downstream of the representative PDE. Then for each strain where these core genes were present on the same contig we plotted the intervening genes (Fig. S6).

Proportion of known defense genes in flexible genome across diverse Vibrio

260 To determine the proportion of known defense genes in other Vibrio species, we used the species and population designations from Arevalo et al., (37). We based our identification of flexible genes on the method described in Arevalo et al. ORFs were identified with Prodigal 2.6 (71) and orthologous genes were clustered using MMseqs2 (60). Flexible genes for a given population were defined as orthologs which were present in at least one member but not present in all members of the population. Flexible genes from each genome were then used as a database which we searched for known defense genes using Supplementary Tables 1 and 5 in Doron et al. (15) and Supplementary Tables 1 and 8 in Gao et al. (17) using HMMER (63). Total length of all flexible genes summed for each species and the proportion of genes with a hit to a known defense gene is shown in Figure S7.

Identification of putative PDEs from comparison of closely related genomes of different bacteria We extended the search for novel putative PDEs by searching identify nearly clonal genomes of Salmonella, Listeria, and Clostridia. For Salmonella, and Listeria we selected genomes within 
the same ribotype using the ribosomal MLST database (62), filtered to only include NCBI assemblies, and downloaded genomes from ribotypes with more than 20 members. We used ribotype 8354 for Salmonella and MLST strain type 5 for Listeria to assay putative PDE distribution in an exemplary manner. For Clostridia, we focused on a set of clones with identical ribosomal proteins we hand selected due to limitations in available genome quality. In all cases, the final set of genomes was run though the custom kmer-based comparative genomic pipeline to 280 identify flexible regions (Data S2). All pairs of genomes were compared (see "Identification and annotation of putative PDEs in the flexible genome" for details). As with the Vibrio analysis, we removed any element that appeared in all genomes and used HMMER (63) to search each element for known defense genes using Supplementary Tables 1 and 5 in Doron et al. (15) and Supplementary Tables 1 and 8 in Gao et al. (17). Any unique region with one or more hits was 285 considered to be a putative PDE and depicted in Figure S20, Figure S21, and Figure S22 for Listeria, Salmonella and Clostridium, respectively. Additional annotations were performed with EggNog-Mapper $(57,58)$. FASTA files for all regions are provided in Data S8.

We faced the same issue of genome completion explained above when looking at other vibrio populations as well as the Listeria, Salmonella, and Clostridia analyses, but tried to

290 minimize these issues by looking at closely related (identical ribosomal protein sequences) strains and using the highest quality genomes available.

\section{Proportion of the flexible genome attributed to PDEs}

To determine the proportion of the flexible genome attributed to PDEs in Vibrio, Salmonella,

295 Listeria, and Clostridia, we used the PDE analysis presented for each genus as an exemplar and determined how many genes were attributed to the identified PDEs compared to the number of genes on other flexible regions identified in the k-mer pipeline. The breakdown is included as a separate tab for each group in Data S3-S6.

$300 \quad$ Methylome characterization and restriction-modification activity

To discover if the restriction modification systems identified on the PDEs in the "orange" and "purple" strains are active, we determined the methylation sites in both phage-host pairs as outlined in Murray et al. (85). Briefly, we submitted the host genomes to REBASE, a wellcurated database of restriction modification systems which allows motif prediction based on 305 comparisons to known enzyme-motif pairs (86). Then, we combined the motif data with the methylome data generated using the Base Modification Detection and Motif Analysis pipelines available on the single molecule real-time (SMRT) sequencing portal from Pacific Biosciences. Summary data is presented in Table S4.

Methylome sequencing analysis shows that the distinct sequence motifs predicted for 310 each RM system using REBASE are methylated only within "orange" and "purple" phage-host sets, i.e., only in the bacterial genomes in which the RMs occur, and in the phages that can kill those hosts (Table S4).

To further characterize the escape phenotype of the phages resulting in their ability to kill, we propagated a "purple" phage grown on the triple knockout "orange" host and measured the efficiency of plating on the WT "purple" host (Fig. S14). We saw a reduction of efficiency of plating by seven-fold, yet propagation of plaques was still observed. This is the expected phenotype for a methylated escape phage mutant. Only phages that are inadvertently methylated will be able to kill the WT host, while this methylation is not needed for the triple knock out as it is lacking the corresponding RM system on PDE1. 
Bacterial strain selection for transposon mutagenesis and gene deletions

Because the adsorption assays indicated that both "orange" and "purple" phages adsorbed to both host groups, we chose one strain, 10N26155C8 (Orange WT, hereafter C8-WT), for mapping of receptors for both host groups. For receptor mapping, we took advantage of the lysis from without phenotype where phages can affect lysis if hosts possess a specific receptor even if no viable phage are produced. Accordingly, at high phage titer, cells of both host groups are lysed by both phages (Fig. S3), allowing for testing of receptors using a "purple" phage on an "orange" host. The same C8-WT strain was used for characterization of resistance determinants of the "orange" host group by gene deletion (see below).

Growth conditions of strains used for transposon mutagenesis and gene deletions $\mathrm{C} 8-\mathrm{WT}$ was routinely grown at $25^{\circ} \mathrm{C}$ in $2216 \mathrm{MB}$ or TSB2. The Escherichia coli strains were grown in BD Difco Miller Luria-Bertani broth (LB) at $37^{\circ} \mathrm{C}$ and supplemented for auxotroph strain $E$. coli $\Pi 3813$ with thymidine $(0.3 \mathrm{mM})$, and for strains $E$. coli $\beta 3914$ and MFDpir with diaminopimelic acid (dapA) $(0.3 \mathrm{mM})$. Antibiotics were used at the following concentrations: erythromycin (Erm) $200 \mu \mathrm{g} \mathrm{mL}^{-1}$, kanamycin $(\mathrm{Km}) 50 \mu \mathrm{g} \mathrm{mL}-1$ and chloramphenicol $(\mathrm{Cm})$ at 5 or $25 \mu \mathrm{g} \mathrm{mL}^{-1}$ for Vibrio and E. coli, respectively.

\section{Receptor identification}

340 Transposon mutagenesis as well as resequencing of spontaneously resistant strains all suggest that the "orange" phage receptor is the Type II secretion system pseudopilus (GspH), while the "purple" phages have two receptors, one being the lipopolysaccharide (LPS), an extracellular receptor, and the second being a sodium transporter (NqrC), a membrane receptor (Fig. S5A, Tables S1 and S2). These results are further verified by clean deletions and/or complementation

\section{$345 \quad$ (Fig. S5BC).}

\section{Receptor identification using transposon mutagenesis}

To map phage receptors, transposon mutagenesis was carried out using suicide delivery of a mariner transposon. C8-WT served as the recipient and the dapA deficient strain E. coli MFDpir 350 with the suicide conjugative plasmid pSC189-Cm (87) served as the donor (Data S7). The delivery plasmid (pSC189) can be mobilized via RP4-mediated transfer and it carries the hyperactive $\mathrm{C} 9$ mariner transposase (88). Conjugation was carried out by mating assays as described previously (89) with some modifications. First, donor:recipient ratio was adjusted to 1:3. Overnight cultures were diluted 1:100 in fresh media and grown up to an $\mathrm{OD}_{600}$ of $\sim 0.4$. One

$355 \mathrm{~mL}$ was separately pelleted at 5,500 $\mathrm{g}$ for two minutes and washed in pre-warmed mating media broth MMB-1 (TSB supplemented with $1 \% \mathrm{NaCl}$ plus dapA) to remove antibiotics and/or residual media. This wash step was repeated twice. Washed pellets were subsequently mixed in the same tube with $500 \mu \mathrm{L}$ of MMB-1, pelleted and resuspended in a mating spot $(20 \mu \mathrm{L})$ on mating media agar plates and incubated at $25^{\circ} \mathrm{C}$ for 18 hours. Mating spots were collected using 360 a Nunc $10 \mu \mathrm{L}$ sterile plastic inoculation loop and resuspended in $500 \mu \mathrm{L}$ of ASW. Then, $100 \mu \mathrm{L}$ of this suspension were spread onto TSB2 plates supplemented with $\mathrm{Cm}$ and incubated at $25^{\circ} \mathrm{C}$ for 48 hours. Finally, the mutant library (totaling 26,662 mutants) was archived in $500 \mu \mathrm{L}$ aliquots with $\mathrm{ASW}$ supplemented with $\mathrm{Cm}$ and $25 \%$ glycerol (v/v), quickly frozen in a dry ice bath for 10 minutes and then stored at $-80^{\circ} \mathrm{C}$ until testing. 
Resistant mutants were selected by challenging the mutant library with high titers of phages. Four aliquots of the mutant library were defrosted, centrifuged by pelleting at 5,000 x $g$ for five minutes, and then washed twice with $2216 \mathrm{MB}$ to remove any residual glycerol. The washed pellets were then resuspended in their original tube with one $\mathrm{mL}$ of fresh $2216 \mathrm{MB}$ supplemented with $\mathrm{Cm}$. C8-WT served as positive control and was treated equivalently, except for the addition of $\mathrm{Cm}$. The washed mutant library and C8-WT control were both diluted 1:10 in $2216 \mathrm{MB}$ and then incubated at room temperature with vigorous shaking $(250 \mathrm{rpm})$ for 1 hour until the cultures reached early exponential phase. To select for phage-resistant mutants, lysates was serially diluted 10 -fold and mixed with the mutant library and C8-WT cultures. Aliquots of host-phage culture were mixed into $750 \mu \mathrm{L}$ of $2216 \mathrm{MB}$ top agar (with and without $\mathrm{Cm}$ as needed) and spread on large $2216 \mathrm{MB}$ bottom agar plates (with and without $\mathrm{Cm}$ as needed) following the soft agar protocol as described previously (54). After incubating at room temperature for 48 hours, $\sim 100$ phage-resistant colonies were selected at random and serially restreaked three times on $2216 \mathrm{MB}$ agar plates (with and without $\mathrm{Cm}$ as needed). Glycerol stocks of each mutant were archived, and all mutants were then re-tested for phage susceptibility. The re380 test was always done with two "orange" and two "purple" phages, one of each always being the original phage used to isolate resistant colonies. In all cases, resistance to one "orange" phage yielded resistance to all "orange" phages and resistance to one "purple" phage yielded resistance to all "purple" phages. Cross resistance to opposite or both phage groups was never seen, further supporting the finding that each group of phages uses a different receptor.

$385 \quad$ Arbitrary PCR (90) was used to map the transposon insertions in resistant strains. Genomic DNA from each phage-resistant mutant was extracted with Lyse-n-Go direct PCR reagent (Thermo Scientific), and one $\mu \mathrm{L}$ of the lysate served as template in arbitrary PCR. This method involved two rounds of PCR amplification (90): in the first round, genomic DNA was amplified with a fully degenerate primer SS9arb2 (Data S8) containing a 5' tail of known390 sequence to be used for specific amplification in the second round of PCR (91), paired with primer Mar4 (Data S8) that binds the end of the transposon TnSC189 (92). Optimized conditions for the first round PCR consisted of the following reagent concentrations and amplification parameters: primers SS9arb2 and Mar4 were at $0.5 \mathrm{mM}$ and $0.2 \mathrm{mM}$, respectively; GoTaq G2 HotStart (Promega) was used with $\mathrm{MgCl}_{2}$ at two $\mathrm{mM}$; initial heating for two minutes at $95^{\circ} \mathrm{C}$, 395 followed by six cycles of 30 seconds at $95^{\circ} \mathrm{C}, 30$ seconds at $30^{\circ} \mathrm{C}$, and 1 minute and 30 seconds at $72^{\circ} \mathrm{C} ; 30$ cycles of 30 seconds at $95^{\circ} \mathrm{C}, 30$ seconds at $55^{\circ} \mathrm{C}$ and one minute and 30 seconds at $72^{\circ} \mathrm{C}$, with a final extension for five minutes at $72^{\circ} \mathrm{C}$. In the second round of PCR amplification, $2.5 \mu \mathrm{L}$ of the first-round PCR product was used as template, combined with a nested primer within the amplified fragment of TnSC189 (Mar4_int2) and primer (Arb3) with sequence 400 identity to the 5' tail of the SS9arb2 (Data S8). For the second round, PCR reagents were used as described above but using both primer concentrations were $0.2 \mathrm{mM}$, and the PCR was run under the following conditions: two minutes at $95^{\circ} \mathrm{C}, 30$ cycles of 30 seconds at $95^{\circ} \mathrm{C}, 30$ seconds at $58^{\circ} \mathrm{C}$ and one minute and 30 seconds at $72^{\circ} \mathrm{C}$, with a final extension time of five minutes at $72^{\circ} \mathrm{C}$. PCR products were verified by electrophoresis, purified by spin-column using QIAquick 405 PCR Purification (Qiagen) and then Sanger-sequenced. Finally, amplicons were trimmed and mapped to the C8-WT genome to identify transposon insertion locations using a custom python script and BLASTn (64). Hits are present in Table S1.

Receptor identification using re-sequencing of spontaneously resistant isolates 
As an independent method to transposon mutagenesis, we re-sequenced spontaneously resistant mutants from co-cultures of "orange" host C8-WT and high titer phages to identify phage receptors. C8-WT was streaked out from glycerol stock onto 2216MB agar plates, inoculated into five $\mathrm{mL}$ of $2216 \mathrm{MB}$ liquid media, gown shaking overnight at room temperature, and plated as a lawn in a soft agar overlay. Five $\mu \mathrm{L}$ drop spots of a phage dilution series were plated on top of the agar, and after 24 hours, resistant colonies that grew in the presence of high phage concentrations were re-streaked three times and archived. For each phage, 10 colonies were archived. Resistant strains were re-streaked and re-tested to verify resistance. The re-test was always done with two "orange" and two "purple" phages, one of each always being the original phage used to isolate resistant colonies just as in the transposon experiments. The results were also consistent with the transposon mutagenesis experiments: In all cases, resistance to one "orange" phage yielded resistance to all "orange" phages and resistance to one "purple" phage yielded resistance to all "purple" phages. Cross resistance to opposite or both phage groups was never seen. Six to seven strains verified in this way were sequenced on an Illumina HiSeq as described in the "Hybrid genome assemblies" section. Reads were trimmed and mapped to the hybrid assembly reference genome using CLC work bench 9. Single nucleotide polymorphisms (SNPs) and indels were identified using a custom pipeline made for CLC work bench 9 and are presented in Table S2. These SNPs were cross-referenced to receptor identification using transposon mutagenesis (see above).

$430 \quad$ Receptor verification using gene deletion or complementation To formally demonstrate the role of a gene predicted to encode a phage receptor, clean deletions were performed using ccdB based suicide vector pSW7848T $(89,93)$. Fragments $(\sim 0.5 \mathrm{kbp})$ flanking the locus to delete were amplified and assembled by PCR, then cloned into pSW7848T using the Gibson Assembly Master Mix (New England Biolabs) according to the manufacturer's 435 protocol. The plasmid was transferred from the E. coli donor strain (u3914) to the recipient vibrio by conjugation as previously described. Selection of the plasmid-borne drug marker on TSB-2

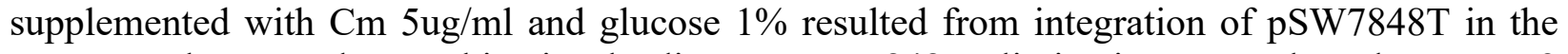
genome. The second recombination leading to pSW7848T elimination was selected on TSB-2 supplemented with arabinose $0.2 \%$. Mutants were screened by PCR using external primers $440 \quad$ flanking the different targets.

Because GspH seemed to be essential, and we could not knock it out, we took the spontaneously resistant mutant with a SNP in gspM and complemented in the WT of the same gene to restore killing. The WT gene was amplified by PCR (see primer details in Data S8) and cloned in Apa1/Xho1 sites of the pMRB plasmid under the control of a constitutive Plac

445 promoter (94).

Distribution of putative receptors across diverse vibrios

To test how diverse receptor genes identified by mutant analysis in $V$. lentus are across other vibrios, we used the species and population designations from Arevalo et al., (37). In cases

450 where a species was composed of multiple populations, we chose to only analyze the population with the most members. We based our identification of core genes on the method described in Arevalo et al. (37). ORFs were identified with Prodigal 2.6 (71) and orthologous genes were clustered using MMseqs2 (60). Core genes for a given population were defined as orthologs which were present in a single copy in all members of that population. We aligned all genes within orthologous clusters using MUSCLE (95) and calculated the amino acid diversity between 
each pair of genes as the number of positions with non-identical amino acids divided by the alignment length. Average pairwise amino acid diversity was obtained by taking the mean diversity across all pairs of genes within an orthologous cluster. The amino acid sequences of each receptor gene were aligned using mafft-linsi (74) and average pairwise amino acid diversity was calculated as described above.

Phylogenetic relationships among receptors identified using genetic approaches were determined across a collection of diverse Vibrio isolates (73). Receptor orthologues were identified using BLASTn (83) with evalue of 1E-8, aligned using MAFFT (74), and final trees were constructed using RAxML (75) with model GTRCAT.

To estimate whether diverse isolates are decorated with similar lipopolysaccharides (LPS), we extracted the genome region bounded by gmp and $g m h D(96)$, which encodes genes for LPS synthesis. We searched the same Vibrio genomes from Arevalo et al. (37), that were used for the determination of known defense genes in Figure S7. When the same set of genes was present, we inferred that the LPS structure was highly similar and may thus bind to similar phage receptors. We only analyzed genomes that had LPS regions that were present on a single contig to avoid ambiguity in presence/absence patterns. Genes were clustered using MMseqs2 (60) at 90\% identity and required to be present in at least 2 genomes. Presence/absence profiles are shown in Figure S18C using hierarchical clustering to order the genomes (rows) and LPS genes (columns). Genes with identical presence absence patterns in Figure S18C have only one tree branch going into the left-most gene (top tree).

\section{$\underline{\text { RNA-Seq analysis of "orange" PDEs }}$}

We diluted 200uL an overnight culture of the WT "orange" host in $200 \mathrm{~mL}$ of fresh media and grew it to mid exponential phase in an Erlenmeyer flask. Then, we added a representative 480 "purple" phage at an $\mathrm{MOI}=10$ or $\mathrm{MOI}=5$. Every 15 minutes, for a total of 11 timepoints, we archived 500uL of the culture in Qiagen RNAProtect, and then placed on dry ice. Once the time course was collected, we extracted the total RNA for sequencing using a Qiagen RNEasy extraction kit, following the manufacture's instructions. RNA was sequenced at the BioMicro Center at MIT. By mapping the transcripts to the host genome, we were able to identify regions that appeared to be transcribed together. Reads were filtered and trimmed using trimgalore version 0.5.0 with the following options: trim_galore -e 0.05 --length 70 --max_n 1 --error_rate 0.05 --stringency 1 --clip_R1 1 --three_prime_clip_R1 1. Reads originating from the virus and from ribosomal RNAs were removed using bbslit version 38.18. Reads were mapped to the region using bbwrap version 38.18 with the strandedcov $=$ t option to later manually identify 490 putative transcriptional units. Gene expression was estimated using Kallisto version 0.46.2 with the following options: kallisto quant --single -1 200 -s 20. Both replicates (MOI=10 and MOI=5) were consistent in expression for the 3 PDEs analyzed (Fig. S11).

\section{Phage defense element knockouts using two-step allelic exchange}

495 To test whether putative PDEs were responsible for phage resistance in C8-WT, we knocked out large portions of each PDE containing genes annotated as being related to phage defense (Fig. $2 \mathrm{~A}$ ), in all possible combinations. We found it was possible to knock out nearly all of PDE1 $(93.5 \%)$, but had to leave in part of the element's $y f b r$ gene as it replaces the host's $y f b r$ gene upon insertion. For PDE2, we found that knocking out the entire element was not possible in a 500 single step, likely because of the toxicity effects of deleting the entire putative TA/Abi systems at once. We therefore proceeded to make a partial deletion $(58.8 \%)$ from the helicase/toxin gene to 
the 5 ' end of the element, leaving the putative antitoxin intact, along with other genes predicted to play roles in insertion/mobilization of the element (integrases, transposases, and recombinases) as indicated by structure and function annotations using HHpred (97), InterProScan5 (62), and Phyre2 (56) (Fig. 2A, Data S3). Noting PDE3 also has a similar putative TA system, we followed the same approach as for PDE2 and made a partial deletion $(68.8 \%)$ from the helicase/toxin gene to the 5 ' end of the element. The details of the approach are summarized in Figure 2A.

Site-directed mutagenesis was used for all deletions and cloning was carried out using the New England Biolabs Gibson Assembly Master Mix according to the manufacturer's protocol. Fragments upstream and downstream of the portion of the element to be deleted were separately PCR-amplified using primers specified in Data S7. A third PCR reaction was carried out to amplify the backbone of the plasmid pSW7848T (93) (Data S7) with primer pairs pSW_F\&R (Data S7). These amplicons were cut with Dpn1 (two hours at $37^{\circ} \mathrm{C}$ ) to inactivate the plasmid 515 template before setting up the Gibson assembly reaction. In all cases, PCR products were verified by gel electrophoresis, purified by spin-column as described above, and DNA concentration was determined using Nanodrop 2000 (Thermo Scientific). Subsequently, 0.03 pmol of each vector was assembled with 0.07 pmol of its specific downstream and upstream DNA fragments at $50^{\circ} \mathrm{C}$ for 60 minutes. After completion of this reaction, DNA was desalted by dialysis on a $0.0025 \mu \mathrm{M}$ filter (Millipore) before electroporation into $E$. coli $\Pi 3813$, which was used as a plasmid host for cloning (89). Finally, the plasmid DNA was purified, verified by Sanger sequencing, and electroporated into E. coli $\beta 3914$ to be uses as a plasmid host for conjugation (89) (Data S7).

Conjugation was carried out in a mating spot as described above for the transposon mutagenesis but with some modifications: donor to recipient ratio was changed to $3: 1$, the mating media was altered to MMB-2 (TSB supplemented with $2 \% \mathrm{NaCl}$ plus dapA), and the mating spot was incubated at $30^{\circ} \mathrm{C}$. Counter-selection of $\Delta$ dapA donor was performed by plating on TSB2 agar plates without dapA but supplemented with $\mathrm{Cm}$ and glucose $1 \%(\mathrm{w} / \mathrm{v})$. Antibioticresistant colonies are due to the integration of the entire plasmid $\left(\mathrm{Cm}^{\mathrm{R}}\right)$ in the chromosome by a single crossover. Colonies were picked, re-grown in liquid media (TSB2) supplemented with Cm and glucose $1 \%(\mathrm{w} / \mathrm{v})$ to late logarithmic phase and spread on BD Bacto TSB without Dextrose plates supplemented with $2 \% \mathrm{NaCl}(\mathrm{w} / \mathrm{v})$ and $0.2 \%$ arabinose. To verify deletions in the single PDE mutants $\triangle \mathrm{PDE} 1, \triangle \mathrm{PDE} 2$ and $\triangle \mathrm{PDE} 3$ (Data S7) PCR products generated using primers flanking externally the different regions targeted ( $\triangle \mathrm{PDE} 1 / \mathrm{F} \& \mathrm{R} ; \Delta \mathrm{PDE} 2 / \mathrm{F} \& \mathrm{R}$ and $\Delta \mathrm{PDE} 3 / \mathrm{F} \& \mathrm{R})$ (Data S7) were sequenced by Sanger. This procedure was also used to construct double $(\Delta \triangle \mathrm{PDE} 12 ; \Delta \triangle \mathrm{PDE} 13 ; \Delta \Delta \mathrm{PDE} 23)$ and triple mutants $(\Delta \Delta \Delta \mathrm{PDE} 123)$ but using a single or double mutant as final recipient during the conjugation step (Data $\mathrm{S} 7$ ).

Single phage defense gene characterization using transposon mutagenesis or complementation

540 To identify the specific genes involved in phage defense and further characterize the PDEs discovered in this work, we took advantage of our double mutants to scrutinize the third remaining PDE. For example, using the $\triangle \triangle$ PDE23 knockout, we used integration of a suicide vector by single recombination to inactivate genes hypothesized to underlie phage defense (using the same protocol described under "receptor verification"). Using this approach, we identified 545 that inactivating the restriction gene of the restriction modification system on PDE1 was necessary to yield loss of defense. The analogous approach was also successful in determining the genes involved in phage defense on PDE2. We found four genes within a module on the 5' 
end of the element was each involved in defense: unannotated genes 10N26155C8 ORF_0_597, putative GTPase 10N26155C8 ORF_0_598, putative GTPase 10N26155C8 ORF_0_599, unannotated gene 10N26155C8 ORF_0_600, and unannotated gene 10N26155C8 ORF_0_601 with DUF4231.

However, this method was unsuccessful for determining the genes involved in PDE3. While we used the same approach to inactivate 11 different genes, which we hypothesized to be involved in defense based on superficial annotations, no genes responsible for defense to "purple" phages were identified. Instead, we took a transcriptomics approach (described under "RNA-Seq of "orange" PDEs"). This allowed identification of 14 regions that appeared to be transcribed together. All regions were cloned by Gibson assembly in a pMRB plasmid previously depleted from the Plac promoter by a PCR inside out. These regions carry 2-3 genes under the control of native promoters. Each construct was transferred into the C8 triple mutant $\Delta \triangle \triangle \mathrm{PDE} 123$ that is fully sensitive to the "purple" phage. Although six regions were not able to be cloned into E. coli, possibly due to toxicity, and thus could not be further analyzed (R1, R2, R4, R5, R11, R13), this approach led us to identify Region 3 as sufficient for defense. This region contains only two predicted full length ORFs: ORF 806, which is a short unannotated gene, and ORF 807 which is annotated as having a conserved domain of unknown function 565 (DUF2971). However, through inactivation, we did not see any phenotype for ORF 806, leading us to conclude ORF 807 was most likely the gene of interest.

\section{Phage susceptibility assay for whole PDE deletion mutants}

To test the susceptibility of the "orange" PDE deletion mutants to "purple" phages, we challenged each mutant with representative "purple" phage 1.281.O in agar overlays (Fig. 2B) and in liquid culture (Fig. S13). The plate-based findings were corroborated in liquid, although the subtle phenotypic alterations for single PDE knockouts required careful plating to observe. These experiments were also repeated to test the mutants against all "orange" and "purple" phages as well (Fig. S12).

$575 \quad$ For the mutant testing in agar overlays, we used the same protocol outlined in the "Host range assays at varying phage concentrations" section above, with one additional step: after the plaques were imaged, we re-streaked the phages from the highest concentration drop spot onto fresh bacterial (host or mutant) lawns to test for phage propagation (Fig. 2C). We imaged the agar overlay plates using a flatbed scanner (Epson Perfection V800 Photo Scanner - Product No.

580 B11B22320) and captured using VueScan Software by Hamrick as before. However, for the entire panel of data in figures $2 \mathrm{~B}$ and $2 \mathrm{C}$ alone, we also optimized visualization by manipulating the images in Adobe Photoshop. Specifically, applying "Auto contrast" on all images. No other images throughout the text were manipulated.

For the liquid assay, we streaked out each mutant onto $2216 \mathrm{MB}$ agar plates, allowed 48

585 hours for large colonies to form, and inoculated each mutant into $3 \mathrm{~mL}$ of $2216 \mathrm{MB}$ in triplicate. After growing the cultures shaking at $25^{\circ} \mathrm{C}$ for four hours, we normalized one $\mathrm{mL}$ of the culture to an $\mathrm{OD}_{600}$ of 0.3 , diluted it 100x into a final volume of five $\mathrm{mL}$, and aliquoted $200 \mu \mathrm{L}$ into 12 wells each of a 96-well clear bottom Micro-titer plate (Falcon). A Tecan Microplate Reader with Spark software was used to maintain the cultures shaking at $25^{\circ} \mathrm{C}$, monitoring $\mathrm{OD}_{600}$ every 15 $590 \quad$ minutes. Once OD 600 reached 0.3, "purple" phage 1.281.O was added at different concentrations to reach the desired multiplicities of infection (Fig. S13), after which the cultures were returned to the plate reader for the remainder of a 24-hour run. This experiment was run in triplicate for 
each biological replicate. It was run with two mutants, C8-WT, and "purple" host 10N28654F7 each time until all mutants had been tested.

Quantification of PDEs within clonal hosts across the Nahant Time Series

Samples for total community DNA were described previously (36). Briefly, samples were collected on each of the 94 days in the time series. Upon return to the lab, water samples were filtered using a peristaltic pump through a $0.2 \mathrm{um}$ Sterivex filter into a secondary $4 \mathrm{~L}$ collection bottle. To extract total community DNA, filters were carefully cut and placed in two $\mathrm{mL}$ tubes with $0.25 \mathrm{~g}$ of sterile $0.1 \mathrm{~mm}$ zirconium beads and $750 \mu \mathrm{L}$ of Qiagen Cell Lysis Solution, and then run on a Beadbeater at $5000 \mathrm{rpm}$ for 60 seconds. Samples were then incubation at $80{ }^{\circ} \mathrm{C}$ for five minutes and cooled to room temperature, and RNA was digested by adding four $\mu \mathrm{L}$ RNAse A (four $\mathrm{mg} / \mathrm{mL}$ ), mixed by inverting the tubes, and incubated at $37^{\circ} \mathrm{C}$ for 30 minutes. DNA was purified by adding $250 \mu \mathrm{L}$ of Qiagen Protein Precipitation Solution, mixed by vortexing for 20 seconds, incubated on ice for five minutes and centrifuged at 13,000 rpm for five minutes. Supernatant was transferred to a new tube and centrifuged again to ensure removal of all precipitates. Subsequently, $750 \mu \mathrm{L}$ of isopropanol were added to $750 \mu \mathrm{L}$ of supernatant to precipitate DNA and incubated at $-20^{\circ} \mathrm{C}$ overnight. DNA was recovered by centrifugation at $13,000 \mathrm{rpm}$ for five minutes and washed with $700 \mu \mathrm{L}$ of $70 \%$ ethanol. Finally, the DNA was dried and resuspended in $100 \mu \mathrm{L}$ of Qiagen brand DNA hydration solution.

To quantify PDEs carried within a specific host genomic background, we carried out digital droplet PCR (ddPCR) on the time series total DNA samples. Custom ddPCR assays were designed using Primer3Plus with the "Probe" server settings activated to target three inserted phage defense elements, two unique to the "orange" group (PDE2 and PDE3) and one unique to the "purple" group (PDE4). The amplified product always spanned the insertion site of the element, while the probe targeted either the insertion site or the element of interest depending on design constraints of the assay. Complementary probes (FAM/HEX) were used to allow for assay multiplexing and final assays were ordered through Bio-Rad. For the PDE2 and PDE4 multiplexed assay, $20 \mu \mathrm{L}$ of a $22 \mu \mathrm{L}$ reaction containing $11 \mu \mathrm{L}$ ddPCR Supermix for Probes (No dUTP) \#1863024 master mix, 1.1 $\mu \mathrm{L}$ PDE4 Assay, 1.1 $\mu \mathrm{L}$ PDE2 Assay, $1.1 \mu \mathrm{L}$ BsmI restriction enzyme (NEB R0134L) diluted in CutSmart buffer, and $7.7 \mu \mathrm{L}$ of 10-fold diluted sample were prepared for analysis and run on the standard ddPCR thermocycler program using a Tm of $60^{\circ} \mathrm{C}$. For the PDE3 assay, $20 \mu \mathrm{L}$ of a $24 \mu \mathrm{L}$ reaction was run using standard recommendations from Bio-Rad. All primers and probes are listed in Data S7. 
A

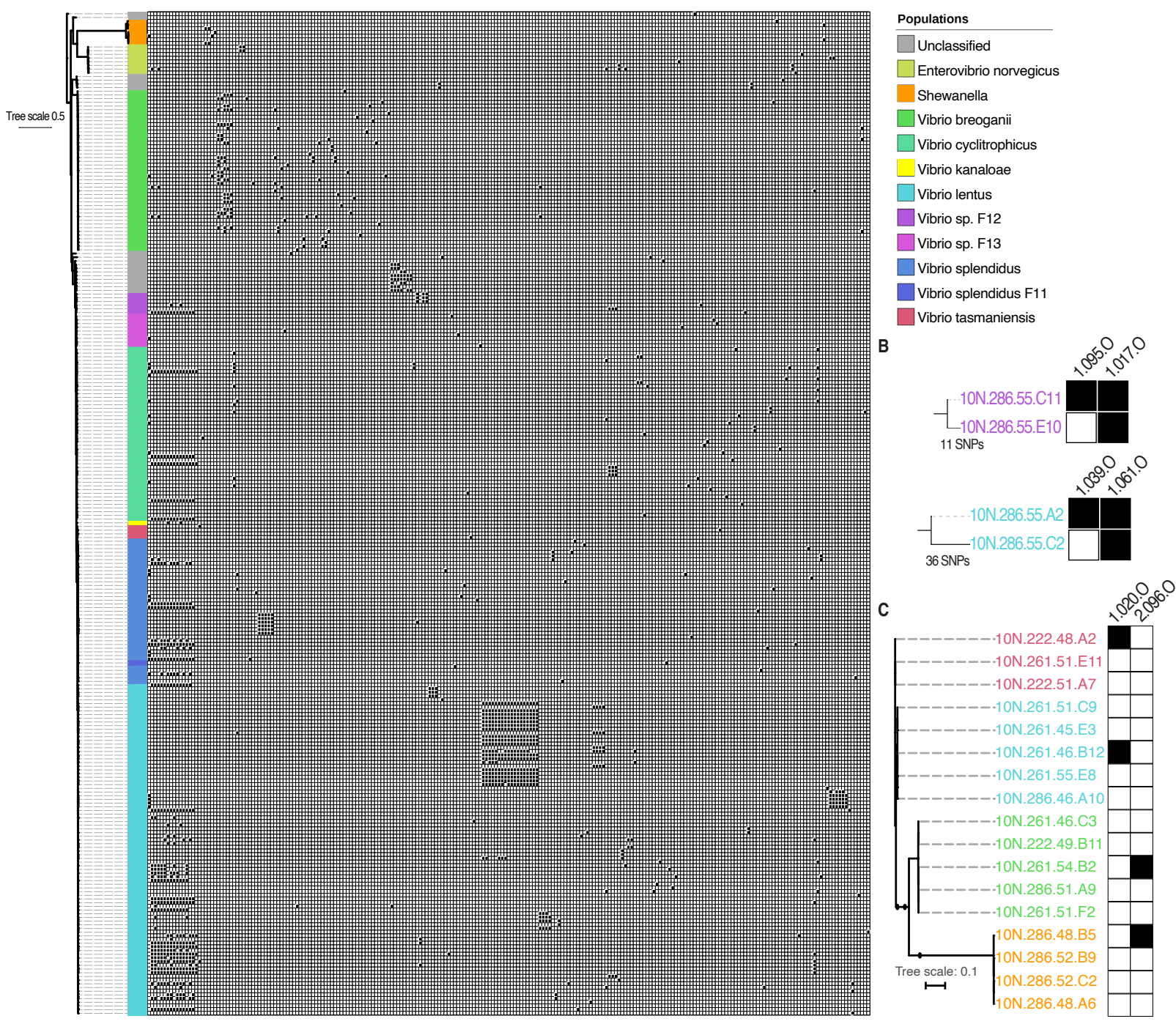

Fig. S1.

Phage host-range established using an exhaustive cross-test matrix. (A) Full matrix with rows depicting bacterial hosts organized by the phylogeny of their ribosomal protein and $h s p 60$ gene sequences (proxy for core genome), and columns depicting phages ordered by protein similarity identity [modified from Figure 2 in (34)]. (B) Closest bacterial relatives differing in phage sensitivity profiles can be distinguished by only few SNPs across their entire core genomes. Trees represent full genome alignments, phage identification codes written above columns, black boxes indicate positive infection determined by plaque assay. (C) Broad host-range phages, defined as host ranges spanning different species, remain strain-specific within different species. Phylogenetic tree constructed using same alignment of core genes as in A, and infection representation analogous to that in B. Source data available in Kauffman et al. 2021 (35). 
A

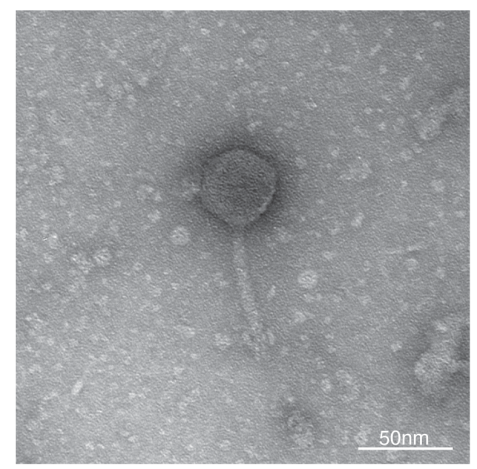

c

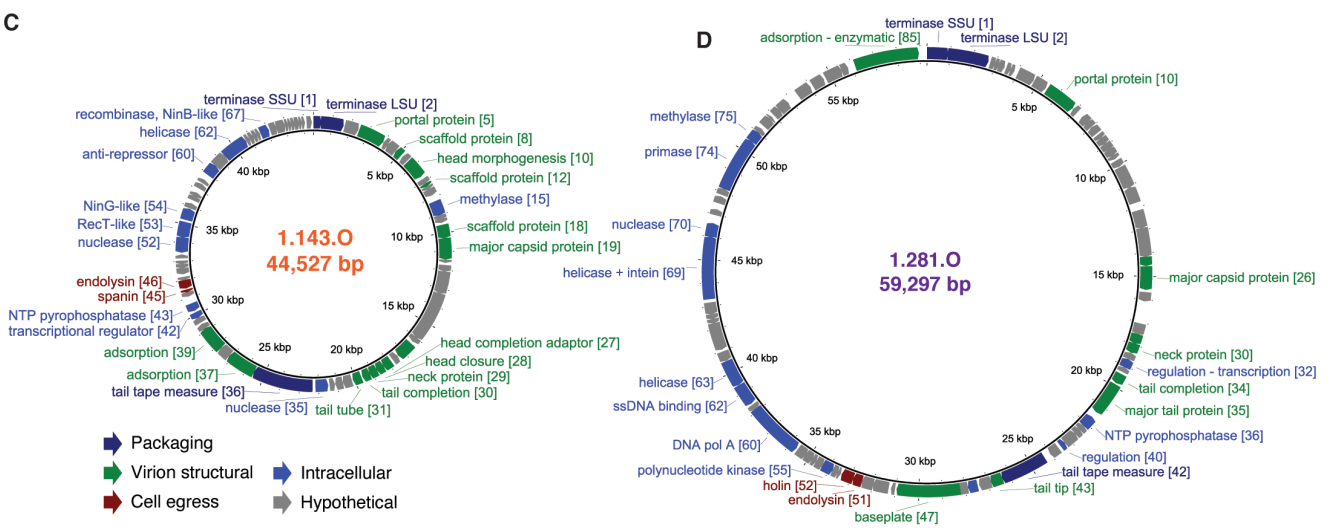

E

640

\section{Fig. S2.}

B

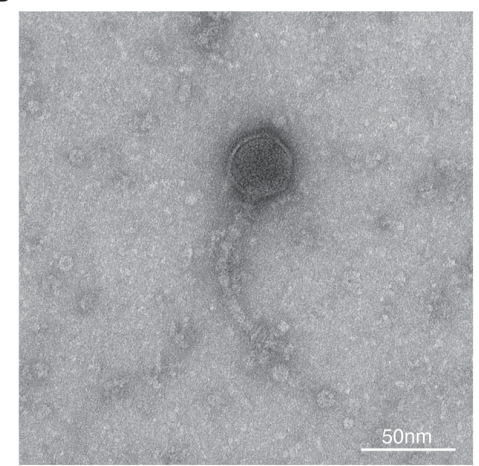

D

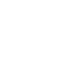

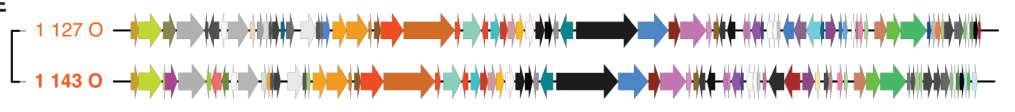

F

"Orange" and "purple" phages represent divergent groups of siphoviruses. (A, B) Electron microscopy of phages representative of "orange" (MG592514.1) and "purple" (MG592649.1) groups suggests that both are siphoviridae, with long non-contractile tails. (C, D) Genome characterization of phages representative of "orange" and "purple" groups, respectively, shows that they differ in size by nearly $15 \mathrm{kbp}$; numbers adjacent to annotations reflect GenBank locus tag. (E, F) Clustering and alignment of phage genomes show that they represent two distinct genus-level groupings. While within each group gene synteny and content are conserved, no gene clusters are shared between groups. Colors are chosen at random to indicate distinct protein sequence clusters (clustered using MMseqs2 v. 2.23394 under default settings). Accessions of phages shown: 1.127.O, MG592501.1; 1.143.O, MG592514.1; 1.267.O, MG592632.1; 1.270.A, MG592636.1; 1.281.O, MG592649.1; 1.269.O, MG592635.1; 1.283.A, MG592651.1; 1.171.O, MG592538.1. 
A

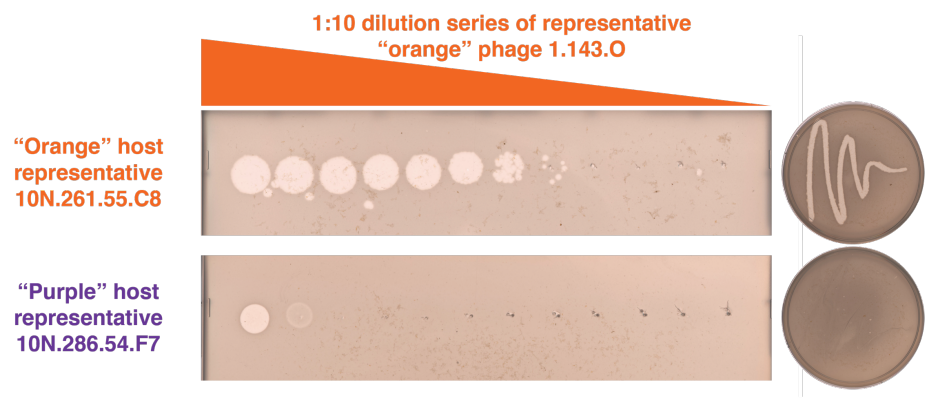

B

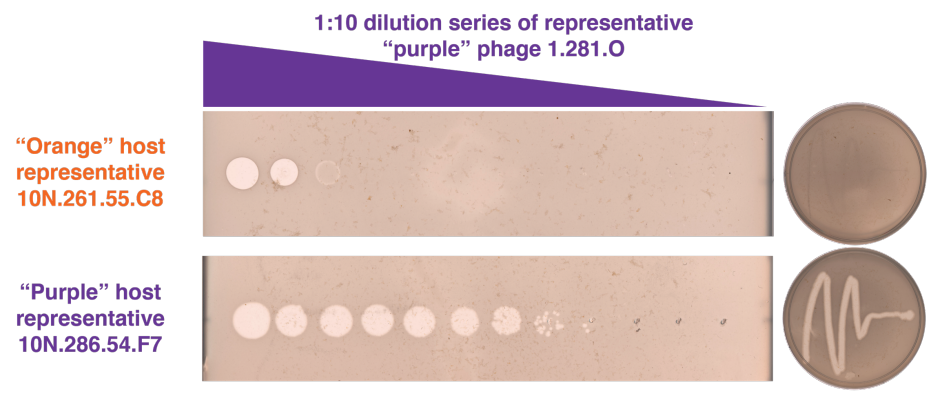

Fig. S3.

Efficiency of plating assay demonstrating effect of differing phage concentrations on host

660 killing. At high concentrations, phage can lyse non-hosts but without production of viable progeny ("lysis from without") indicating that phage can attach and enter the cell, but that replication is prevented internally. (A) "Orange" and "Purple" host lawns challenged with serial dilutions of "orange" phage followed by re-streak propagation test on the right. (B) "Orange" and "Purple" host lawns challenged with serial dilutions of "purple" phage followed by re-streak propagation test on the right. 


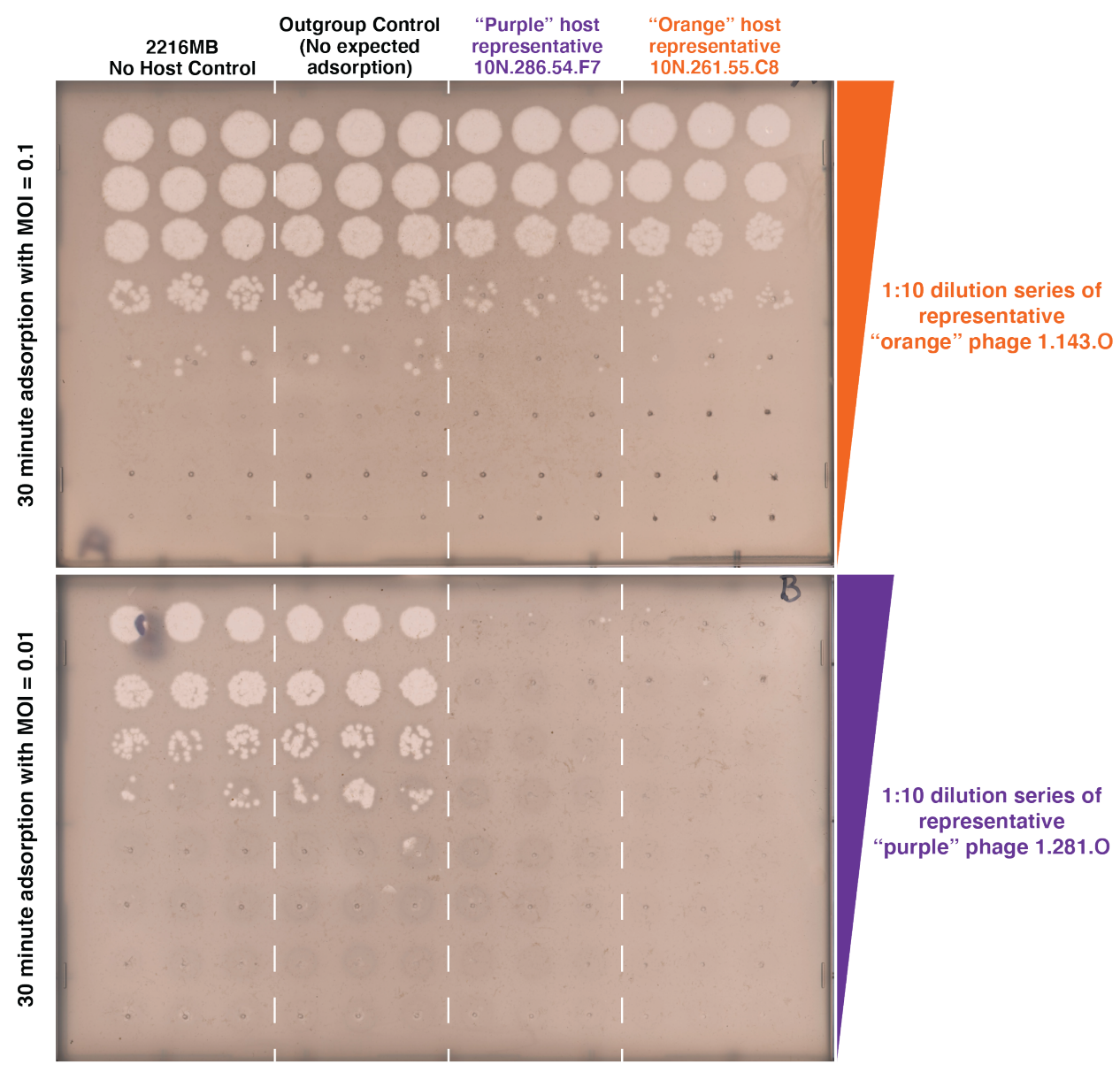

Fig. S4.

Phage adsorption assay showing that phage can adsorb to both "orange" and "purple" strains irrespective of whether those bacterial strains can serve as hosts for viable phage production. After allowing a fixed concentration of phages to adsorb to different bacterial strains, free phages that remained unattached were plated with sensitive hosts to quantify adsorption as the difference to no-host controls (see methods). Both "orange" and "purple" phages were found to adsorb to "orange" and "purple" hosts, but not to an outgroup control. In the top panel, "orange" phage 1.143.O shows the same adsorption phenotype to both "orange" host 10N26155C8 and "purple" 675 host 10N28654F7: the number of free phages decreased by ten-fold. In the bottom panel, "purple" phage 1.281.O shows the same adsorption phenotype to both "orange" host 10N26155C8 and "purple" host 10N28654F7, attaching with full efficiency. In both cases, no attachment is observed for a Vibrio outgroup host $(10 \mathrm{~N} 26149 \mathrm{C} 11)$ as indicated by the same level of phages as in no host controls. Adsorption experiment done with biological and technical triplicates of the represented "orange" and "purple" phages and hosts in this figure. 
A

General Secretion Pathway in V. lentus clones

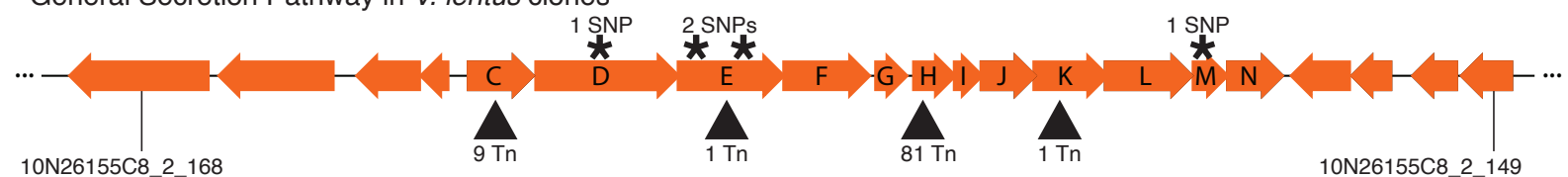

Lipopolysaccharide synthesis pathway in $V$. lentus clones

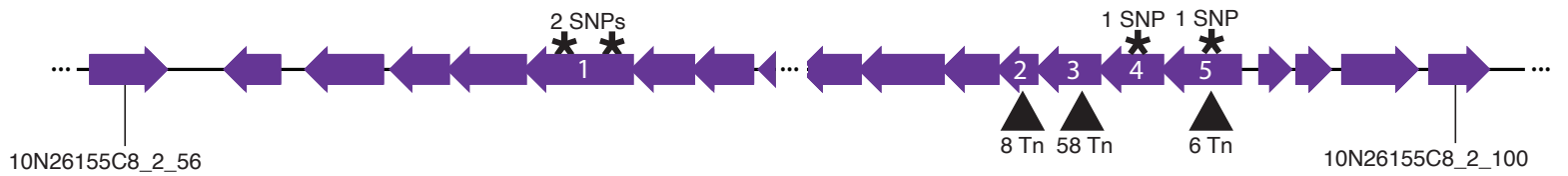

Sodium transport pathway in $V$. lentus clones

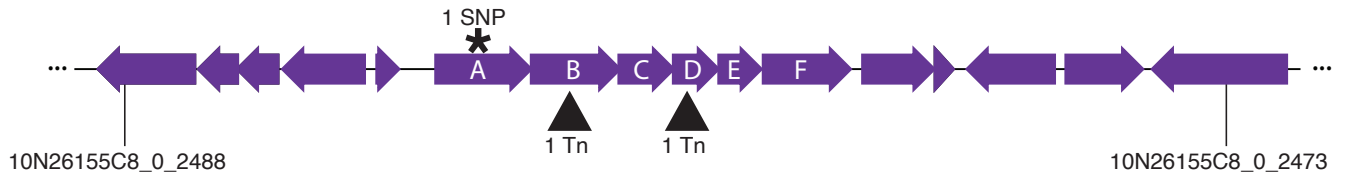

B

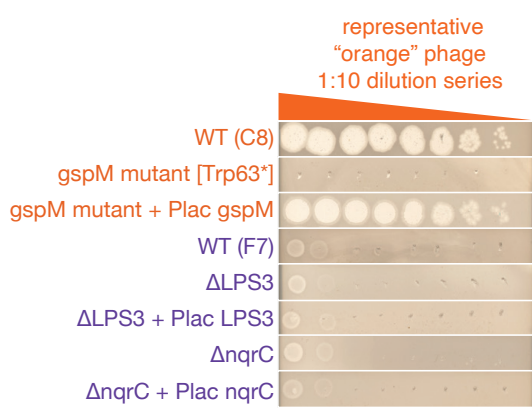

C

representative "purple" phage $1: 10$ dilution series

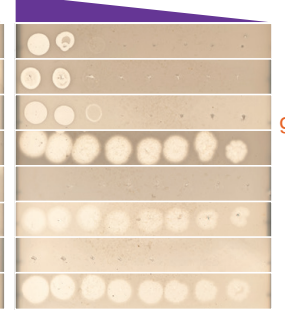

C Tree Scale 0.011

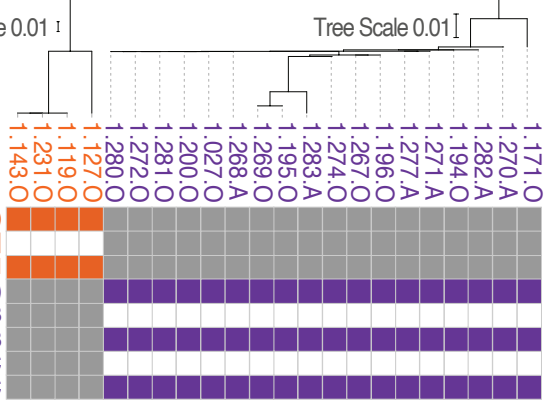

Entry + No Replication

No interaction

Fig. S5. Receptor identification using molecular genetics and experimental evolution. (A) Gene diagrams of loci associated with each receptor in representative "orange" strain 10N26155C8. Transposon insertions depicted with black triangles and SNPs in spontaneously resistant clones indicated with *. (B) Representative lawns of each strain with either SNP, deletion, or complementation challenged with a dilution series of a representative "orange" and "purple" phage. (C) Graphical summary of all-by-all testing of receptor mutants with all phages tested in triplicate (example in $\mathrm{B}$, summarized in $\mathrm{C}$ ). 

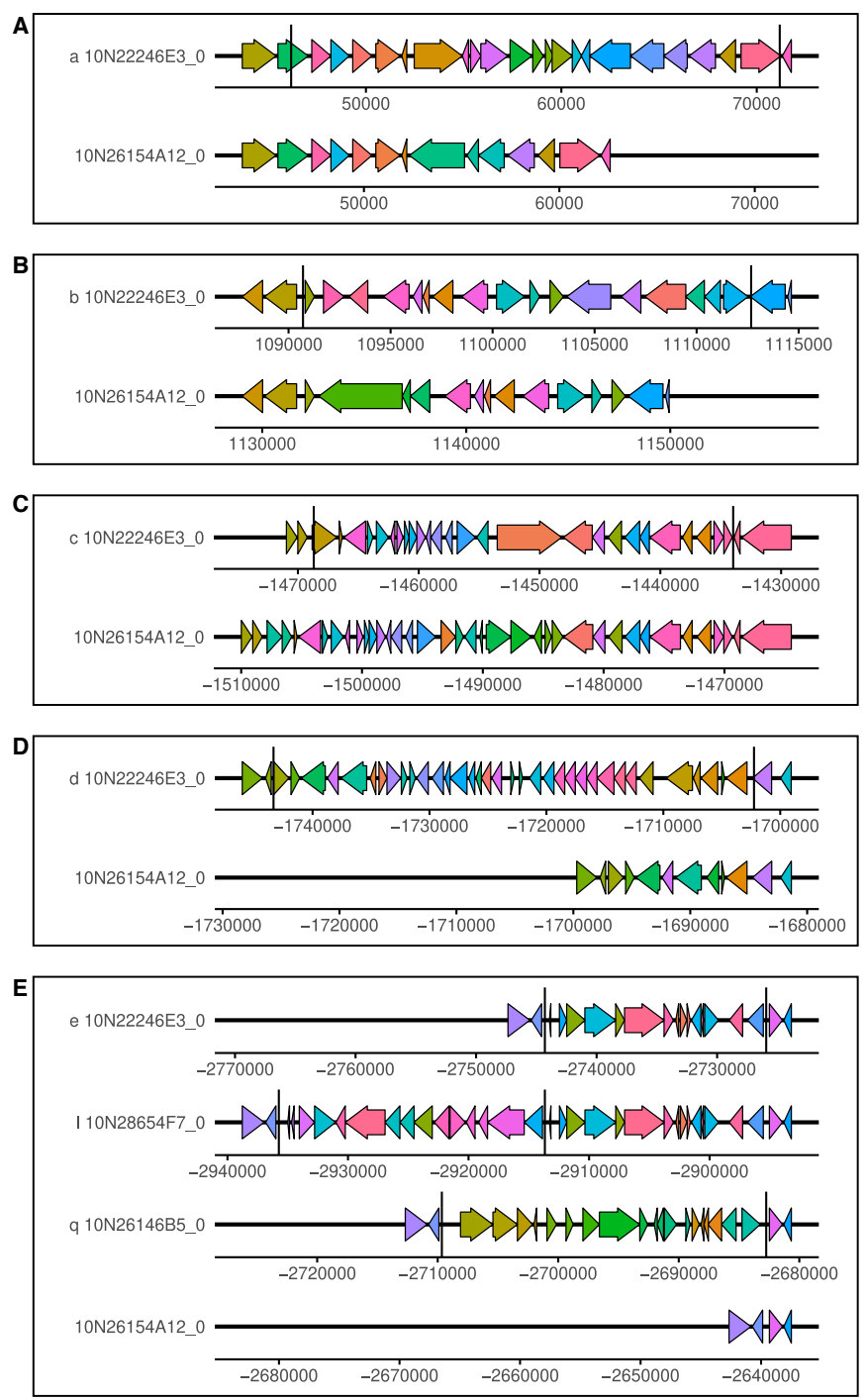

695 Fig. S6. (Part 1) Diagrams of all phage defense elements (PDEs) detected in the 23 V. lentus strains as well as examples of genomes from which PDEs are absent. PDE boundaries as determined by the k-mer-based pipeline are denoted by vertical black lines. Letters match those in Fig. 1B and Data S3. Genes are colored with arbitrary colors indicating $>80 \%$ sequence similarity within each figure panel. Negative genomic coordinates indicate that the region is 700 backwards relative to the reference fasta file used to search. The double star $(* *)$ in I shows an example of diversity within the same PDE among different strains. R and S show putative PDEs that are the entirety of a small contig in the only genome where they were found and therefore the genomic context is unknown/not applicable. 

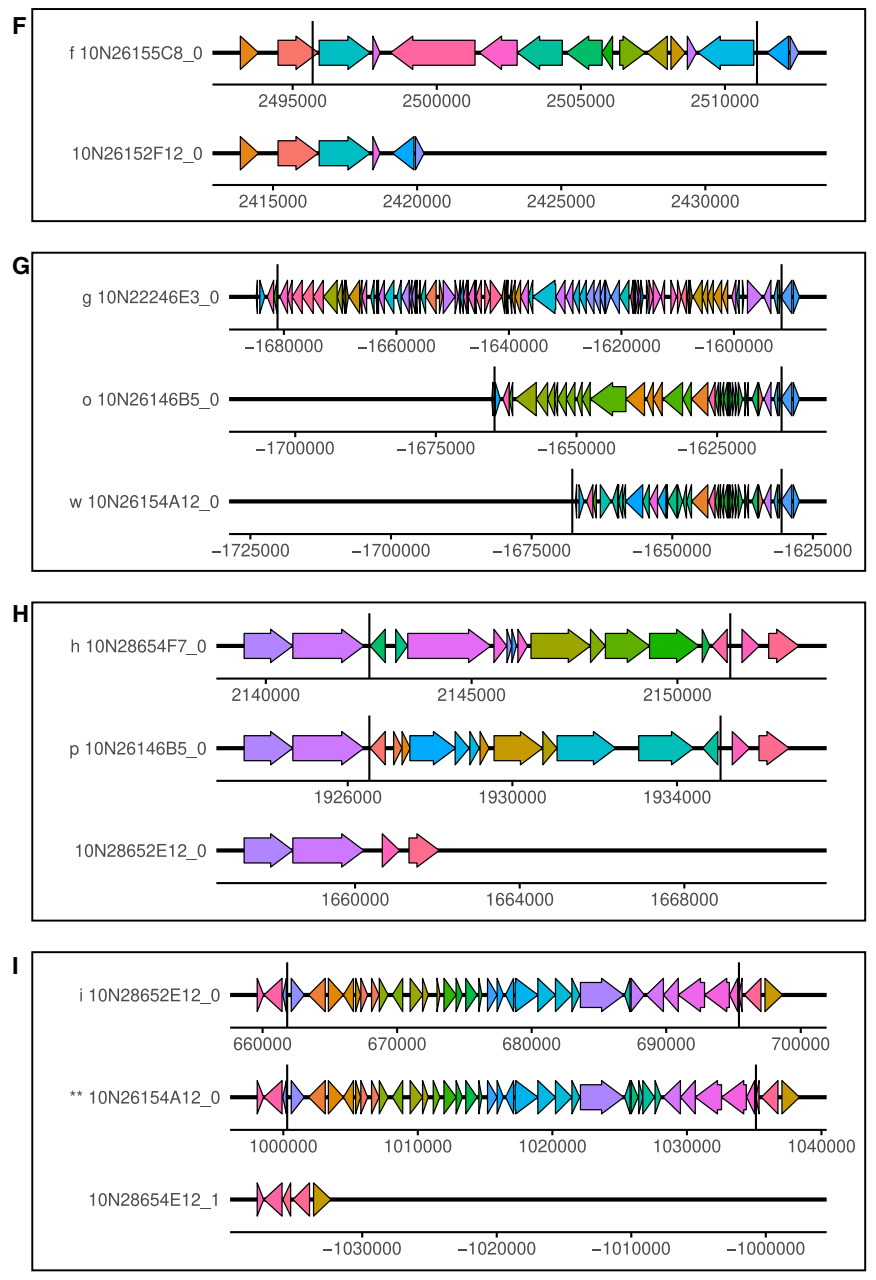

Fig. S6 (Part 2) Diagrams of all phage defense elements (PDEs) detected in the $23 \mathrm{~V}$. lentus strains as well as examples of genomes from which PDEs are absent. PDE boundaries as determined by the k-mer-based pipeline are denoted by vertical black lines. Letters match those in Fig. 1B and Data S3. Genes are colored with arbitrary colors indicating $>80 \%$ sequence similarity within each figure panel. Negative genomic coordinates indicate that the region is backwards relative to the reference fasta file used to search. The double star $(* *)$ in I shows an example of diversity within the same PDE among different strains. $\mathrm{R}$ and S show putative PDEs that are the entirety of a small contig in the only genome where they were found and therefore the genomic context is unknown/not applicable. 

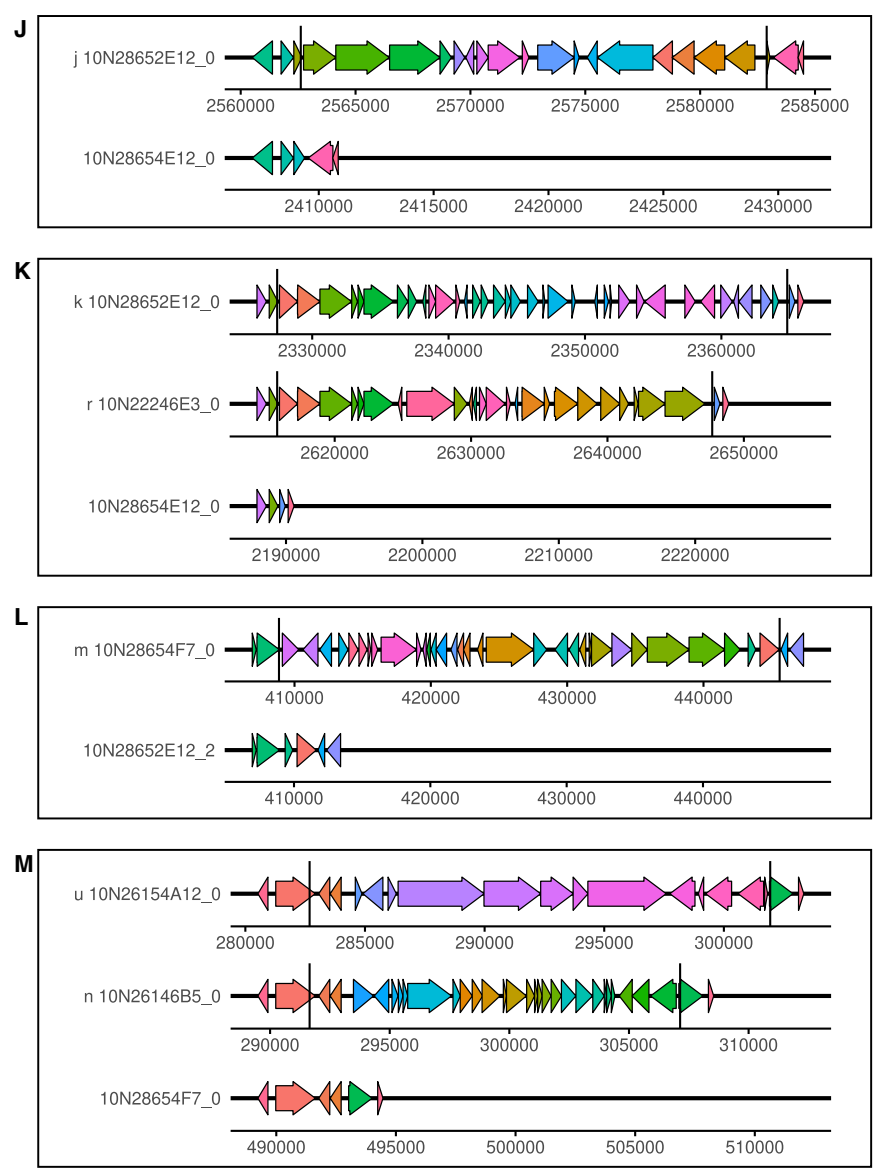

Fig. S6 (Part 3) Diagrams of all phage defense elements (PDEs) detected in the $23 \mathrm{~V}$. lentus strains as well as examples of genomes from which PDEs are absent. PDE boundaries as 725 determined by the k-mer-based pipeline are denoted by vertical black lines. Letters match those in Fig.1B and Data S3. Genes are colored with arbitrary colors indicating $>80 \%$ sequence similarity within each figure panel. Negative genomic coordinates indicate that the region is backwards relative to the reference fasta file used to search. The double star $(* *)$ in I shows an example of diversity within the same PDE among different strains. R and S show putative PDEs that are the entirety of a small contig in the only genome where they were found and therefore the genomic context is unknown/not applicable. 

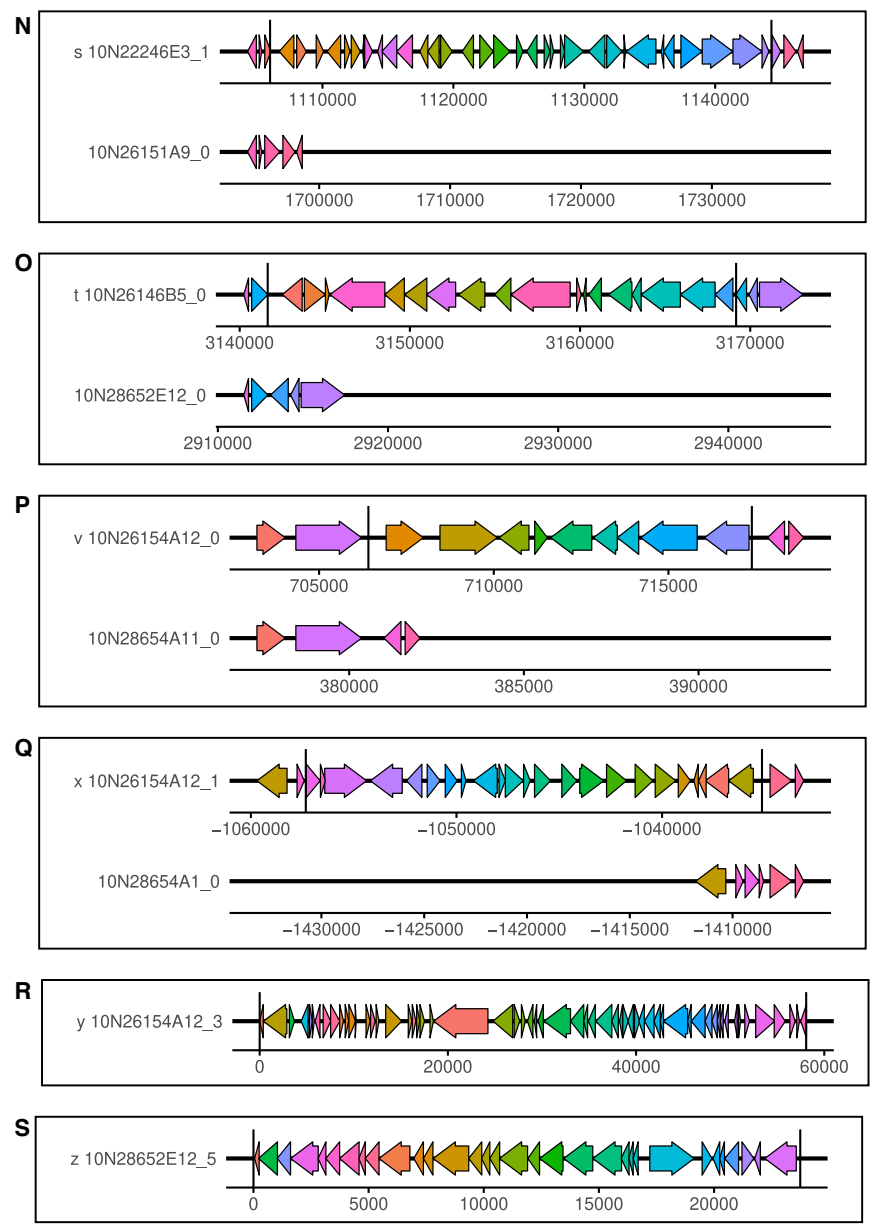

Fig. S6 (Part 4) Diagrams of all phage defense elements (PDEs) detected in the $23 \mathrm{~V}$. lentus strains as well as examples of genomes from which PDEs are absent. PDE boundaries as determined by the k-mer-based pipeline are denoted by vertical black lines. Letters match those

740 in Fig. 1B and Data S3. Genes are colored with arbitrary colors indicating $>80 \%$ sequence similarity within each figure panel. Negative genomic coordinates indicate that the region is backwards relative to the reference fasta file used to search. The double star (**) in I shows an example of diversity within the same PDE among different strains. $\mathrm{R}$ and $\mathrm{S}$ show putative PDEs that are the entirety of a small contig in the only genome where they were found and therefore the genomic context is unknown/not applicable. 


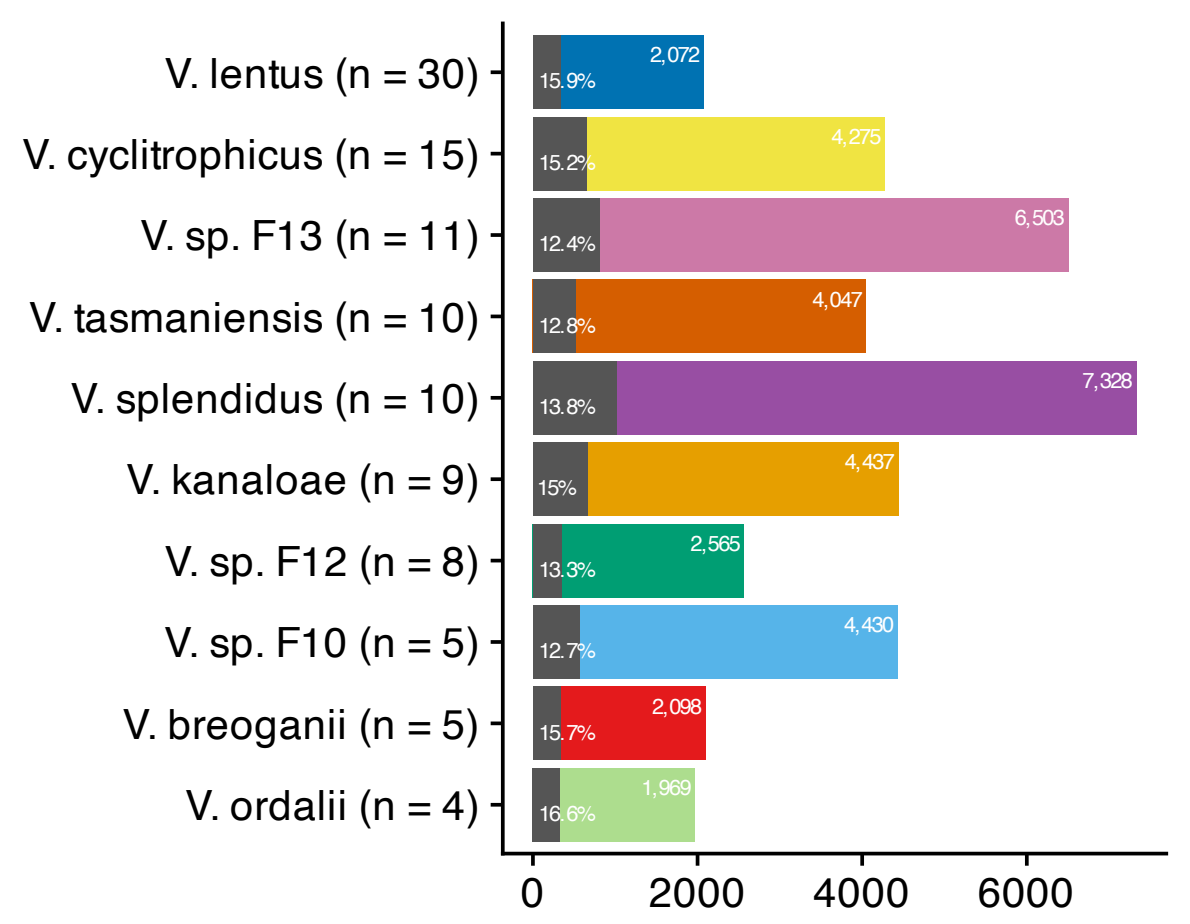

Flexible genes with \% defense hits

Fig. S7. Total number of flexible genes and proportion of known phage defense genes in diverse Vibrio species represented in different colors $(\mathrm{n}=$ number of genomes analyzed). Between 12$770 \quad 17 \%$ (grey bars) of the flexible genes of ten different species, represented as populations defined as gene flow clusters (37), can be attributed to known phage defense genes. 


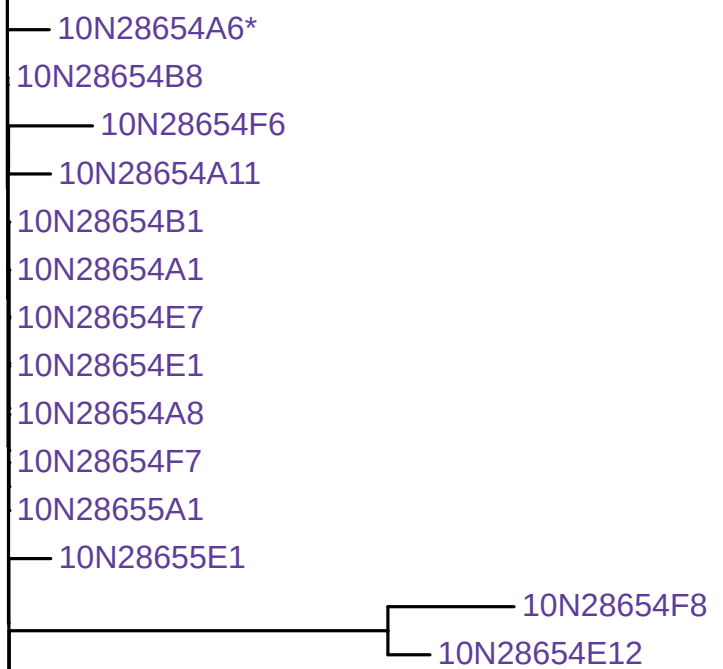

Fig. S8. Unrooted maximum likelihood tree for core genomes of all the nineteen "purple" and 785 "orange" host clones. The strain chosen by the Parsnp program as a reference is indicated by *. 43 SNPs were identified in the total alignment and 14 SNPs differentiate the "orange" and "purple" subsets (see Table S3 for full list). 


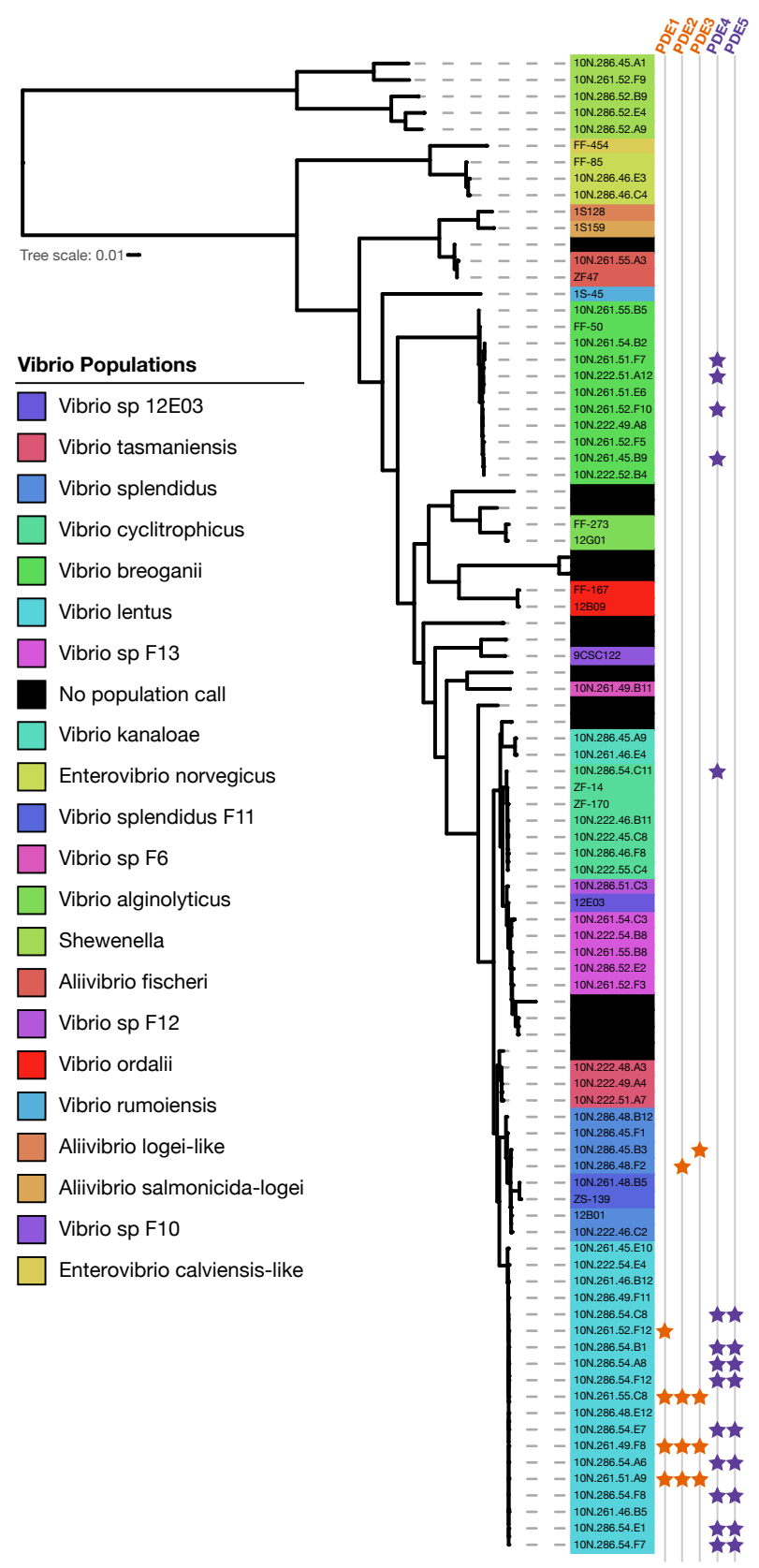

$790 \quad$ Fig. S9. Presence of the same phage defense elements ( $>95 \%$ nucleotide identity over $>90 \%$ of the total element length) in divergent genomic backgrounds suggests their movement via horizontal gene transfer. Pruned tree from Figure S1 depicting the phylogeny of ribosomal protein and hsp60 gene sequences (proxy for core genome) of each Vibrio host. 


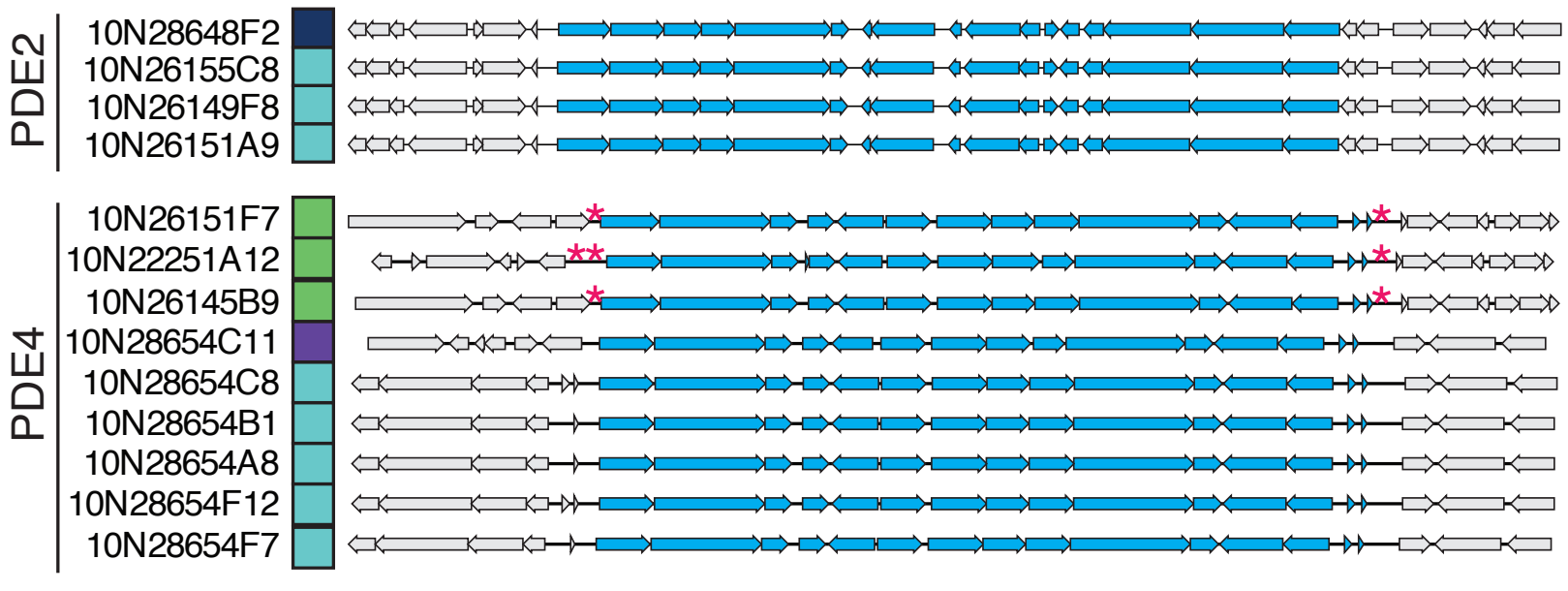

\section{$\square$ Vibrio lentus $\square$ Vibrio splendidus $\square$ Vibrio cyclotrophicus $\square$ Vibrio breoganii \\ $\Rightarrow$ PDE core genes $\quad \Longrightarrow$ Flanking genes * 1-2 SNPs in insertion site}

Fig. S10. Genomic context of horizontally transferred PDEs. PDEs. Specific PDEs for "orange" and "purple" phages are found in closely and distantly related Vibrio, sometimes in different genomic locations, but always at conserved insertion sites with the exception of a few SNPs (indicated with *). PDE2 and PDE4 in different hosts shown as examples from Fig. S9. Lower quality genomes with contig breaks at PDE insertion sites were excluded. 


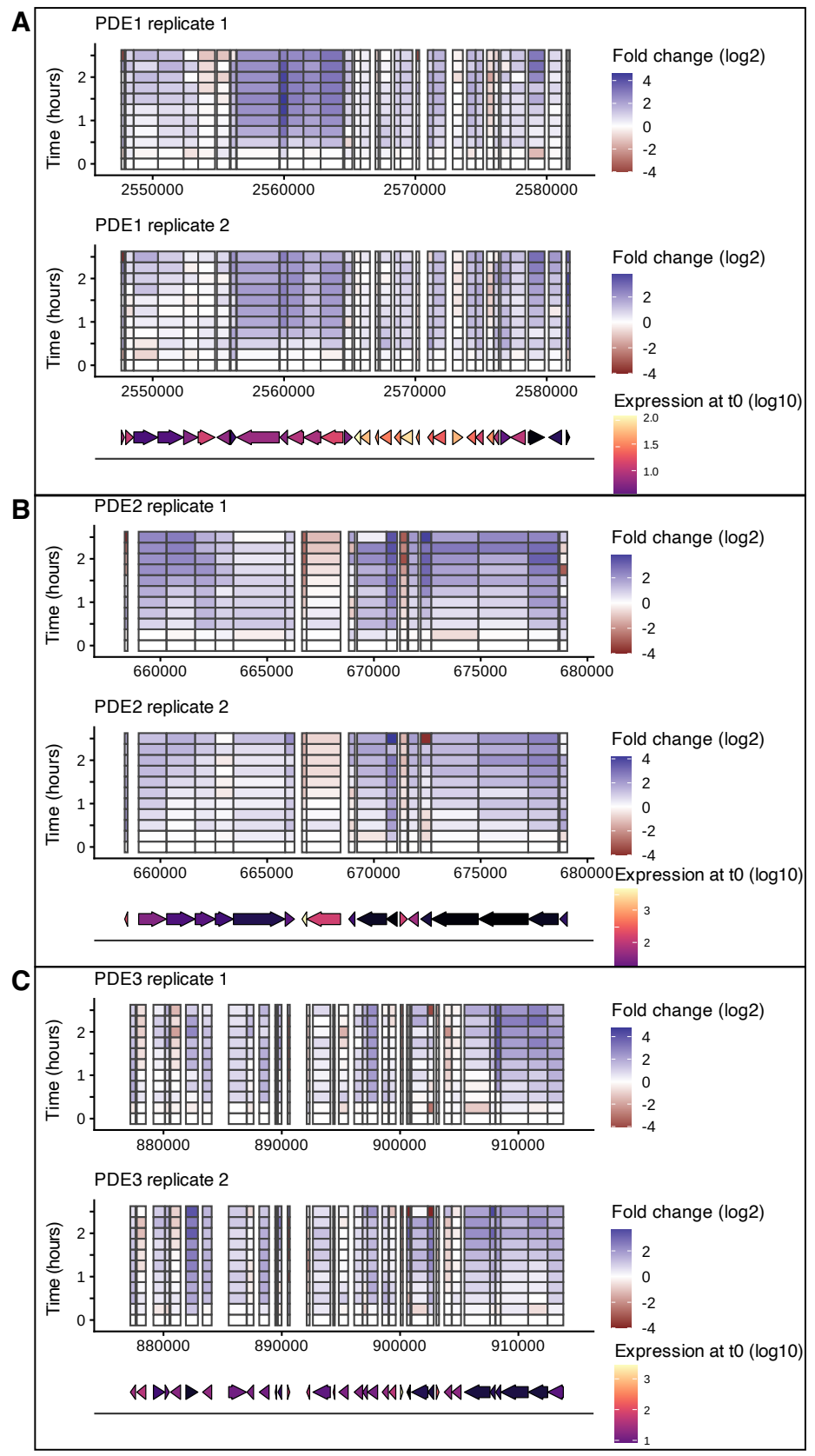

Fig. S11. RNA-seq experiment conducted over a time course of infection of "purple" phage 1.281 .0 on "orange" host $10 \mathrm{~N} 26155 \mathrm{C} 8$ shows changes in gene activity across the 3 PDEs. Boxes indicate transcribed genes and contiguous boxes indicate transcriptional units, colored by fold change in expression compared to time zero (mid exponential phase expression without phage). Gene diagrams for each PDE [(A) PDE1, (B) PDE2, (C) PDE3] are shown below the transcriptomic data summary and colored by the expression at mid exponential phase prior to phage addition $(\mathrm{t}=0)$. Experiment conducted with two replicates. 
A

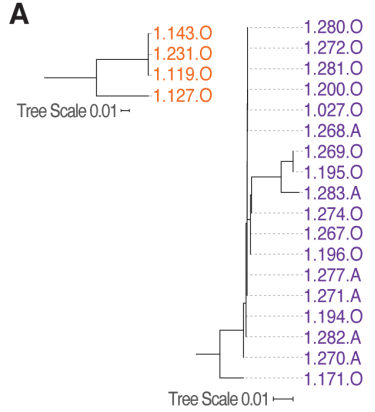

inhibition with plaque propagation

inhibition without plaque propagation
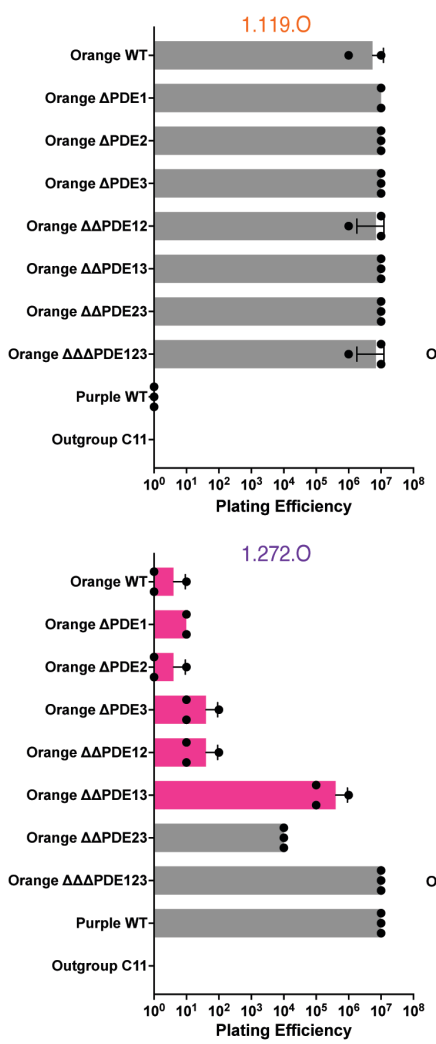

B
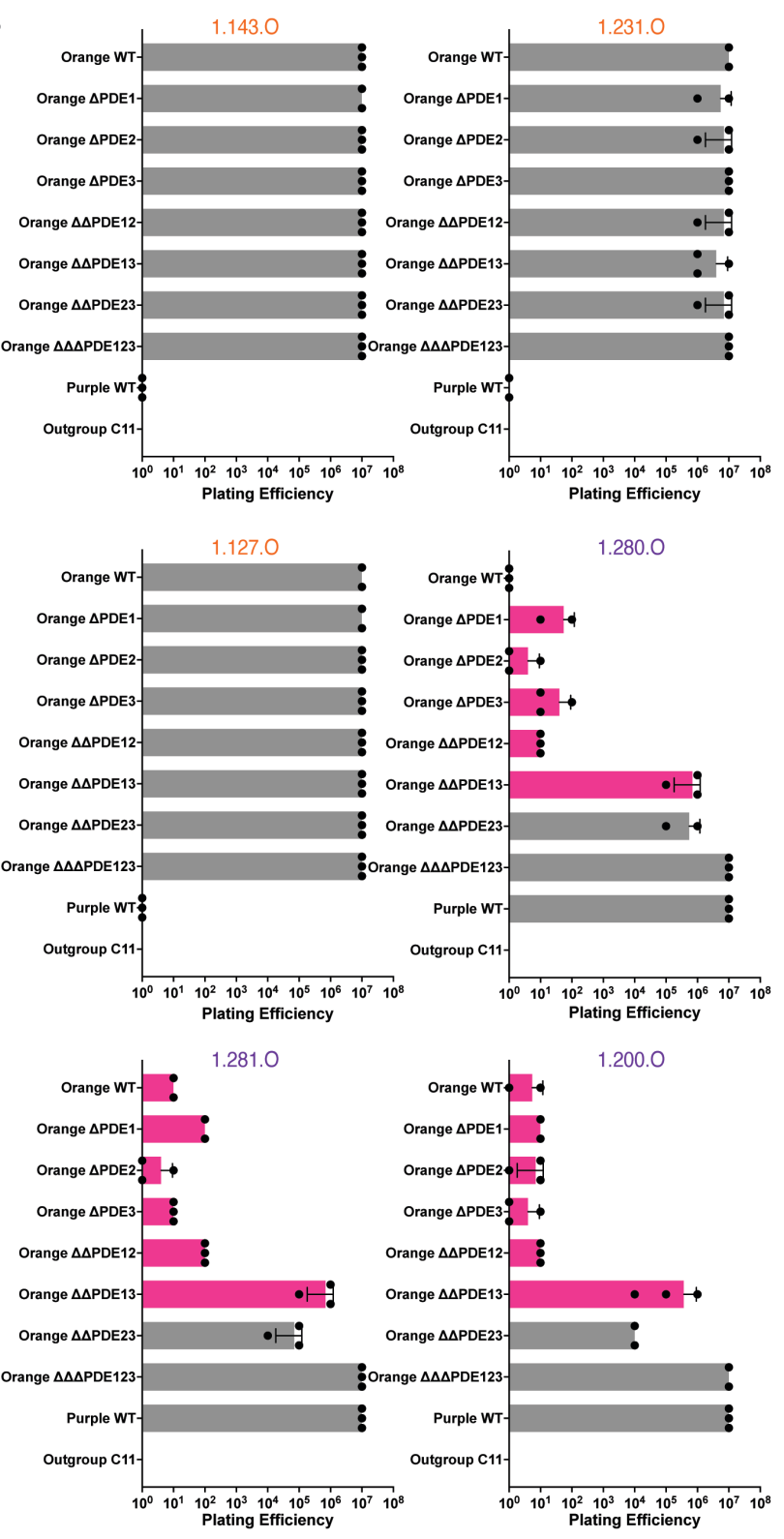

Fig. S12 (Part 1). Efficiency of plating (EOP) assay for all PDE knockouts with all "orange" and "purple" phages. (A) Phage whole genome trees (repeated from Fig. 1A). (B) EOP plating assays for each phage. Pink bars indicate inhibition without plaque propagation and grey bars indicate killing with viable phage production. Triplicates shown as data points and error bars represent standard deviation. 
A

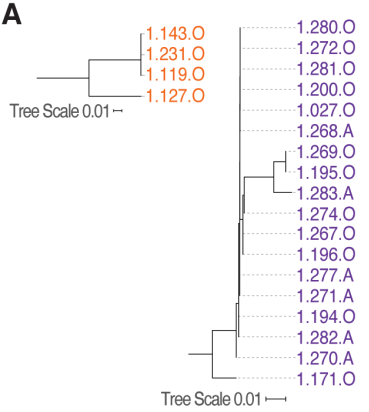

inhibition with plaque propagation

inhibition without plaque propagation
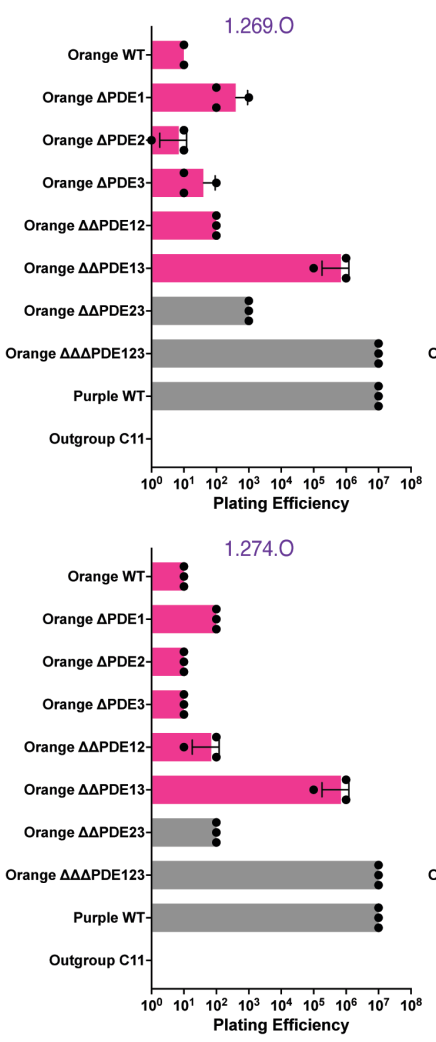

B
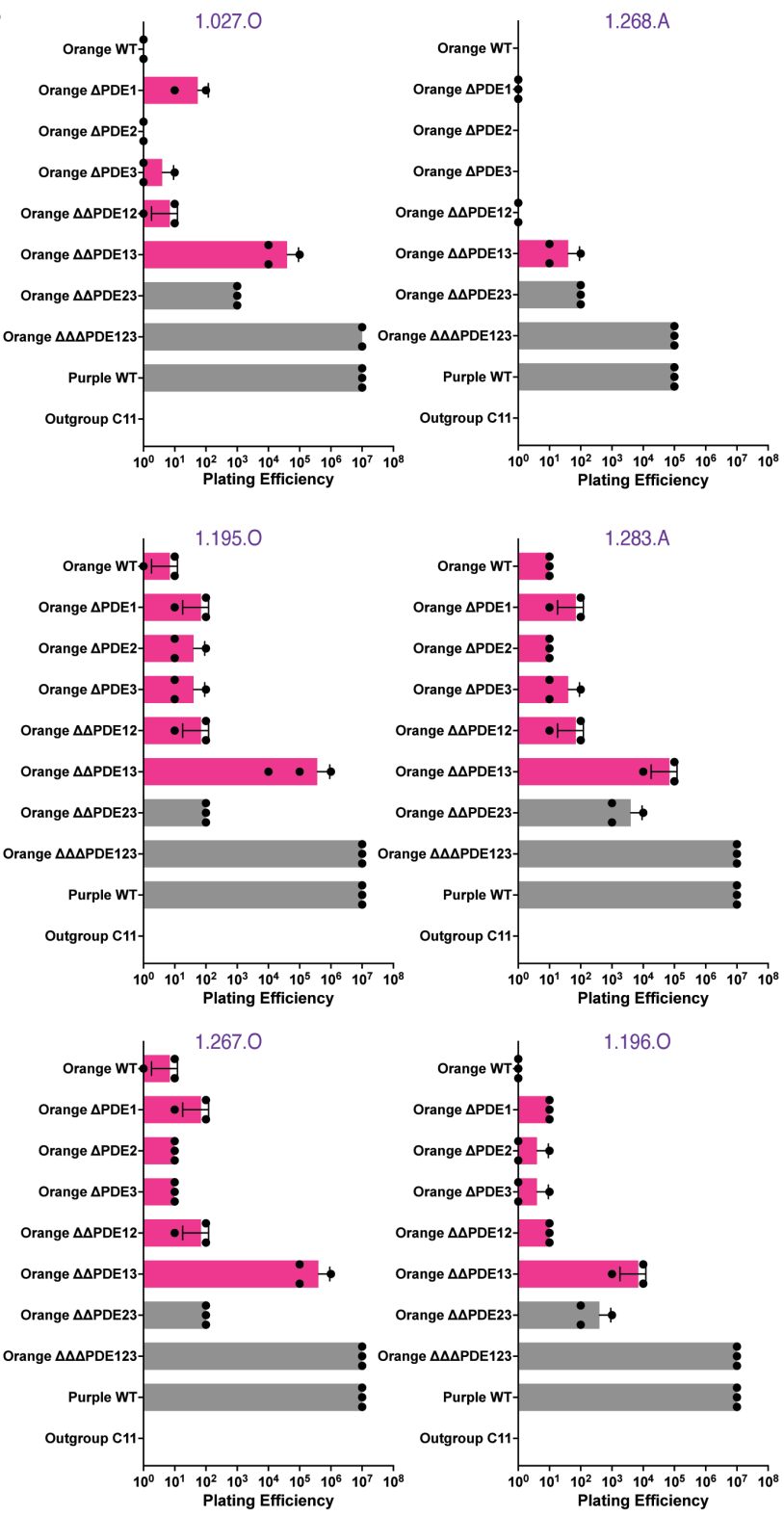

Fig. S12 (Part 2). Efficiency of plating (EOP) assay for all PDE knockouts with all "orange" and "purple" phages. (A) Phage whole genome trees (repeated from Fig. 1A). (B) EOP plating assays for each phage. Pink bars indicate inhibition without plaque propagation and grey bars indicate killing with viable phage production. Triplicates shown as data points and error bars represent standard deviation. 
A

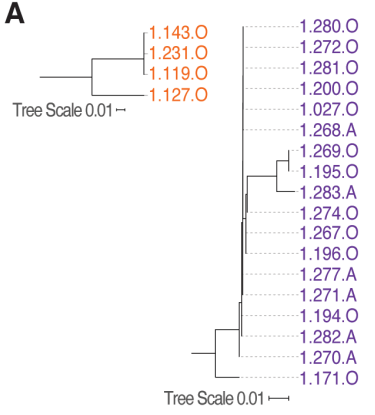

inhibition with plaque propagation

inhibition without plaque propagation
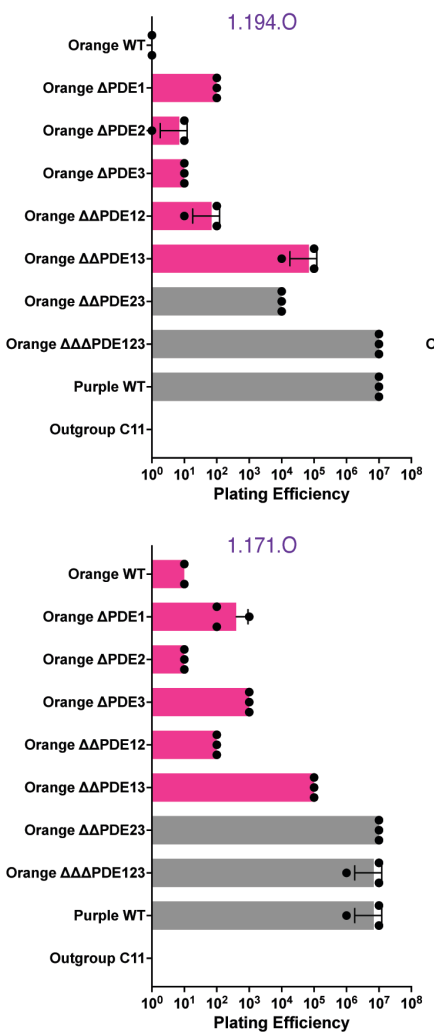

B
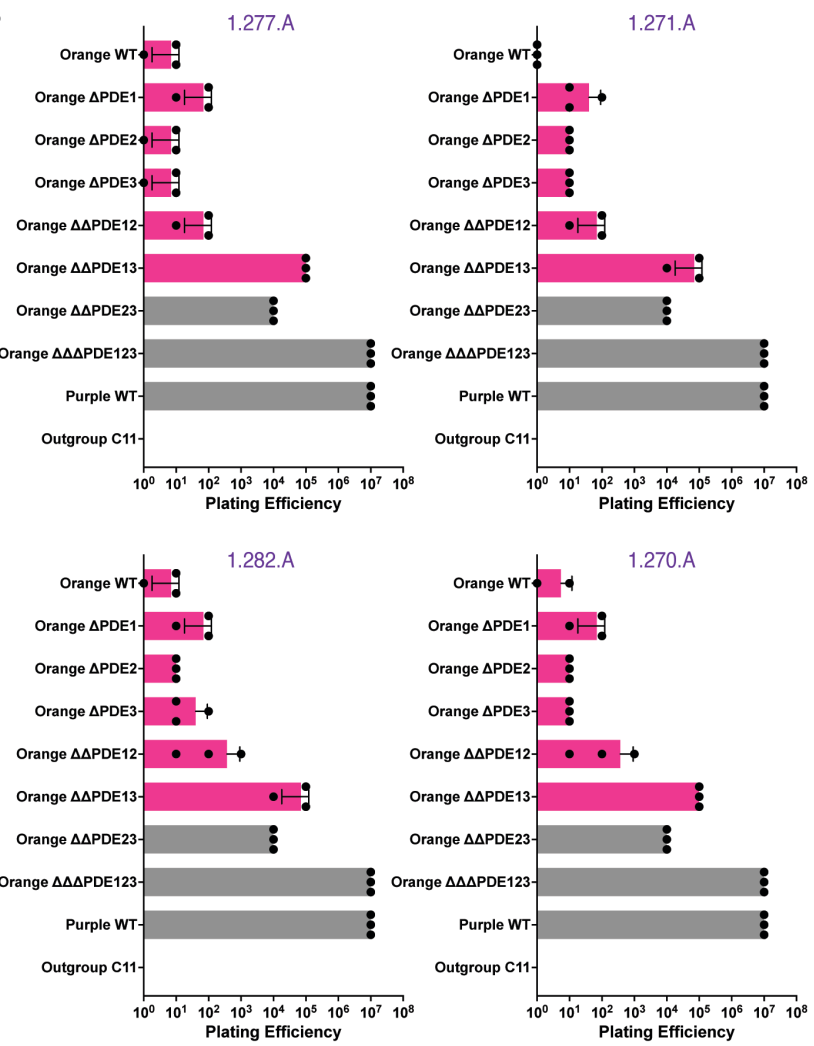

840

Fig. S12 (Part 3). Efficiency of plating (EOP) assay for all PDE knockouts with all "orange" and "purple" phages. (A) Phage whole genome trees (repeated from Fig. 1A). (B) EOP plating assays for each phage. Pink bars indicate inhibition without plaque propagation and grey bars indicate killing with viable phage production. Triplicates shown as data points and error bars represent standard deviation. 


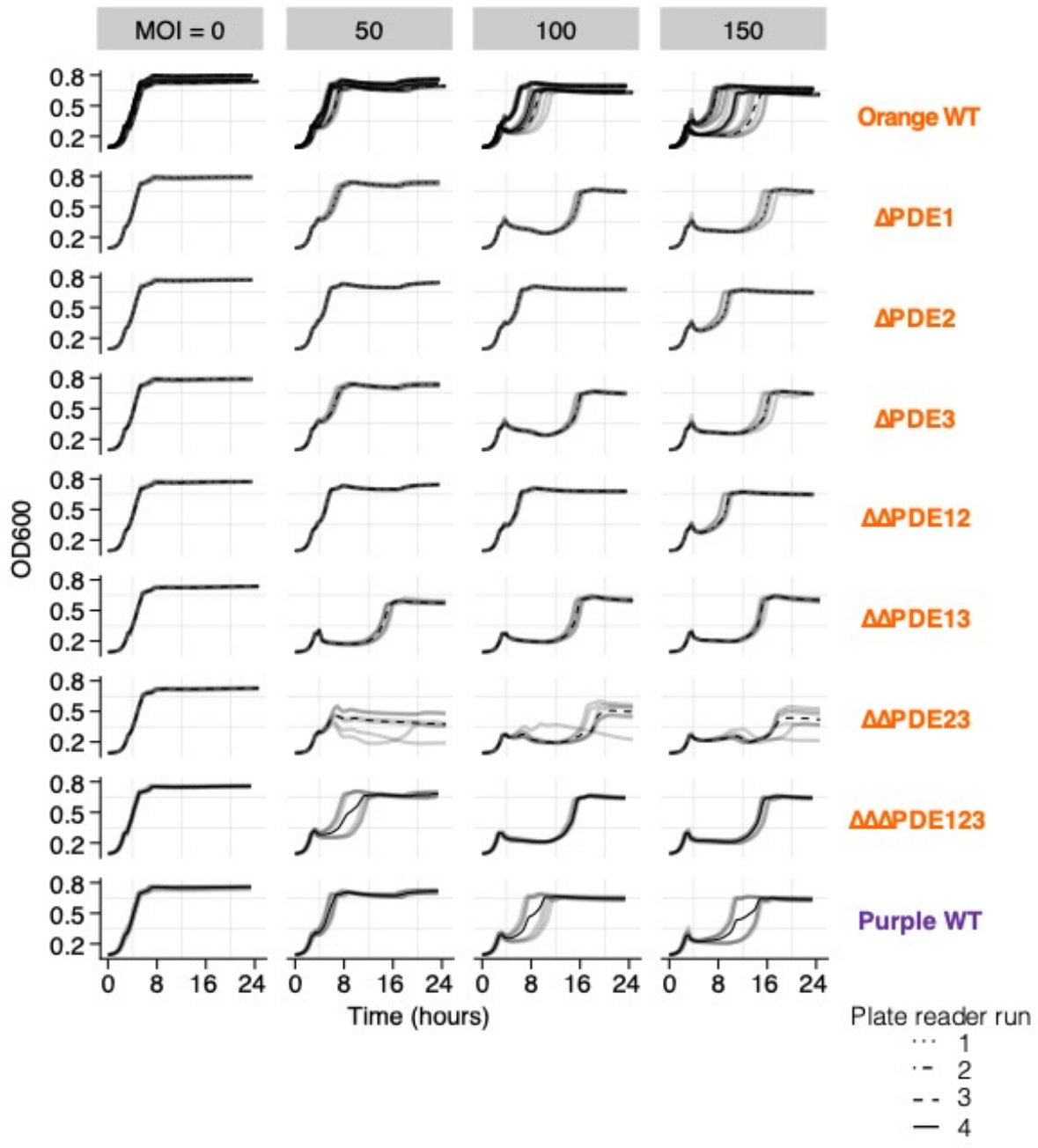

850

Fig. S13. PDE deletions and phage susceptibility testing in liquid. Growth curves of each combination of knockouts grown to mid-exponential phase and then challenged with "purple" phage 1.281.O at varying concentrations. Each experiment consists of three biological replicates, each done in triplicate. High MOIs were used to ensure observance of the "lysis from without" 855 phenotype. 
A

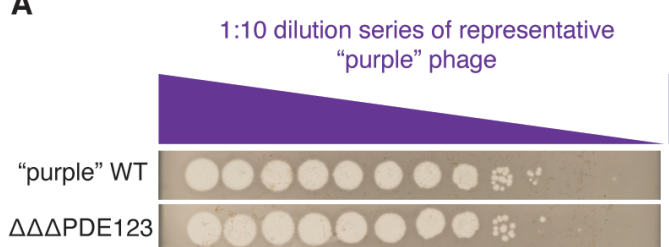

B

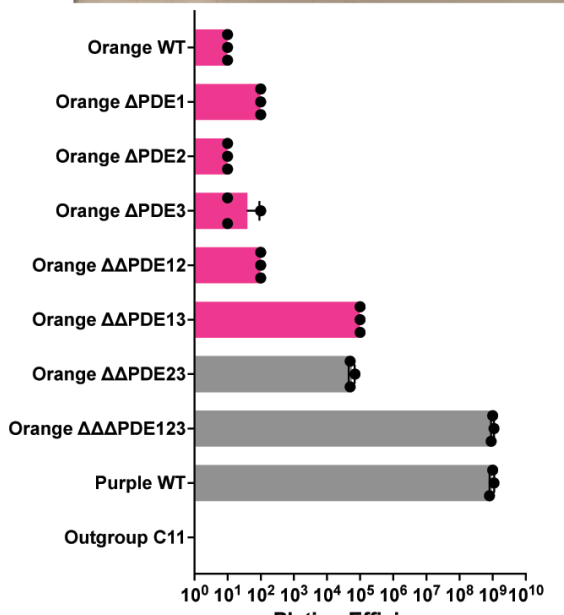

Plating Efficiency
$1: 10$ dilution series of representative

"purple" phage

propagated on $\triangle \triangle \triangle P D E 123$
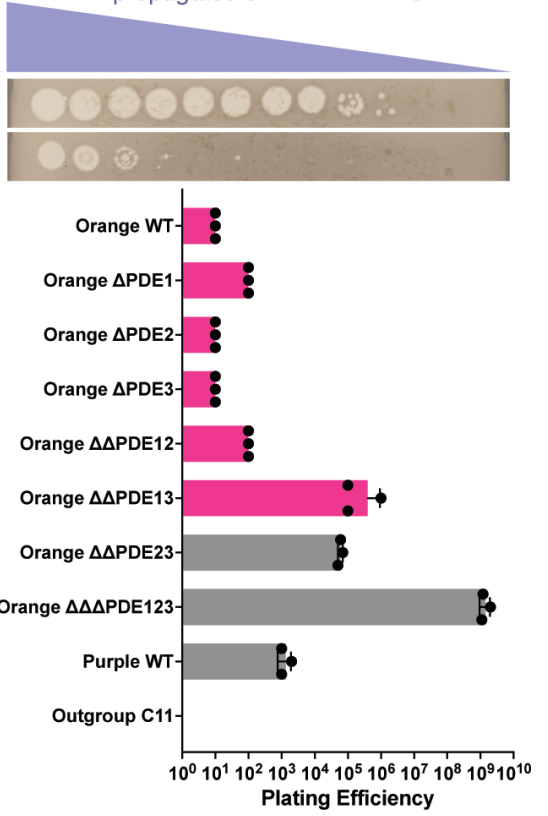

inhibition without plaque propagation

inhibition with plaque propagation

Fig. S14. Restriction modification phenotypic escape assayed using efficiency of plating. (A) WT "purple" phage tested on WT "purple" host compared to triple PDE knockout (left), and "purple" phage propagated on triple PDE knockout tested on both hosts (right). (B) All PDE knockouts tested against WT "purple" phage and "purple" phage propagated on triple PDE mutant. Pink bars indicate inhibition without plaque propagation and grey bars indicate killing with viable phage production. Triplicates shown as data points and error bars represent standard deviation. 
A

PDE1 defense characterization
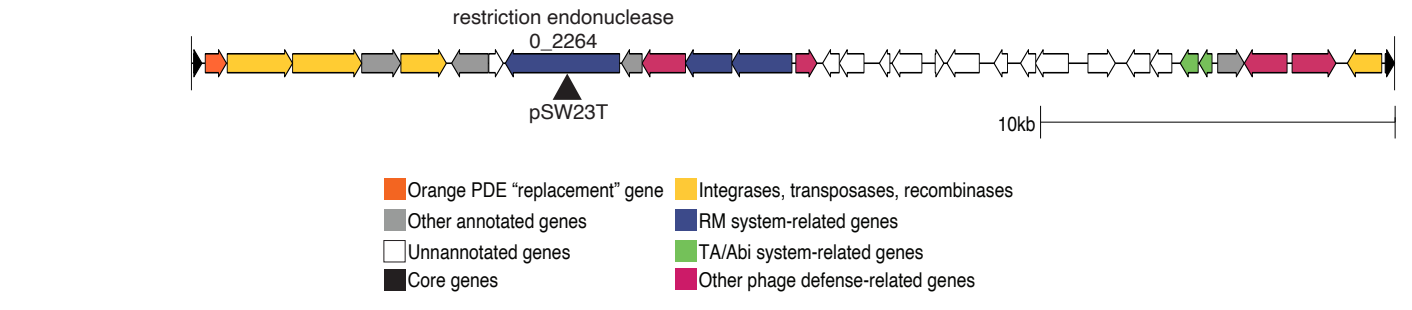

B

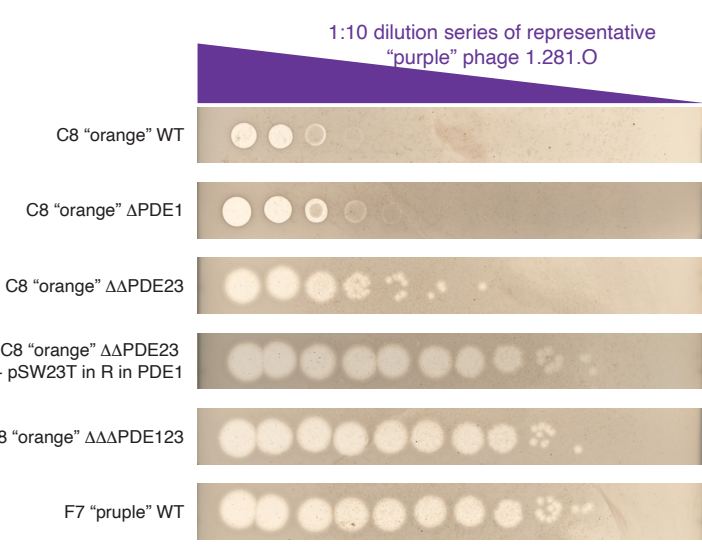

C
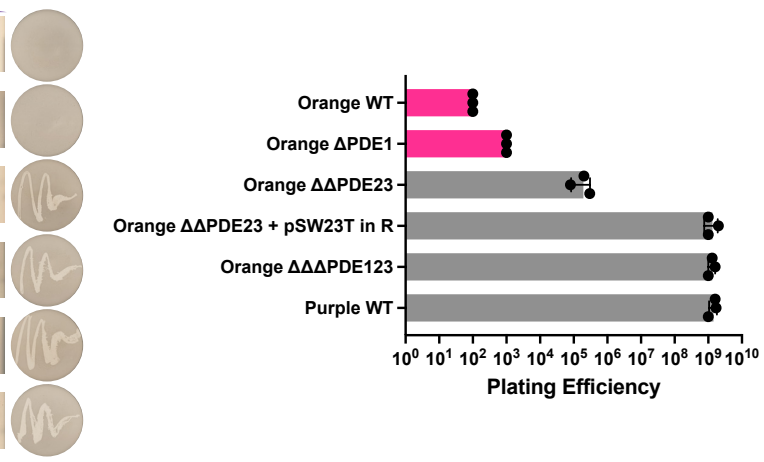

885

Fig. S15. Detailed genetic characterization of defense genes in PDE1. (A) Gene diagram depicting the inactivation of the restriction endonuclease (10N26155C8 ORF_0_2264) in the defense element double mutant $\triangle \triangle \mathrm{PDE} 23$ using a transposon. (B) Example of efficiency of 890 plating (EOP) assay showing that nearly full PDE deletions result in the same sensitivity to "purple" phages as inactivation of the restriction endonuclease (left). Re-streak to test for viable phage production (right). Plating done with representative "purple" phage 1.281.O. (C) Summary of replicate EOP assays. Pink bars indicate inhibition without plaque propagation and grey bars indicate killing with viable phage production. Triplicates shown as data points and error bars represent standard deviation. 
A

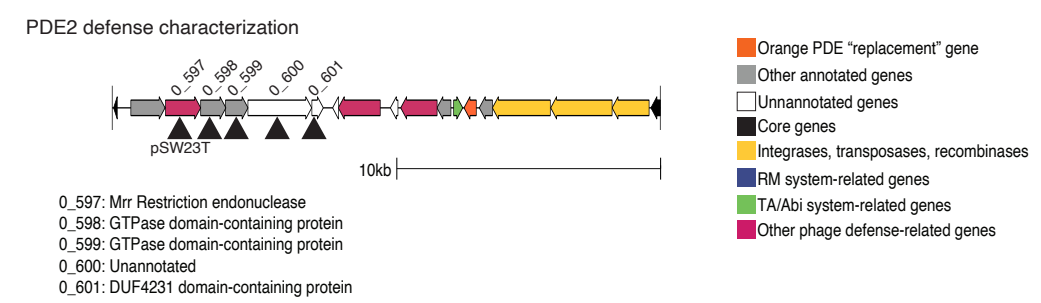

B

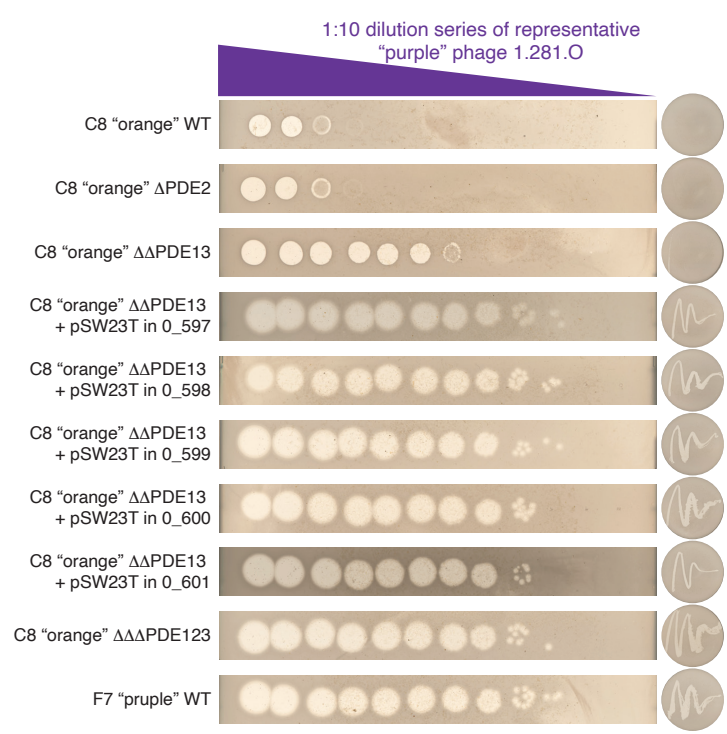

C

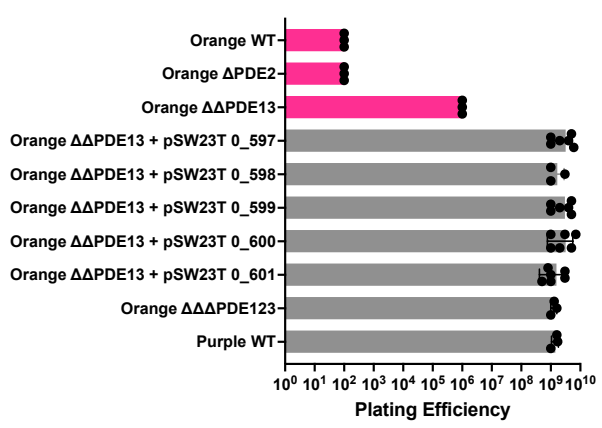

Fig. S16. Detailed genetic characterization of defense genes in PDE2. (A) Gene diagram depicting the inactivation of unannotated genes 10N26155C8 ORF_0_597, putative GTPase 10N26155C8 ORF_0_598, putative GTPase 10N26155C8 ORF_0_599, unannotated gene 10N26155C8 ORF_0_600, and unannotated gene 10N26155C8 ORF_0_601 with DUF4231 in the defense element double mutant $\Delta \Delta \mathrm{PDE} 13$ using transposons. (B) Example of efficiency of plating (EOP) assay showing that nearly full PDE deletions result in the same sensitivity to "purple" phages as inactivation of each gene in A (left). Re-streak to test for viable phage production (right). Plating done with representative "purple" phage 1.281.O. (C) Summary of replicate EOP assays. Pink bars indicate inhibition without plaque propagation and grey bars indicate killing with viable phage production. Triplicates shown as data points and error bars represent standard deviation. 

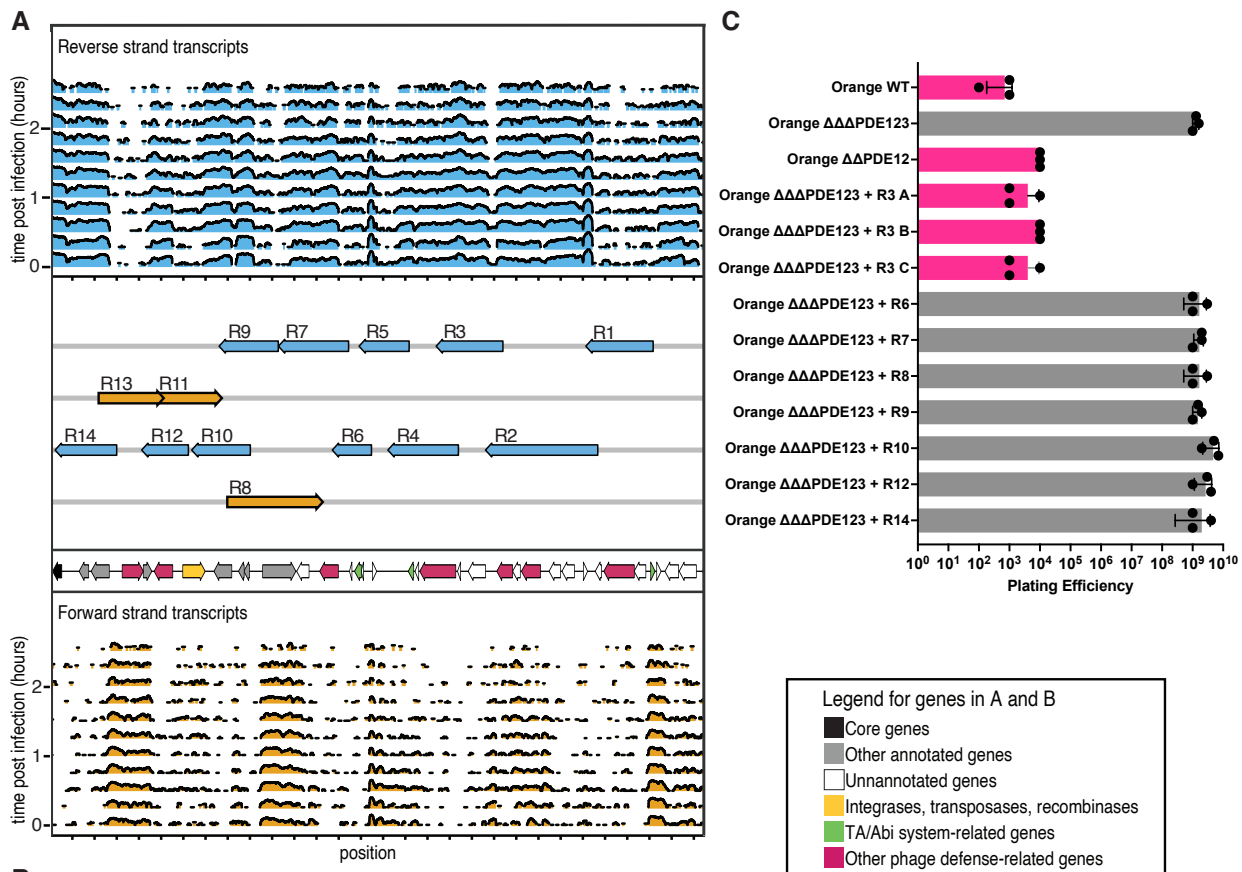

B
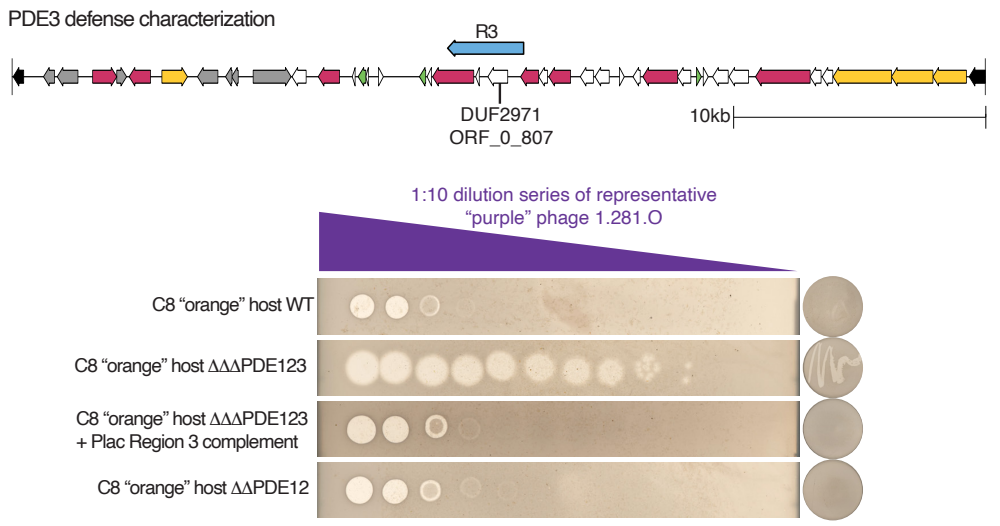

Fig. S17. Detailed genetic characterization of defense genes in PDE3. (A) Identification of cotranscribed regions (R1-R14) during a time course of infection of "orange" host 10N26155C8 with "purple" phage 1.281.O. (B, top) Gene diagram depicting complementation of R3 in the triple defense element mutant $\Delta \Delta \triangle \mathrm{PDE} 123$. R3 being the only region to restore resistance phenotype. (B, bottom) Example of efficiency of plating (EOP) assay showing that the presence of PDE3 results in the same resistance to "purple" phages as the complementation of R3 in $\Delta \triangle \triangle$ PDE123 (bottom, left). Re-streak to test for viable phage production (bottom, right). Plating done with representative "purple" phage 1.281.O. (C) EOP assay for all regions complemented in to triple PDE knockout. Pink bars indicate inhibition without plaque propagation and grey bars indicate killing with viable phage production. Triplicates shown as data points and error bars represent standard deviation. 

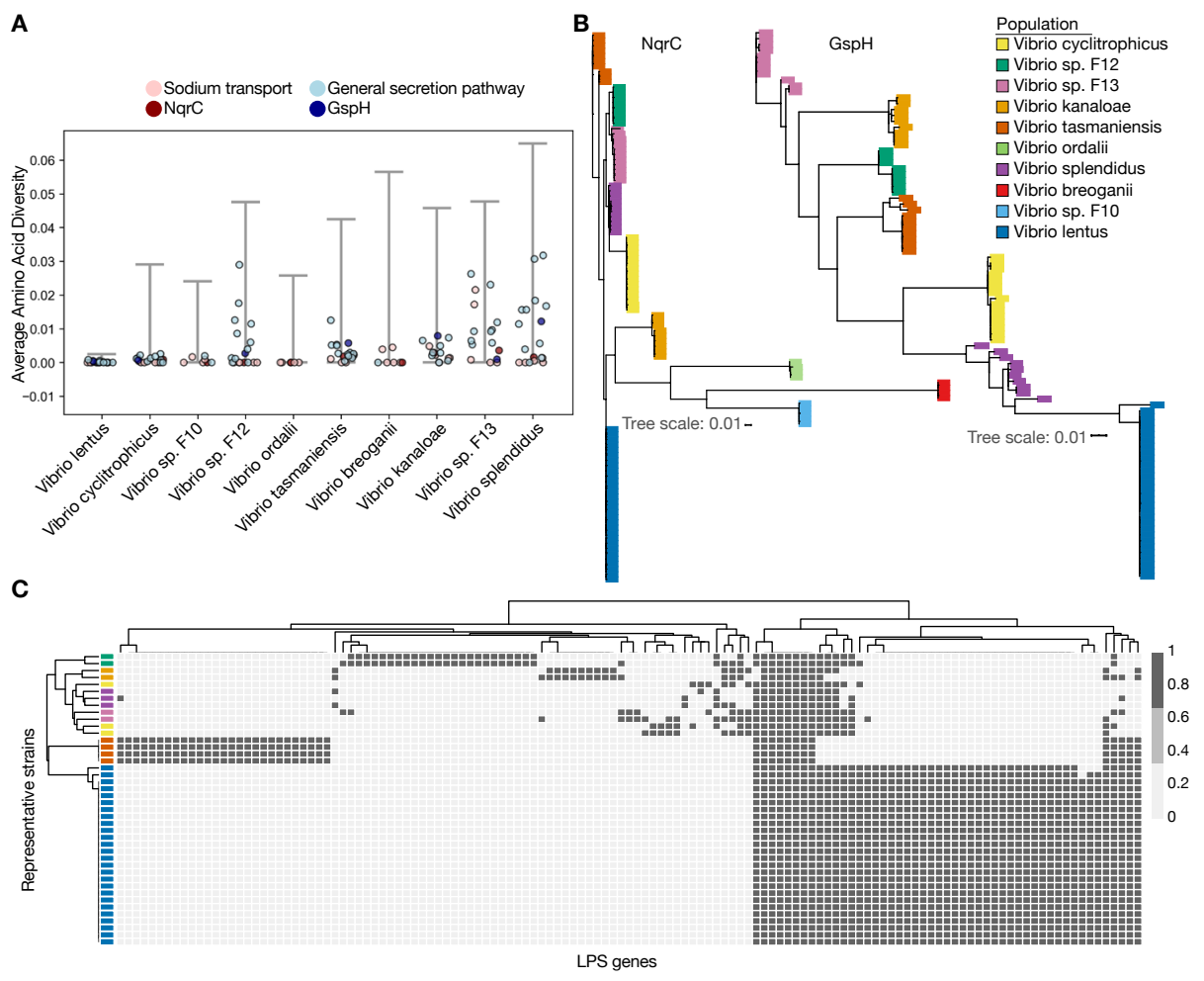

Fig. S18. Phage receptor diversity across diverse Vibrio species suggests positive selection at the population level. (A) Genes identified as receptors in $V$. lentus show much reduced diversity compared to core genes in 10 populations of vibrios. Populations are defined as co-existing members of species and represent gene flow units (37). Each point represents the average amino acid pairwise diversity for a specific receptor gene indicated by color. The grey bars represent the 2.5th and 97.5th percentile range of average amino acid pairwise diversity for all core genes within a given population. (B) Nucleotide trees of select receptor genes, sodium transporter $940 \quad(n q r C)$ and pseudopilin $(g s p H)$, show evidence of recent population-level sweeps since receptor alleles within each population are nearly identical. (C) Genes encoding the LPS show the same presence/absence patterns within populations indicating that they encode the same LPS variant, indicating receptor evolution is congruent with population evolution. Populations are defined here as gene flow clusters that also represent ecological units (37). The recognition of population 945 boundaries is key for interpretation of gene or allele frequency in the context of differential selective forces. 
A

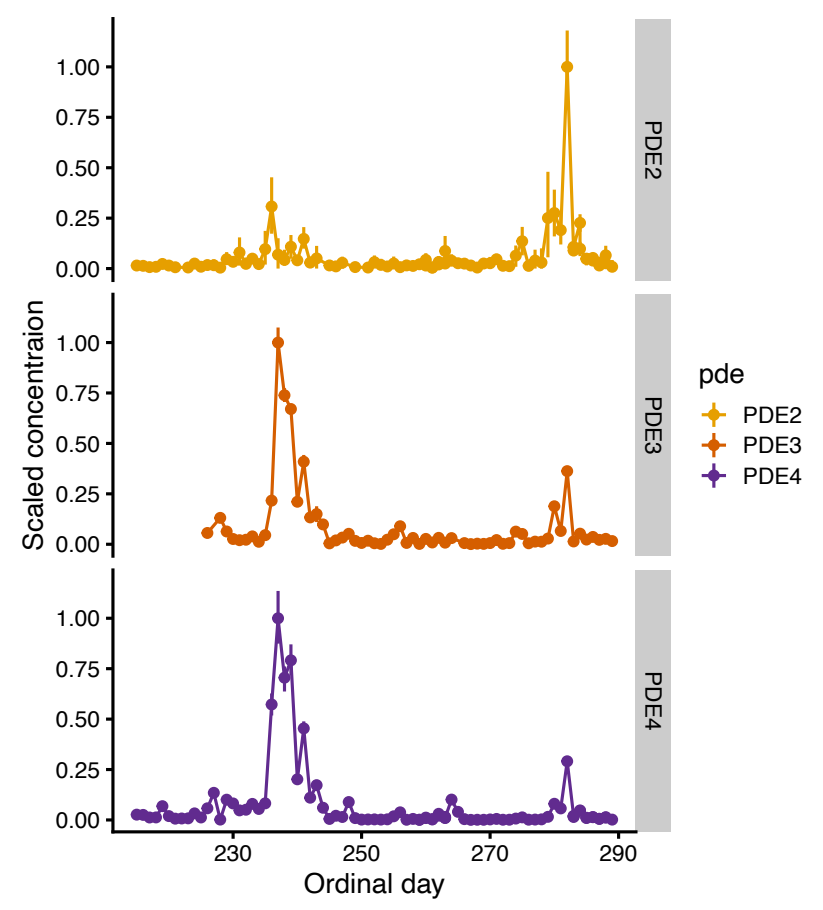

B

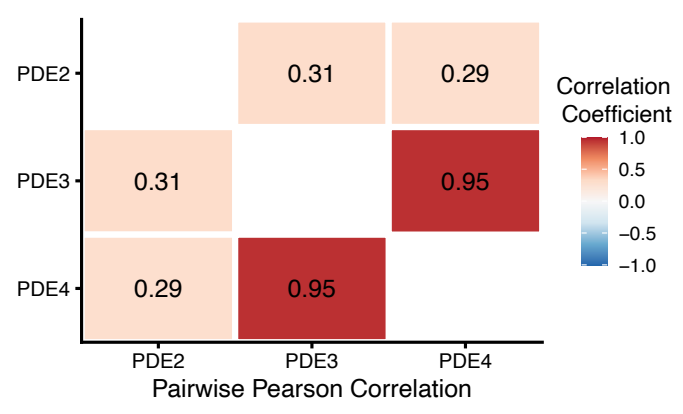

960

Fig. S19.Droplet digital PCR quantification of PDEs over time in the Nahant time series. (A) Relative abundance of PDE2 and PDE3 in "orange" clonal genomic background and PDE4 in "purple" clonal genomic background were quantified by designing an amplicon that spans the 965 insertion site of each PDE in its specific genomic background. Error bars represent Poisson 95\% confidence intervals. (B) Pairwise Pearson correlation of PDE relative abundances over the time series. 
A

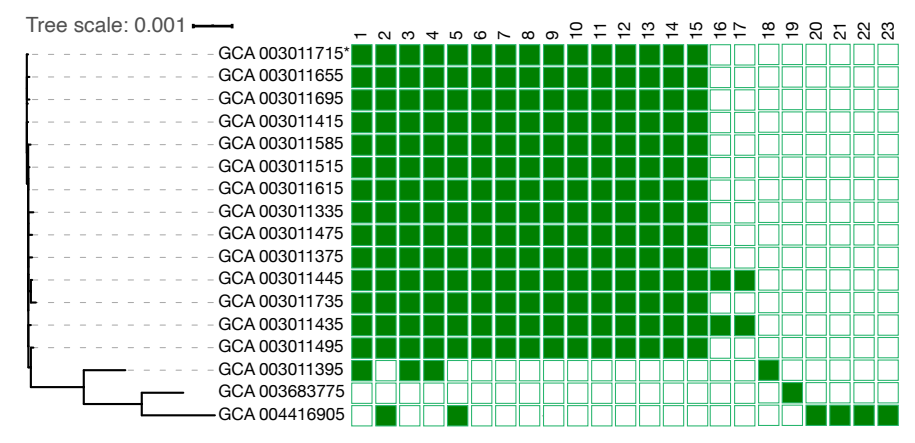

C $10 \mathrm{kpb}$
B

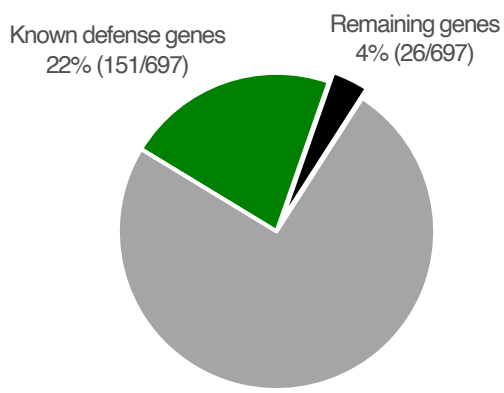

PDE genes minus known defense genes $74 \%(520 / 697)$

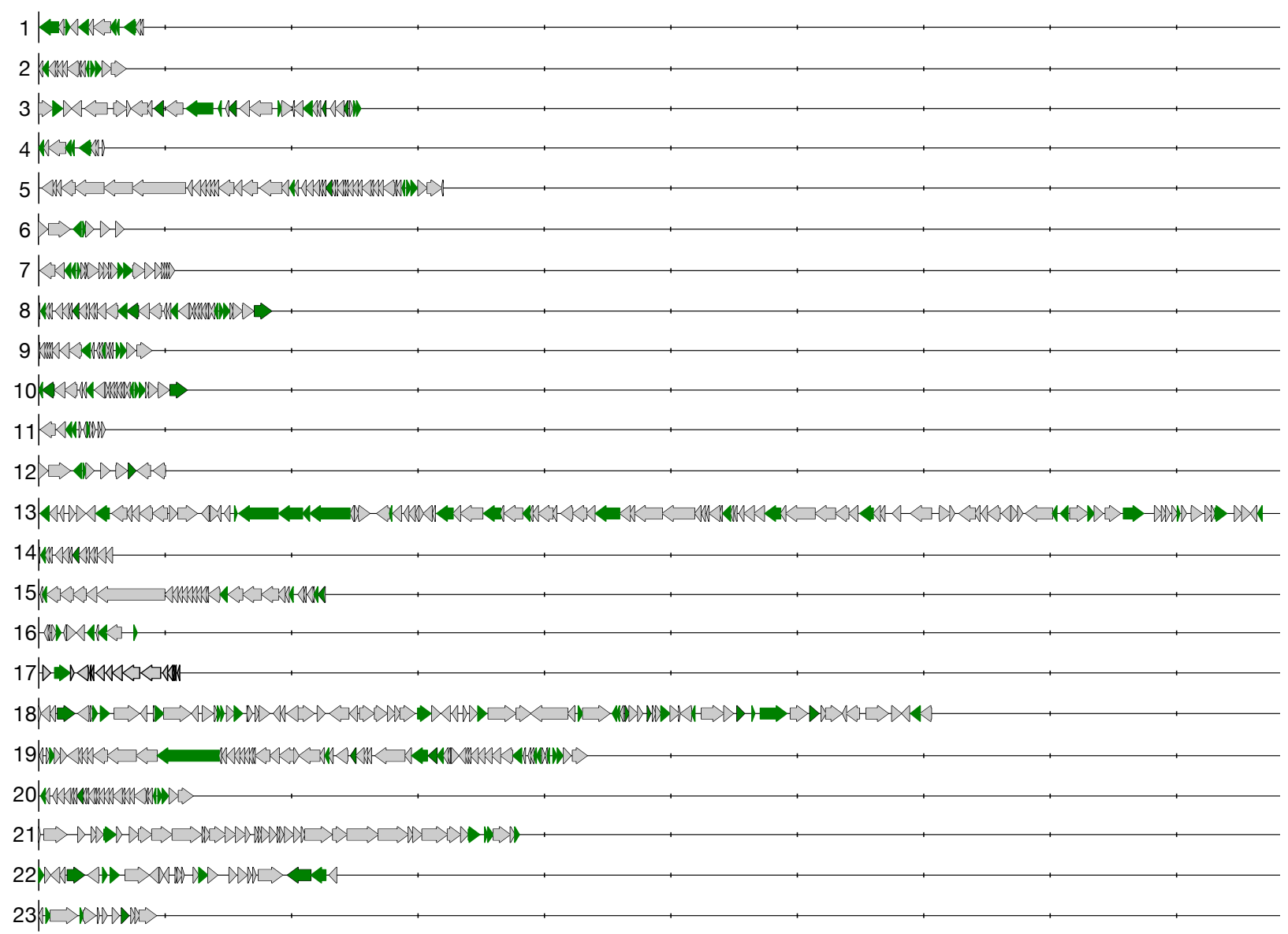

Fig. S20.Distribution of putative phage defense regions in closely related Listeria strains. (A) Core genome tree with presence/absence matrix of elements. (B) Flexible genome percentage breakdown of phage defense elements. (C) Accompanying gene diagrams of each phage defense element. In gene diagrams, hits to known defense genes are shown in green. See supplemental Data S4 for genes and annotations. 
A

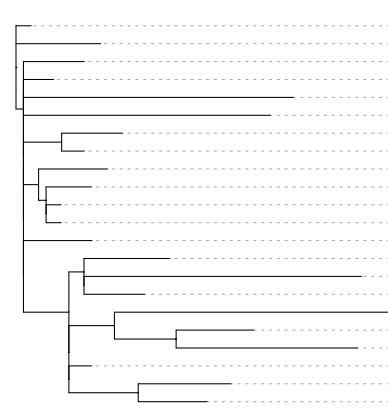

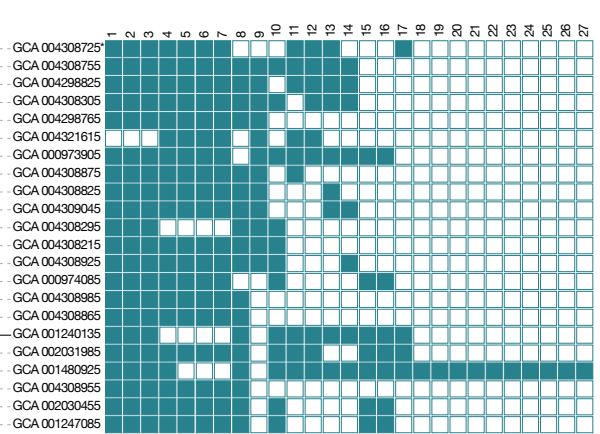

B

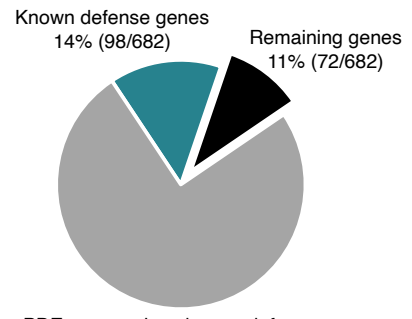

PDE genes minus known defense genes $75 \%(512 / 682)$

$\mathbf{C}^{\text {Treescale: } 0.01-1}$

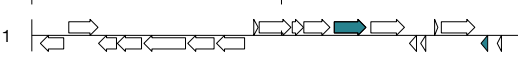

2 ए।

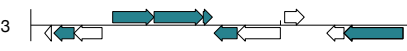

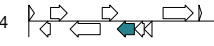

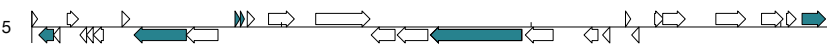

6 h

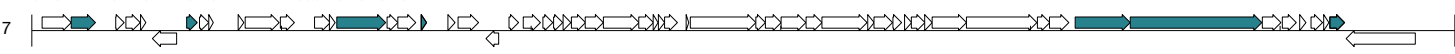

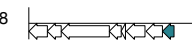

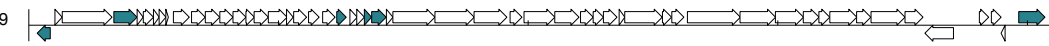

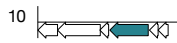

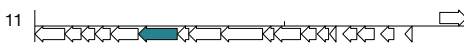

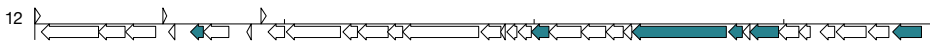

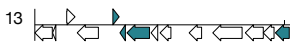

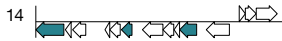

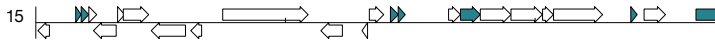

${ }^{16}$

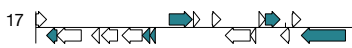

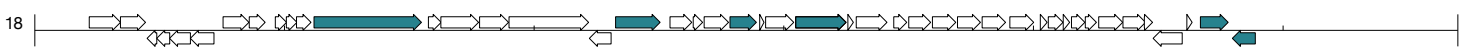

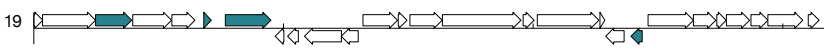

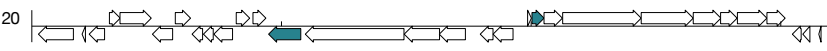

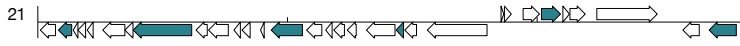

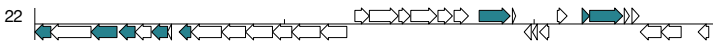

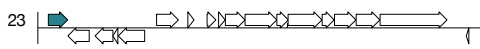

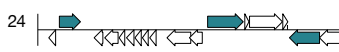

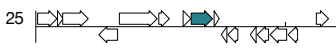

${ }^{26} \longmapsto$ CXKK

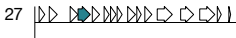

Fig. S21.Distribution of putative phage defense regions in closely related Salmonella strains. (A) Core genome tree with presence/absence matrix of elements. (B) Flexible genome percentage breakdown of phage defense elements. (C) Accompanying gene diagrams of each phage defense element. In gene diagrams, hits to known defense genes are shown in blue. See supplemental Data S5 for genes and annotations. 
A

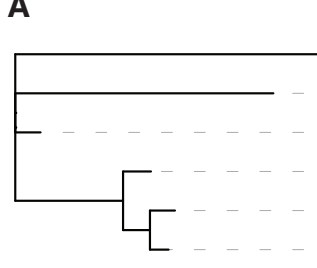

Tree scale: $0.1 \longmapsto$
B

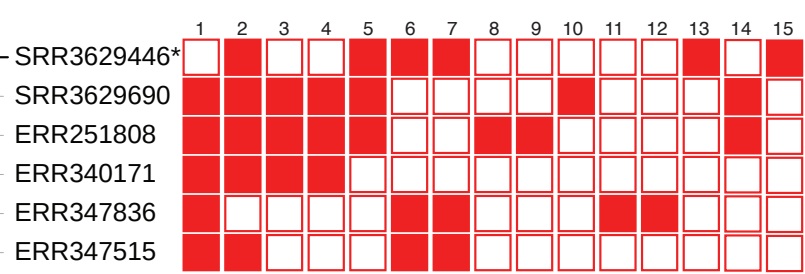

ERR347515

C

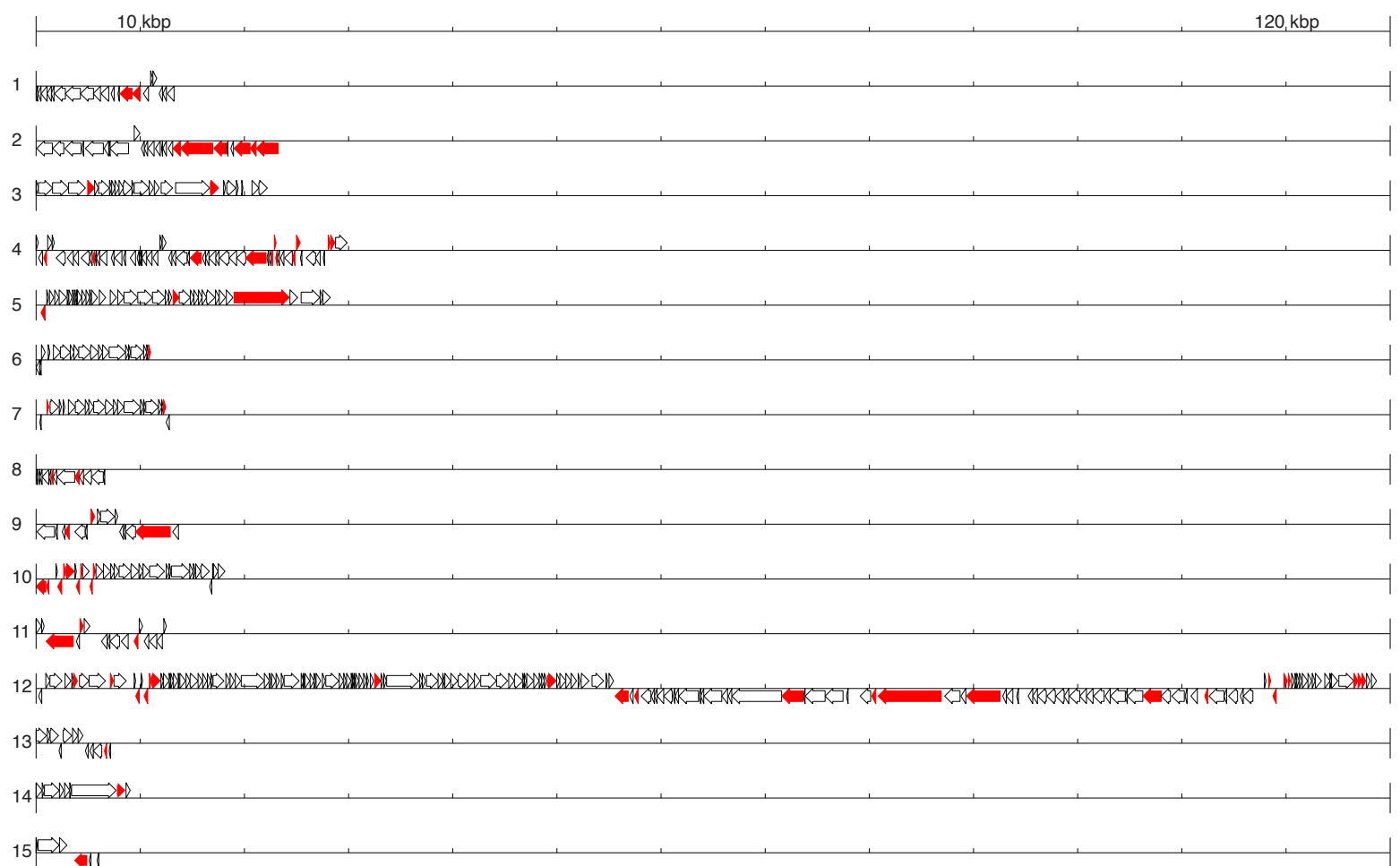

Fig. S22.Distribution of putative phage defense regions in closely related Clostridium strains. percentage breakdown of phage defense elements. (C) Accompanying gene diagrams of each phage defense element. In gene diagrams, hits to known defense genes are shown in red. See supplemental Data S6 for genes and annotations. 
Table S1.

Receptor identification by transposon mutagenesis. All mappings used 10N26155C8 as a reference.

\begin{tabular}{|c|c|c|c|}
\hline Phage & Gene ID & Annotation & Tn Hits \\
\hline 1.143 .0 & 10N26155C8_2_159 & General secretion pathway protein $\mathrm{H}(g s p H)$ & 81 \\
\hline 1.143 .0 & 10N26155C8_2_164 & General secretion pathway protein $\mathrm{C}(g s p C)$ & 9 \\
\hline 1.143 .0 & 10N26155C8_2_162 & General secretion pathway protein $\mathrm{E}(g s p E)$ & 1 \\
\hline 1.143 .0 & 10N26155C8_2_156 & General secretion pathway protein K (gspK) & 1 \\
\hline 1.281 .0 & 10N26155C8_2_94 & dTDP-4-dehydrorhamnose reductase (Ips3) & 58 \\
\hline 1.281 .0 & 10N26155C8_2_93 & dTDP-4-dehydrorhamnose 3,5-epimerase & 8 \\
\hline 1.281 .0 & 10N26155C8_2_96 & dTDP-glucose 4,6-dehydratase & 6 \\
\hline 1.281 .0 & 10N26155C8_0_669 & Phosphoglucomutase & 3 \\
\hline 1.281 .0 & 10N26155C8_0_485 & ABC-type multidrug transport system, ATPase and permease component & 1 \\
\hline 1.281 .0 & 10N26155C8_0_2482 & $\mathrm{Na}(+)$-translocating $\mathrm{NADH}$-quinone reductase subunit $\mathrm{B}(\mathrm{nqr} B)$ & 1 \\
\hline 1.281 .0 & 10N26155C8_0_2480 & $\mathrm{Na}(+)$-translocating $\mathrm{NADH}$-quinone reductase subunit $\mathrm{D}$ (nqrD) & 1 \\
\hline
\end{tabular}




\section{Table S2.}

Receptor identification by sequencing of spontaneous resistant mutants. Single nucleotide variants (SNVs) and deletions were identified using CLC work bench 9 workflow or though further manual identification using coverage analysis (CA). All mappings used 10N26155C8 as a reference.

\begin{tabular}{|c|c|c|c|c|c|c|c|}
\hline Phage & Replicate & Contig_Position & Type & Length & Gene & AA change & Annotation \\
\hline 1.119 .0 & 1 & 2_171942 & Deletion & 1 & ORF_2_160 & ORF_2_160:p.Lys7fs & $\begin{array}{c}\text { General secretion pathway protein } G \\
\qquad(g s p G)\end{array}$ \\
\hline 1.119 .0 & 2 & 2_173977 & SNV & 1 & ORF_2_162 & ORF_2_162:p.Lys267* & $\begin{array}{l}\text { General secretion pathway protein E } \\
(g s p E)\end{array}$ \\
\hline 1.119 .0 & 3 & 2_1671-167305 & $\begin{array}{c}\text { Deletion } \\
\text { via CA }\end{array}$ & 109 & ORF_2_154 & NA & $\begin{array}{c}\text { General secretion pathway protein M } \\
(g s p M)\end{array}$ \\
\hline 1.119 .0 & 4 & 2_167254 & SNV & 1 & ORF_2_154 & ORF_2_154:p.Trp63* & $\begin{array}{c}\text { General secretion pathway protein M } \\
(\text { (gspM) }\end{array}$ \\
\hline 1.119 .0 & 5 & 2_173221 & Deletion & 1 & ORF_2_161 & ORF_2_161:p.Gly18fs & $\begin{array}{c}\text { General secretion pathway protein } \mathrm{F} \\
(\mathrm{gsp} F)\end{array}$ \\
\hline 1.119 .0 & 6 & 2_1719-172018 & $\begin{array}{c}\text { Deletion } \\
\text { via CA }\end{array}$ & 83 & ORF_2_160 & NA & $\begin{array}{l}\text { General secretion pathway protein } G \\
(g s p G)\end{array}$ \\
\hline 1.119 .0 & 6 & $\begin{array}{l}2 \_166050- \\
166174\end{array}$ & $\begin{array}{c}\text { Deletion } \\
\text { via CA }\end{array}$ & 125 & ORF_2_153 & NA & $\begin{array}{c}\text { General secretion pathway protein } \mathrm{N} \\
(g s p N)\end{array}$ \\
\hline 1.281 .0 & 1 & 2_2804974 & SNV & 1 & ORF_0_2483 & ORF_0_2483:p.Ser302* & $\begin{array}{l}\mathrm{Na}(+) \text {-translocating } \mathrm{NADH}-\text { quinone } \\
\text { reductase subunit A (EC 1.6.5.-) (nqrA) }\end{array}$ \\
\hline 1.281 .0 & 2 & 2_68299 & Insertion & 1 & ORF_2_61 & ORF_2_61:p.Tyr187fs & Unannotated \\
\hline 1.281 .0 & 3 & 2_72111-72115 & $\begin{array}{c}\text { Deletion } \\
\text { via CA }\end{array}$ & 5 & NA & NA & $\begin{array}{c}\text { intergenic, upstream of } \\
\text { 10N26155C8_2_65 (Unannotated) }\end{array}$ \\
\hline 1.281 .0 & 3 & 2_72128-72134 & $\begin{array}{c}\text { Deletion } \\
\text { via CA }\end{array}$ & 7 & NA & NA & $\begin{array}{c}\text { intergenic, upstream of } \\
\text { 10N26155C8_2_65 (Unannotated) }\end{array}$ \\
\hline 1.281 .0 & 4 & 2_68299 & Insertion & 1 & ORF_2_61 & ORF_2_61:p.Tyr187fs & Unannotated \\
\hline 1.281 .0 & 5 & 2_108233 & SNV & 1 & ORF_2_96 & ORF_2_96:p.Gln332* & $\begin{array}{c}\text { dTDP-glucose 4,6-dehydratase (EC } \\
\text { 4.2.1.46) }\end{array}$ \\
\hline 1.281 .0 & 6 & 2_68845-68906 & $\begin{array}{c}\text { Deletion } \\
\text { via CA }\end{array}$ & 62 & ORF_2_61\&62 & NA & $\begin{array}{l}\text { end of } 10 \mathrm{~N} 26155 \mathrm{C} 8 \_2 \_61 \text { and } \\
\text { beginning of 10N26155C8_2_62 }\end{array}$ \\
\hline 1.281 .0 & 7 & 2_107805 & SNV & 1 & ORF_2_95 & ORF_2_95:p.Asn109Tyr & $\begin{array}{c}\text { Glucose-1-phosphate } \\
\text { thymidylyltransferase (EC 2.7.7.24) }\end{array}$ \\
\hline
\end{tabular}


Table S3.

Core genome SNPs separating "purple" and "orange" clones (matching Fig. S8). Strain $10 \mathrm{~N} 28654 \mathrm{~F} 7$ was used as the reference.

\begin{tabular}{|c|c|c|c|c|c|}
\hline $\begin{array}{c}\text { SNP } \\
\#\end{array}$ & $\begin{array}{c}\text { Nucleotide } \\
\text { change } \\
\text { P-->O }\end{array}$ & $\begin{array}{c}\text { ORF } \\
\text { in 10N28654F7 }\end{array}$ & Annotation & $\begin{array}{c}\text { AA change } \\
\text { P-->O }\end{array}$ & Notes \\
\hline 1 & G-->T & ORF_0_1115 & $\begin{array}{l}\text { Cytochrome O ubiquinol } \\
\text { oxidase subunit III }\end{array}$ & Val-->Phe & \\
\hline 2 & C-->T & ORF_0_1604 & Unannotated & NA & $\begin{array}{l}\text { Asp-->Asp; Near end of a } \\
\text { PDE }\end{array}$ \\
\hline 3 & C-->T & ORF_1_1456 & $\begin{array}{l}\text { Pesticidial crystal protein } \\
\text { cry6Aa }\end{array}$ & GIn-->Stop & \\
\hline 4 & $G-->A$ & ORF_1_917 & $\begin{array}{c}\text { Aerobic cobaltochelatase CobS } \\
\text { subunit }\end{array}$ & Ala-->Val & \\
\hline 5 & G-->A & ORF_1_912 & $\begin{array}{c}\text { TRAP-type C4-dicarboxylate } \\
\text { transport system, periplasmic } \\
\text { component }\end{array}$ & NA & Gly-->Gly \\
\hline 6 & $A-->G$ & $\begin{array}{c}\mathrm{ORF}_{366}^{1} 365- \\
\end{array}$ & Unannotated & Stop-->GIn & \\
\hline 7 & $C-->T$ & ORF_0_3133 & $\begin{array}{c}\text { Phosphoenolpyruvate } \\
\text { carboxylase }\end{array}$ & NA & Gly-->Gly \\
\hline 8 & $\mathrm{C}-->\mathrm{T}$ & ORF_0_1978 & IcmF-related protein & Ala-->Val & \\
\hline 9 & $A-->G$ & ORF_1_87 & Unannotated & Thr-->Ala & \\
\hline 10 & $A-->G$ & intergenic & NA & NA & $\begin{array}{l}\text { upstream of ORF_1_86: } \\
\text { putative orphan protein; } \\
\text { putative membrane protein }\end{array}$ \\
\hline 11 & $C-->G$ & ORF_1_687 & $\begin{array}{l}\text { RND multidrug efflux } \\
\text { transporter; Acriflavin } \\
\text { resistance protein }\end{array}$ & Gln-->Glu & \\
\hline 12 & $G-->A$ & ORF_0_1847 & $\begin{array}{c}\text { Formate dehydrogenase }-\mathrm{O}, \\
\text { gamma subunit }\end{array}$ & Met-->lle & \\
\hline 13 & $C-->A$ & intergenic & NA & NA & $\begin{array}{c}\text { upstream of } \\
\text { ORF_0_138:tRNA uridine } \\
5- \\
\text { carboxymethylaminomethyl } \\
\text { modification enzyme GidA }\end{array}$ \\
\hline 14 & G-->A & ORF_1_1585 & Probable MFS transporter & NA & Ala-->Ala \\
\hline
\end{tabular}


Table S4.

Predicted motifs of RM systems on PDEs and corresponding methylome data.

\begin{tabular}{|c|c|c|}
\hline RM PDE & PDE 1 & \\
\hline \multirow[t]{11}{*}{ Predicted Motif } & TCA*BN(4)RTRTC & \\
\hline & Host/Phage & Fraction Methylated \\
\hline & $10 \mathrm{~N} 26155 \mathrm{C} 8$ & $599 / 600$ \\
\hline & 1.119 .0 & $1 / 1$ \\
\hline & 1.127 .0 & $1 / 1$ \\
\hline & 1.143 .0 & $1 / 1$ \\
\hline & 1.231 .0 & $1 / 1$ \\
\hline & 10N28654F7 & $0 / 599$ \\
\hline & $1.283 . \mathrm{A}$ & $0 / 7$ \\
\hline & 1.281 .0 & $0 / 8$ \\
\hline & 1.196 .0 & $0 / 8$ \\
\hline RM PDE & PDE 4 & \\
\hline \multirow[t]{11}{*}{ Predicted Motif } & $\mathrm{CCA}^{*} \mathrm{GN}(6) \mathrm{TAA}$ & \\
\hline & Host/Phage & Fraction Methylated \\
\hline & 10N26155C8 & $0 / 646$ \\
\hline & 1.119 .0 & $0 / 3$ \\
\hline & 1.127 .0 & $0 / 4$ \\
\hline & 1.143 .0 & $0 / 3$ \\
\hline & 1.231 .0 & $0 / 3$ \\
\hline & 10N28654F7 & $635 / 638$ \\
\hline & 1.283.A & $5 / 5$ \\
\hline & 1.281 .0 & $4 / 4$ \\
\hline & 1.196 .0 & $4 / 4$ \\
\hline RM PDE & PDE 5 & \\
\hline \multirow[t]{11}{*}{ Predicted Motif } & $\mathrm{GA}^{*} \mathrm{GN}(6) \mathrm{GGC}$ & \\
\hline & Host/Phage & Fraction Methylated \\
\hline & 10N26155C8 & 0/1795 \\
\hline & 1.119 .0 & $0 / 17$ \\
\hline & 1.127 .0 & $0 / 15$ \\
\hline & 1.143 .0 & $0 / 17$ \\
\hline & 1.231 .0 & $0 / 17$ \\
\hline & 10N28654F7 & $1770 / 1779$ \\
\hline & 1.283.A & $8 / 8$ \\
\hline & 1.281 .0 & $10 / 10$ \\
\hline & 1.196 .0 & $10 / 10$ \\
\hline
\end{tabular}


